\title{
EFFECT OF ADAPTIVE TABS ON DRAG OF A SQUARE-BASE BLUFF BODY
}

\author{
A Thesis \\ presented to \\ the Faculty of California Polytechnic State University, \\ San Luis Obispo
}

\author{
In Partial Fulfillment \\ of the Requirements for the Degree \\ Master of Science in Aerospace Engineering
}

by

Brian William Barker

August 2014 
(C) 2014

Brian William Barker

ALL RIGHTS RESERVED 
TITLE:

Effect of Adaptive Tabs on Drag of a SquareBase Bluff Body

AUTHOR:

Brian William Barker

DATE SUBMITTED:

August 2014

COMMITTEE CHAIR: Jin Tso, Ph.D.

Professor of Aerospace Engineering

COMMITTEE MEMBER: Dianne DeTurris, Ph.D.

Professor of Aerospace Engineering

COMMITTEE MEMBER: $\quad$ Faysal Kolkailah, Ph.D.

Professor of Aerospace Engineering

COMMITTEE MEMBER: Russell Westphal, Ph.D.

Professor of Mechanical Engineering 


\section{ABSTRACT \\ Effect of Adaptive Tabs on Drag of a Square-Base Bluff Body Brian William Barker}

This thesis involves the experimental wind tunnel testing of a $0.127 \mathrm{~m}$ by $0.127 \mathrm{~m}$ square-base bluff body to test the effectiveness of trailing edge tabulations to reduce drag in the Cal Poly $0.912 \mathrm{~m}$ by $1.219 \mathrm{~m}$ low-speed wind tunnel. To accomplish this, the boundary layer was first measured on the trailing edge of the model for the three speeds at 10,20 , and $30 \mathrm{~m} / \mathrm{s}$, with $\mathrm{Re}=8.3 \mathrm{e} 4,1.6 \mathrm{e} 5$ and $2.5 \mathrm{e} 5$ respectively, without the tabs. Three different tests were performed to determine the effectiveness of the tabs. These tests included base pressure measurements, total drag force measurements and hotwire velocity fluctuation measurements. These tests were repeated with tabs on the model's trailing edge at the three different tab heights and without tabs at all three test speeds.

The base pressure measurements showed a decrease in average base pressure with the addition of tabs which signifies an increase in drag. The total drag measurements confirmed this by showing that the overall force increases with the addition of the tabs. The hotwire tests further confirm this by showing that the vortex is present for every configuration tested.

This thesis showed that the addition of tabs was unsuccessful in reducing the effects of the vortex shedding for a square-base bluff body. The addition of low, medium, and high tabs to the square base of the bluff body all showed an increase in vortex strength and overall drag. Further study is required to determine if drag savings are feasible for tabs all around the square base of the bluff body and at different locations. 


\section{ACKNOWLEDGMENTS}

Through this long process of designing, testing and defending I have many thanks to give. First I would like to thank Dr. Tso for taking me on as a graduate student and guiding me through the experiment. I would then like to thank Cody Thompson for his help building the model and maintaining the wind tunnel. I would also like to thank my amazing family and friends for all of their support and encouragement through this long and difficult process. 


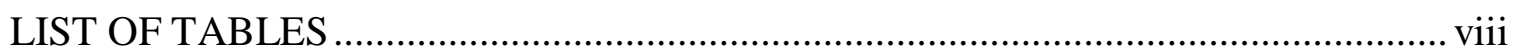

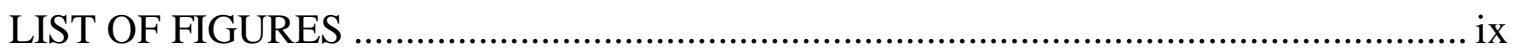

\section{CHAPTER}

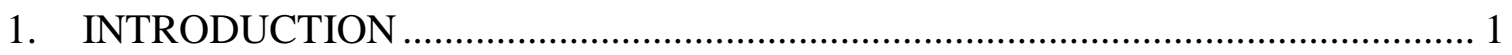

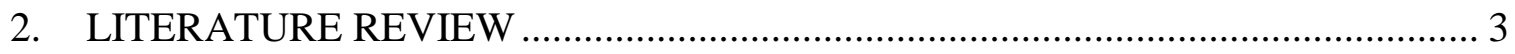

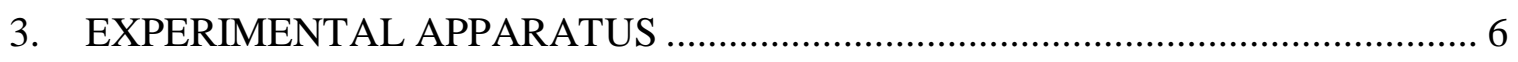

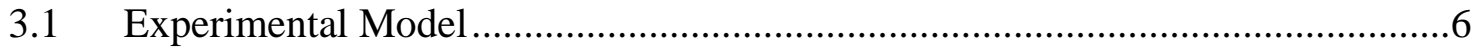

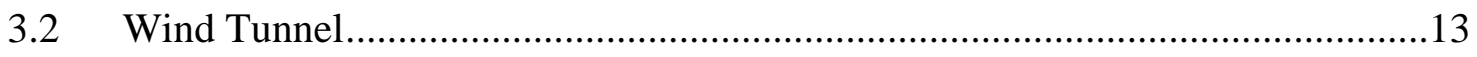

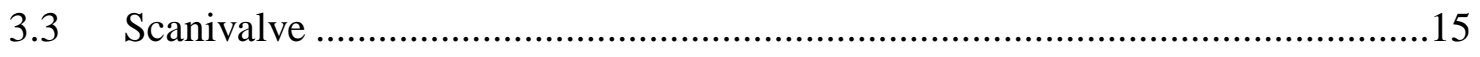

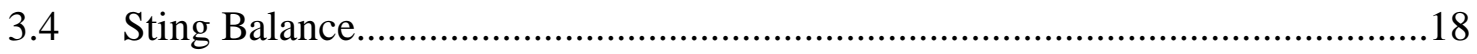

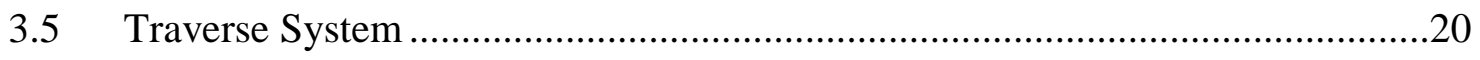

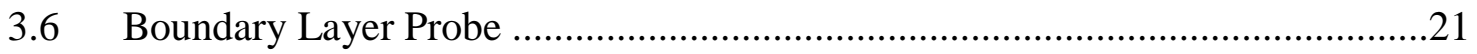

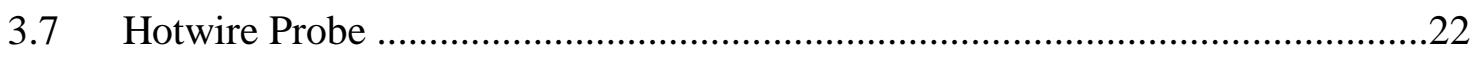

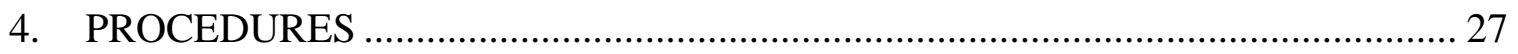

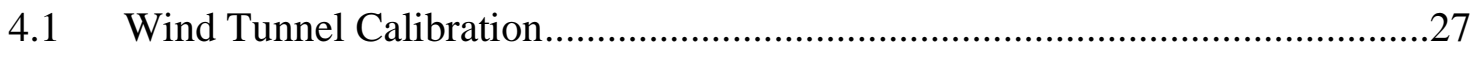

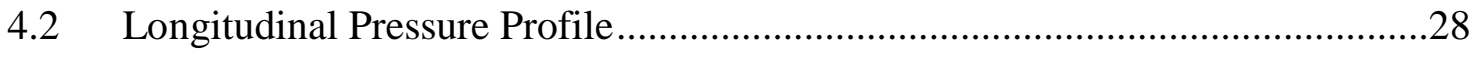

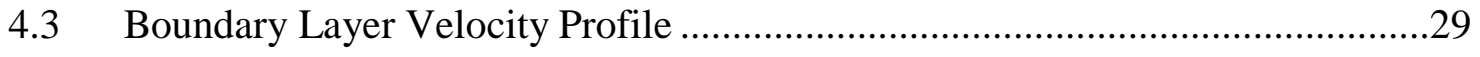

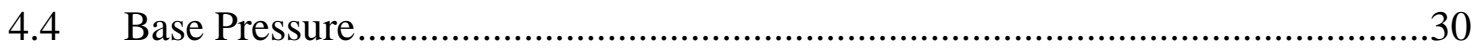

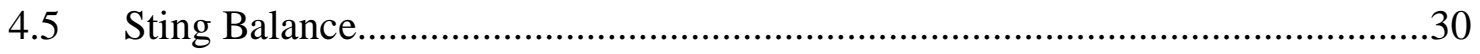

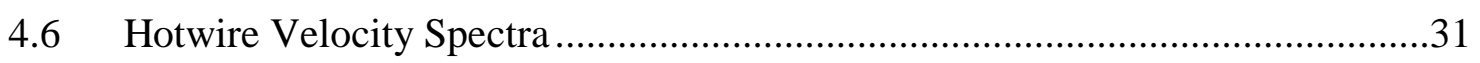

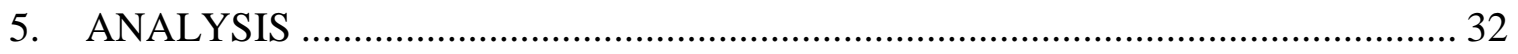

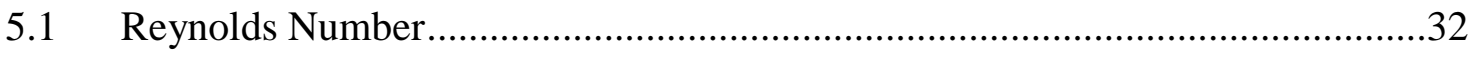




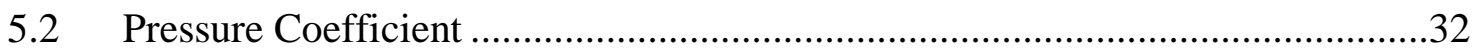

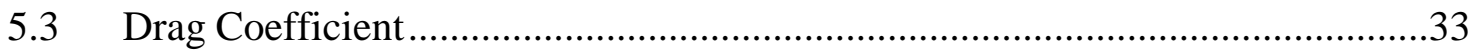

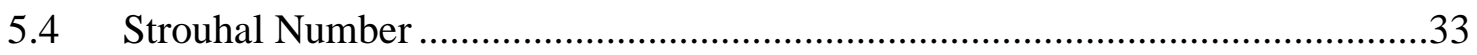

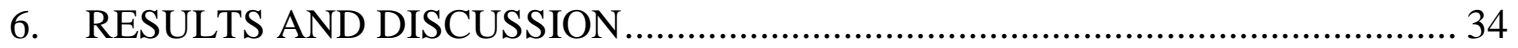

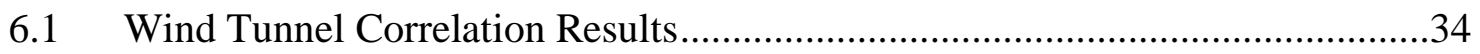

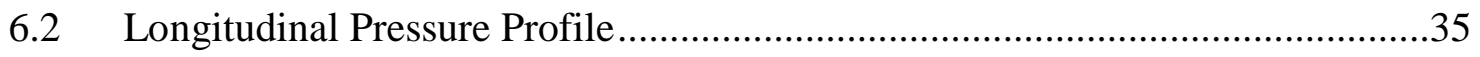

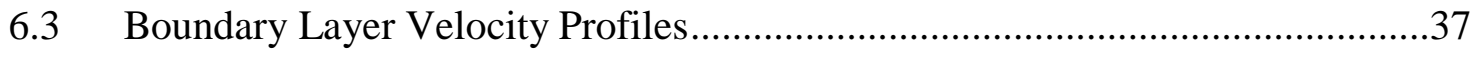

6.4 Base Pressure Coefficients ........................................................................41

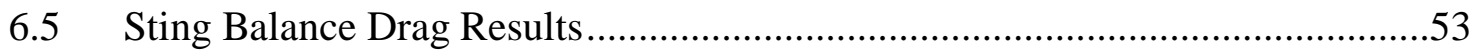

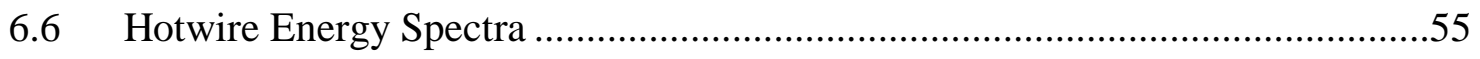

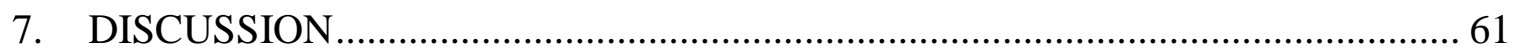

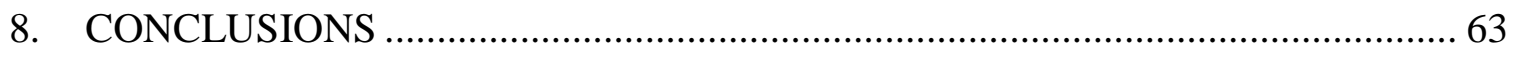

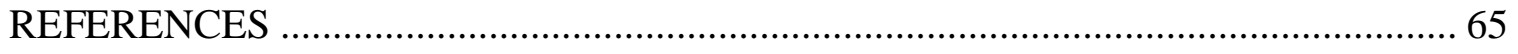

APPENDICES

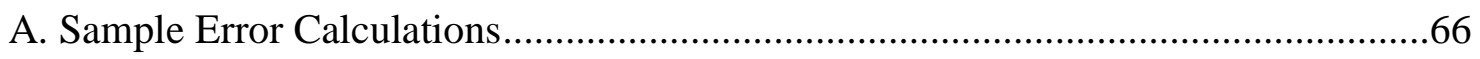

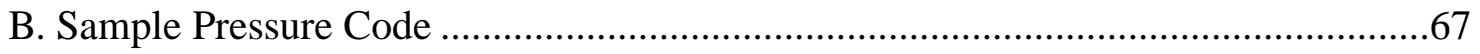

C. Sample Boundary Layer Code ..................................................................69 


\section{LIST OF TABLES}

Table Page

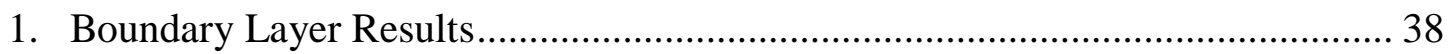

2. Changes in average $\mathrm{C}_{\mathrm{Pb}}$ Magnitude Compared to No Tab Configuration. ........... 47

3. Change in $C_{D}$ with Tabs Compared to No Tab Configuration. .......................... 55 


\section{LIST OF FIGURES}

Figure

Fig. 1. Experimental model mounted in wind tunnel.............................................. 6

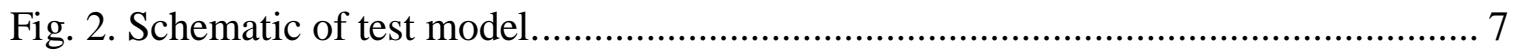

Fig. 3. Removable rear plates and rear plate on model in wind tunnel........................... 8

Fig. 4. Rear plate schematic. Tab heights are $0.0059 \mathrm{~m}, 0.0043 \mathrm{~m}$, and $0.0038 \mathrm{~m}$

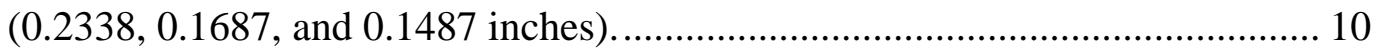

Fig. 5. Side and back plate pressure port distribution schematic............................... 11

Fig. 6. Hollow strut mount attached to experimental model........................................ 13

Fig. 7. Cal Poly wind tunnel laboratory. On the left there is a removable test

section. Next to the removed section is the inlet followed by the attached test

section and then the exhaust nozzle........................................................... 14

Fig. 8. Adapter plate (left), ZOC33 Scanivalve (right), and vacuum gun (top)............... 16

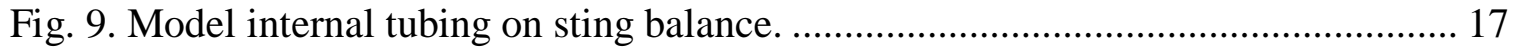

Fig. 10. Manometer used to calibrate the scanivalve.............................................. 17

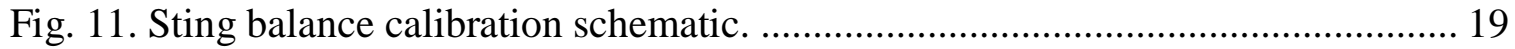

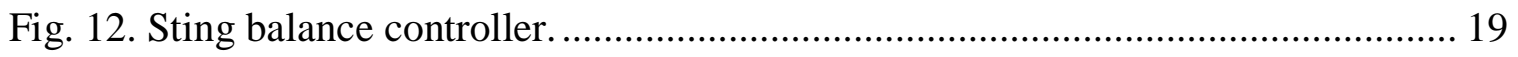

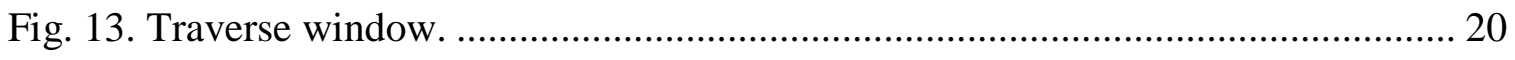

Fig. 14. Traverse mounted to side of wind tunnel. ............................................... 21

Fig. 15. Boundary layer probe at the trailing edge of the model................................. 22

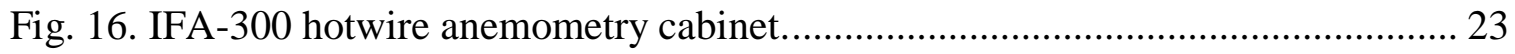

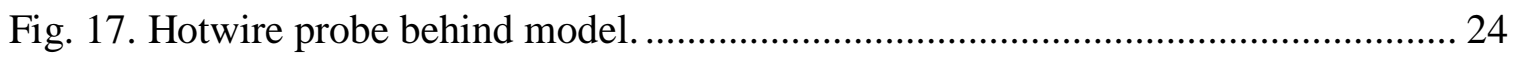

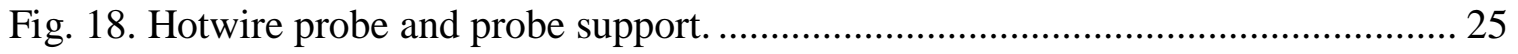

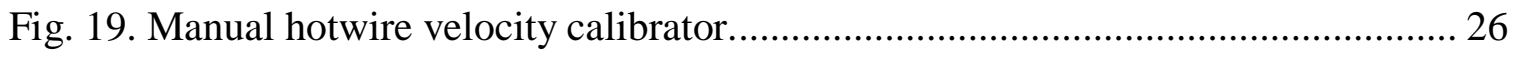


Fig. 20. Wind tunnel Variable Frequency Drive controller (right) and dynamic

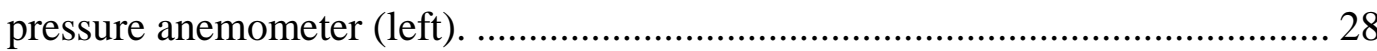

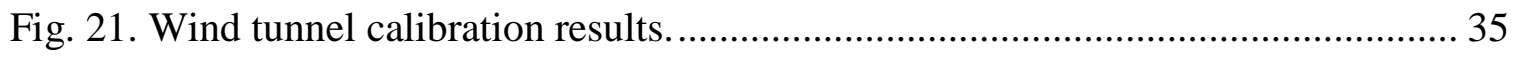

Fig. 22. This experiment's pressure coefficient profile results.................................... 36

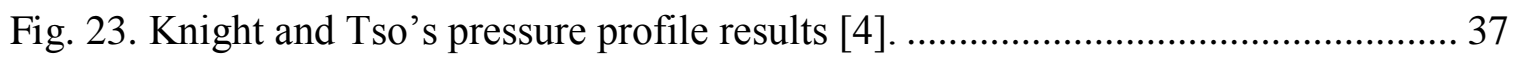

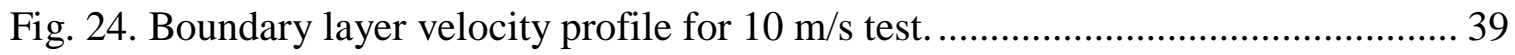

Fig. 25. Boundary layer velocity profile for $20 \mathrm{~m} / \mathrm{s}$ test......................................... 40

Fig. 26. Boundary Layer velocity profile for $30 \mathrm{~m} / \mathrm{s}$ testing. .................................. 41

Fig. 27. Park et al. data [3]. Solid square was optimally controlled flow with a pair of tabs. Solid circle data was uncontrolled flow. Open circle data was Bearman's uncontrolled flow. Solid triangle data was a two dimensional

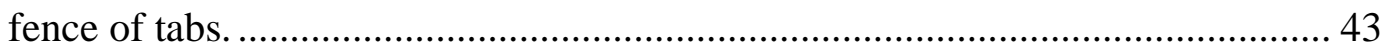

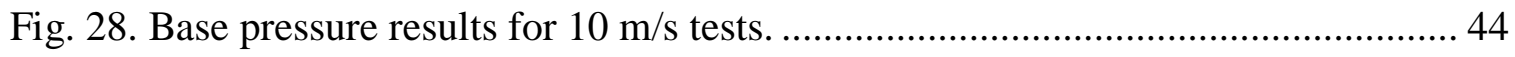

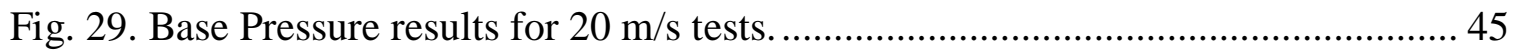

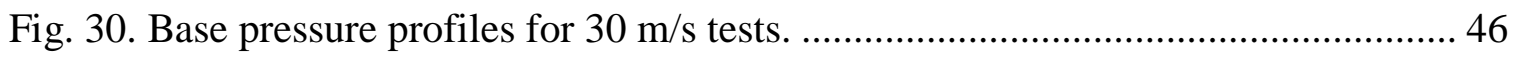

Fig. 31. Normal base $\mathrm{C}_{\mathrm{Pb}}$ distribution for each tab height with varying speeds............. 48

Fig. 32. Spanwise base pressure results for $10 \mathrm{~m} / \mathrm{s}$ tests. ........................................ 49

Fig. 33. Spanwise base pressure results for $20 \mathrm{~m} / \mathrm{s}$ tests. ..................................... 50

Fig. 34. Spanwise base pressure results for $30 \mathrm{~m} / \mathrm{s}$ tests. ...................................... 51

Fig. 35. Spanwise base $C_{p b}$ distribution for each tab height with varying speeds. ......... 52

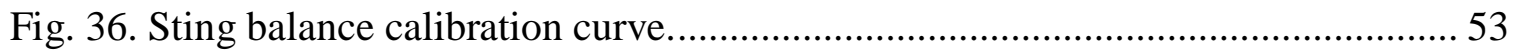

Fig. 37. Coefficient of drag results for all four tab configurations at all three speeds. 
Fig. 38. Calibration curve for the hotwire probe.

Fig. 39. Energy spectra results for $10 \mathrm{~m} / \mathrm{s}$ speed for all four tab configurations. ............ 57

Fig. 40. Energy spectra results for $20 \mathrm{~m} / \mathrm{s}$ speed for all four tab configurations. ............ 58

Fig. 41. Energy spectra results for $30 \mathrm{~m} / \mathrm{s}$ speed for all four tab configurations............. 59

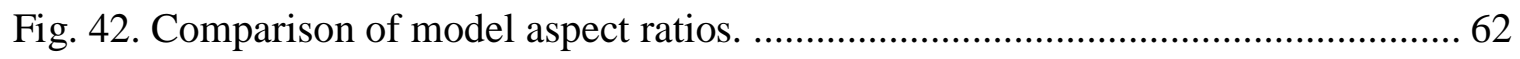

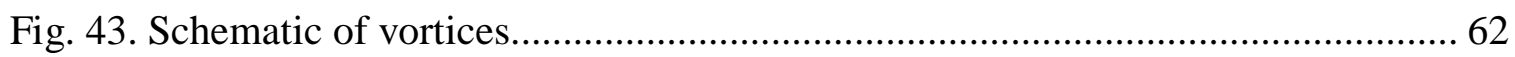




\section{NOMENCLATURE}

Greek

$\delta$

$\varepsilon_{\mathrm{sb}}$

$\rho$

$\theta$

$\lambda$

Alpha Numeric

2D

$3 \mathrm{D}$

$\mathrm{C}_{\mathrm{D}}$

$\mathrm{C}_{\mathrm{P}}$

$\mathrm{C}_{\mathrm{Pb}}$

f

FFT

$\mathrm{h}$

$\mathrm{K}_{\varepsilon}$

$l_{\mathrm{y}}$

$l_{\mathrm{z}}$

$\mathrm{p}_{\text {tot }}$

$\mathrm{p}_{\infty}$

$\mathrm{q}_{\infty}$

$\mathrm{Re}$

$\mathrm{St}$

$\mathrm{U}_{\infty}$

$\mathrm{V}$
Boundary layer height

Solid blockage coefficient

Density

Momentum thickness

Spacing between tabs

Two dimensional

Three dimensional

Coefficient of drag, $\frac{D}{q_{\infty} S}$.

Coefficient of pressure, $\frac{p-p_{\infty}}{q_{\infty}}$.

Base coefficient of pressure, $\frac{p_{b}-p_{\infty}}{q_{\infty}}$

Frequency

Fast Fourier transform

Height of the model

Blockage coefficient

Height of tabs

Width of tabs

Total pressure

Free stream pressure

Free stream dynamic pressure

Reynolds number, $\frac{U_{\infty} h}{v}$

Strouhal number, $\frac{f h}{U_{\infty}}$

Free stream velocity

Voltage 


$\begin{array}{ll}\mathrm{u}, \mathrm{v}, \mathrm{w} & \begin{array}{l}\text { Streamwise, normal and spanwise velocity } \\ \mathrm{x}, \mathrm{y}, \mathrm{z}\end{array} \\ \text { Streamwise, normal and spanwise coordinates } \\ \begin{array}{l}\text { Superscripts and } \\ \text { Subscripts }\end{array} \\ \mathrm{D} \\ \mathrm{P} & \text { Drag } \\ \mathrm{Pb} & \text { Pressure } \\ \mathrm{tot} & \text { Base pressure } \\ \mathrm{u}, \mathrm{v}, \mathrm{w} & \text { Total (pressure) } \\ \mathrm{x}, \mathrm{y}, \mathrm{z} & \text { Streamwise, normal and spanwise velocity } \\ \infty & \text { Streamwise, normal and spanwise coordinates } \\ & \text { Free stream }\end{array}$




\section{INTRODUCTION}

The focus of this thesis is to find a passive way to reduce drag on a bluff body. A bluff body is any body whose dominant source of drag is pressure drag. For all of the work done in this thesis and the work done previously [1-2], the bluff body consisted of a rectangular cross section with a blunt trailing edge and either an elliptical or a circular leading edge.

Engineers are always looking for ways to reduce drag on aerodynamic systems. This includes more than just aircraft. One main reason for drag reduction on a vehicle is to improve fuel efficiency and reduce operational costs. This goal relates to this experiment because most cargo transport vehicles closely resemble a bluff body with a fairly aerodynamic leading edge and a relatively blunt trailing edge. Currently there are methods in use to reduce drag such as the tapered extensions on the back of large semitrailers. The goal of this experiment is to improve upon these methods.

A bluff body poses many interesting aerodynamic problems because of its geometry. Due to its many uses a bluff body is still a necessity for many modern designs. The circular or elliptic leading edge can be considered to have good aerodynamic properties, but the blunt trailing edge is the prominent source of pressure drag. This large source of drag is caused by flow separation and is further increased by a phenomenon called Von Kármán Vortex Shedding. Kármán Vortex Shedding is caused by the sudden separation of the flow around the body. Because of the ninety degree turn at the trailing edge, the flow instantaneously separates, forming the shear layer, and then subsequently rolls-up into vortices behind the body. The vortices from the four sharp edges are what create an 
increase in suction behind the body, increasing the drag. By attenuating the vortices the drag can be reduced.

Similar experiments deal with distributed forcing of the trailing edge of the body. Distributed forcing is a type of active flow control that uses the blowing or suction of air into the flow at the trailing edge to control vortex shedding. Distributed forcing in the trailing edge has proven to reduce drag on many types of bodies, but thus far has not proven completely successful on a bluff body.

This thesis will look into ways of reducing the drag caused by a bluff body through wind tunnel testing and the addition of passive tabs on the model's trailing edge. These tests include surface pressure measurements, total drag measurements via a sting balance, boundary layer measurements, and hotwire velocity fluctuation measurements. 


\section{LITERATURE REVIEW}

The beginning of this series of experiments started at Cambridge University by an engineer named Bearman [1-2]. Bearman did two experiments to reduce bluff body drag, the first used splitter plates [1] and the second used base bleed [2]. In Bearman's first experiment, a splitter plate was connected to the trailing edge of the model and ran parallel to the flow. The plate spanned the entire width of the model and essentially separated the wake from the top and bottom surfaces of the model. Bearman's results showed that the addition of the splitter plate was sufficient to increase the base pressure coefficient of the model. This confirms the theory that removing, or prolonging, the vortex shedding was a feasible way to reduce the overall drag. In Bearman's second experiment [2] he was able to reduce the vortex shedding at high speeds over a bluff body by having compressed air escape from the base of the model.

These experiments were then continued by a group of Korean engineers led by Hyungmin Park et al. [3] who did wind tunnel testing on a two dimensional bluff body. This model was two dimensional because it spanned the entire width of the wind tunnel. Their results showed that the drag could be reduced by using passive trailing edge tabs at all three of the test speeds. They also did large eddy simulations on the computer to validate their theory for the mechanism of drag reduction.

Using the large eddy simulations, Park et al. [3] were able to show that the increase in the wake width was the main method for reducing vortex strength. Without tabs the wake was the same width along the entire model. By adding the tabs it created an area of thicker wake width behind the tabs. The change in wake width across the span of the trailing edge created a mixing factor within the wake. This mixing prolonged the 
formation of the vortex, increased the formation length, and reduced its effect on the body. The increase in wake width decreased the interaction between the top and bottom vortices and caused an increase in base pressure, thus reducing drag. These results are shown visually in Park's paper.

At the same time, the first bluff body experiment was being conducted at Cal Poly by James Knight and Jin Tso [4-5]. This experiment focused on drag reduction on a bluff body by use of passive surface roughness at the leading edge. Knight's research also measured the trailing edge boundary layer response due to the various roughness of trip tape at the leading edge. In his research Knight was able to show that with increasing surface roughness, the base pressure was increased which decreased the base drag. This however did not hold true at higher Reynolds numbers.

To expand upon Park's two dimensional results, a former Cal Poly student, Jarred Pinn [6], created a three dimensional model to test. Pinn had a cylindrical leading edge with a 4 to 1 cross sectional aspect ratio. His tests proved that even in three dimensions the passive tabs led to a significant reduction in drag but only for the two lower speed cases. The high speed test showed that the tabs actually increased the overall drag of the body. Because of this increase in drag, it is believed that the original equations used by Park to determine the tab height are invalid at high speeds for a 3D model. Instead it is now believed that the tab height is more dependent on the thickness of the boundary layer instead of just the model width. Two other Cal Poly students, Paul Innes and Charles Carlson [7], did a senior project to validate Pinn's base pressure results. While they were not able to reproduce the same curves they were able to come to the same conclusion: that the tabs do increase the average base pressure. 
Another former student, Ethan Erlhoff [8], tested the distributed forcing in the trailing edge of a bluff body. For his model Ethan used a high aspect ratio 3D body with an elliptical leading edge with a blunt trailing edge. This model had four slots in the top and bottom of the trailing edge that were then connected by internal ducts to an electric fan that would control the blowing or suction through the slots.

Erlhoff's results did show significant reductions in drag, but still needed energy input to operate the fans. For the lower test speeds this was not a problem because the energy saved by the drag reduction was greater than the electrical energy that was added. Unfortunately, to get drag savings at higher speeds the electrical energy input to the system far outweighed the drag savings. These results showed that active flow control for a bluff body was plausible but with the current technology it does not outweigh the energy costs.

Because of the success of Pinn's study on the effectiveness of end plate tabs in attenuating vortices and reducing drag at lower Reynolds numbers, this thesis looked into ways of reducing the drag caused by a bluff body with square base with the addition of passive tabs on the model's trailing edge. 


\section{EXPERIMENTAL APPARATUS}

This section describes the design and implementation of the experimental model as well as the testing equipment. It is also discussed how the testing equipment was calibrated and configured. Calibration results are discussed in the results and discussion section.

\subsection{Experimental Model}

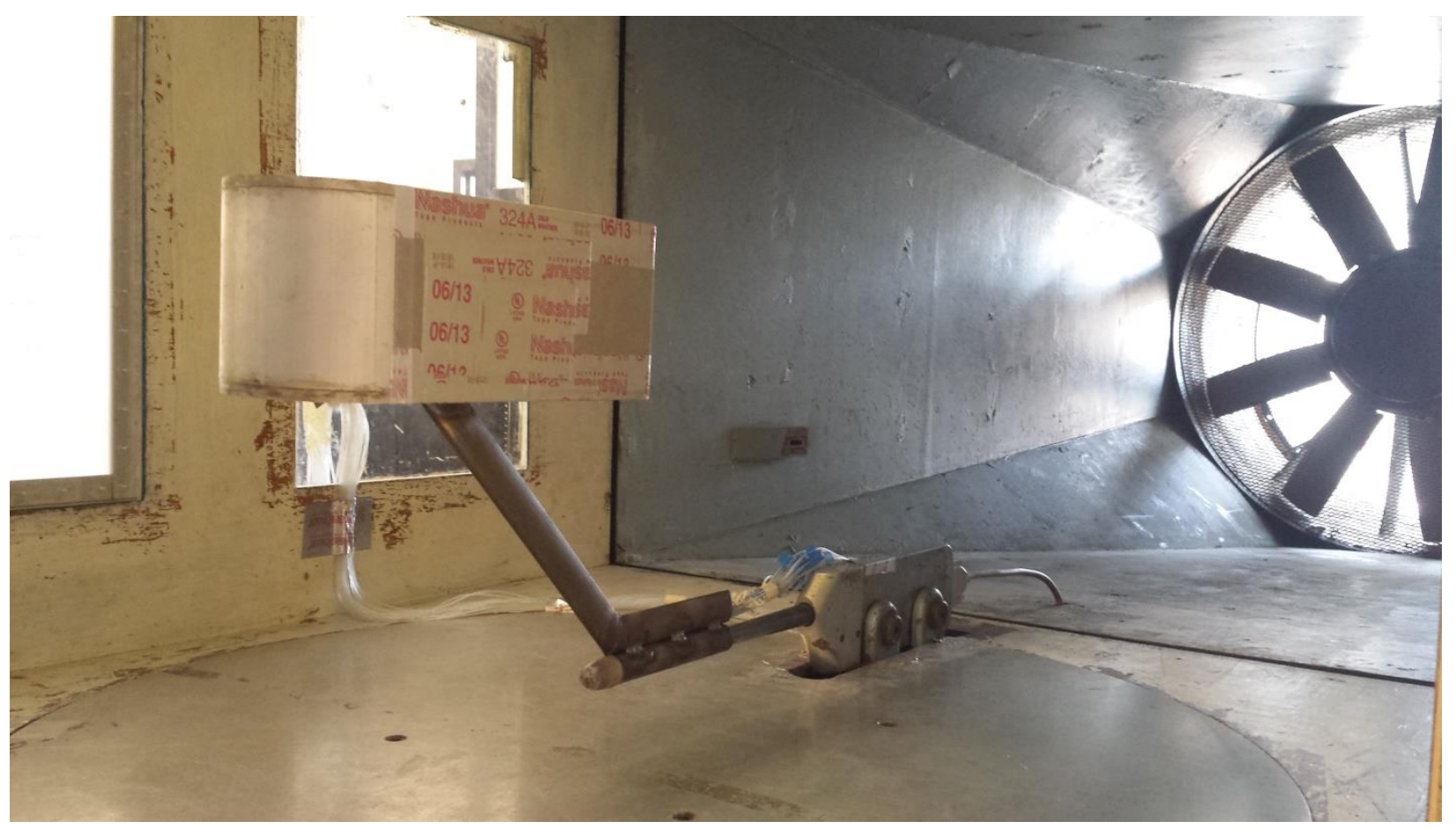

Fig. 1. Experimental model mounted in wind tunnel.

The model that was used for this experiment is very similar to previous experiments $[3,4]$ and can be seen mounted in the wind tunnel above in Fig. 1 . Each of the previous models was slightly different and this model is the next step in the progression to prove the usefulness of the passive tabs. The Park et al. model [3] was two dimensional, both Pinn's model [6] and Knight's model [4-5] were three dimensional, but still had high aspect ratios. The current model is similar in construction to Pinn's in that they were both 
made out of 6061 aluminum with a circular nose, but this model has a cross sectional aspect ratio of 1 to 1 . A schematic of the model can be seen below in Fig. 2. As shown it has a cross section with a height and width of $0.127 \mathrm{~m}$ (5 inches). The leading edge of the model is composed of a half cylinder with a radius of $0.0635 \mathrm{~m}$ ( 2.5 inches). The length of the entire model was chosen to be similar to that of Pinn's experiment and overall is $0.2984 \mathrm{~m}$ (11.75 inches) long.

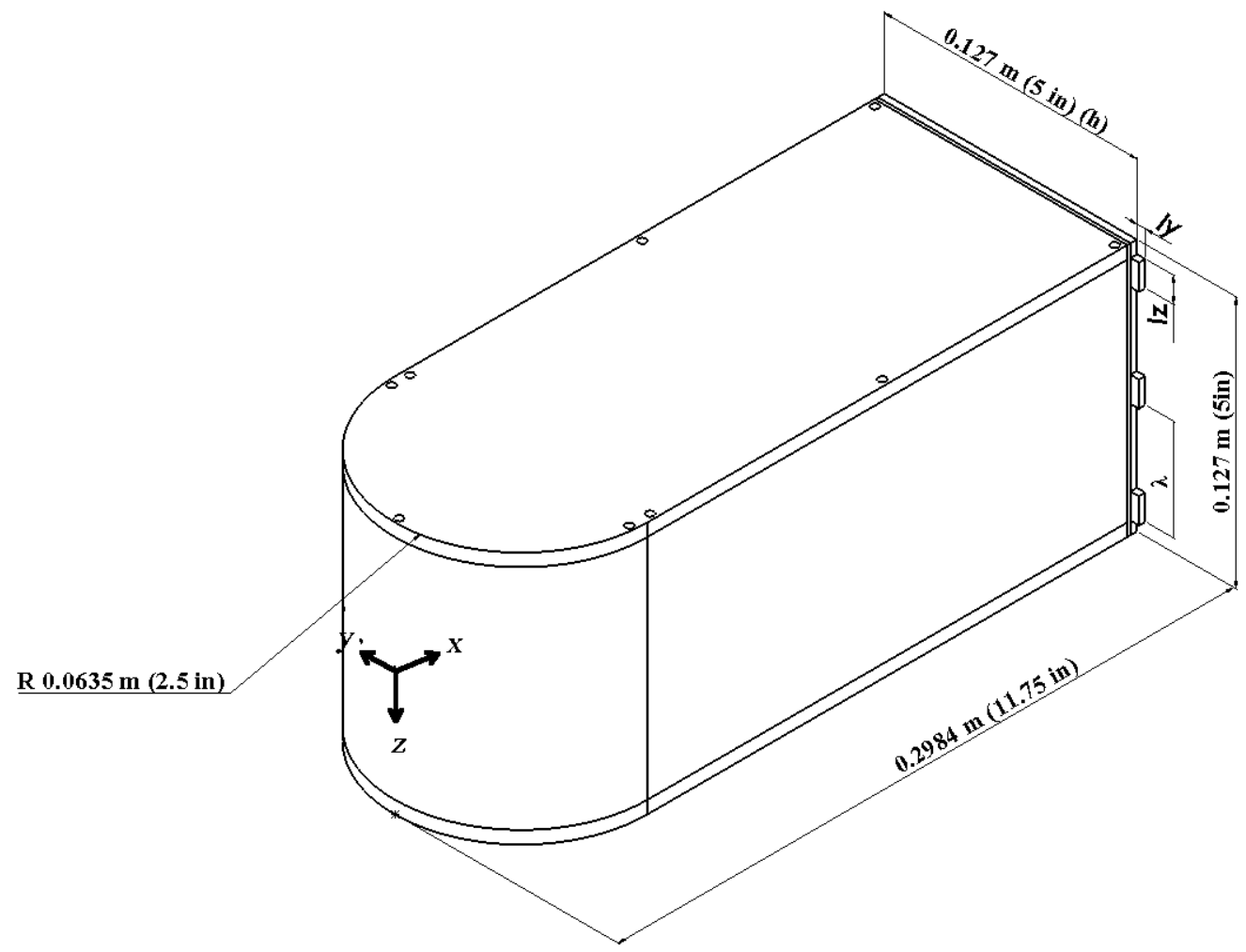

Fig. 2. Schematic of test model.

Along with a different aspect ratio, this model improves upon previous tab designs. The previous two experiments $[3,6]$ had "static" tabs that were dependent only on the width of the model. Because of Pinn's results at high speeds [6], this model was designed with "adaptive" tabs. This means that instead of the tab height being dependent on the width of the model; it will now change depending on the momentum thickness. As 
discussed later, the tabs are designed to protrude into the flow less as the speed increases. This adaptive tab height can be seen in the schematic in Fig. 4 below. Based on tests discussed later in this paper, the tabs were set to protrude $0.0059 \mathrm{~m}, 0.0043 \mathrm{~m}$, and $0.0038 \mathrm{~m}(0.2338,0.1687$, and 0.1487 inches $)$ into the flow for speeds of 10,20 , and 30 $\mathrm{m} / \mathrm{s}(32.8,65.6$, and $98.4 \mathrm{ft} / \mathrm{s})$ respectively. These will be referenced later as high, medium, and low tabs for the 10,20 , and $30 \mathrm{~m} / \mathrm{s}$ speeds respectively.

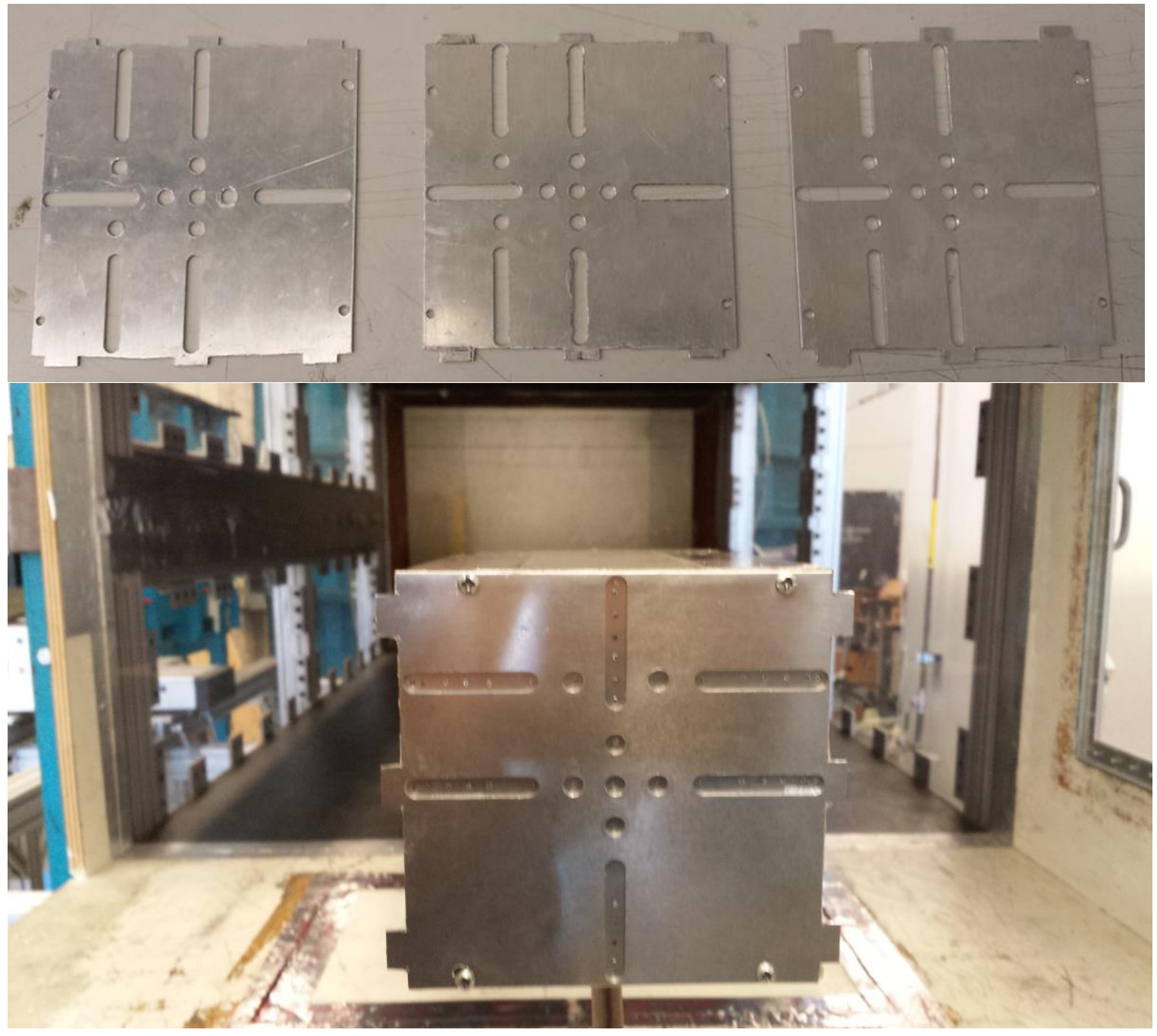

Fig. 3. Removable rear plates and rear plate on model in wind tunnel.

As shown in Fig. 3 the tabs are built into three removable rear plates that screw into the trailing edge of the model. These removable plates allow for the tab heights to be 
easily reconfigured. Using removable tabbed plates, the rear plate of the model does not need to be opened and the tubing does not need to be reconfigured as in previous experiments. This improvement allows for quicker and easier setup changes as well as more consistent data while reducing the probability of tubing leaks. In the tab schematic in Fig. 4 below, it shows three different numbers on the tabs. These are the tab heights for each test, not for each tab. For all three test configurations, all six tabs were the same height. The tab spacing was determined by keeping the tabs as similar as possible to previous experiments. In both Park et al. and Pinn's experiment the tabs were sized based on a ratio of base area to tab area. Because this model has a similar area to Pinn's the width of each tab was set equal to Pinn's tab width. The tab spacing was then determined based on having an already set tab width and then equally spacing the three tabs across the body. 


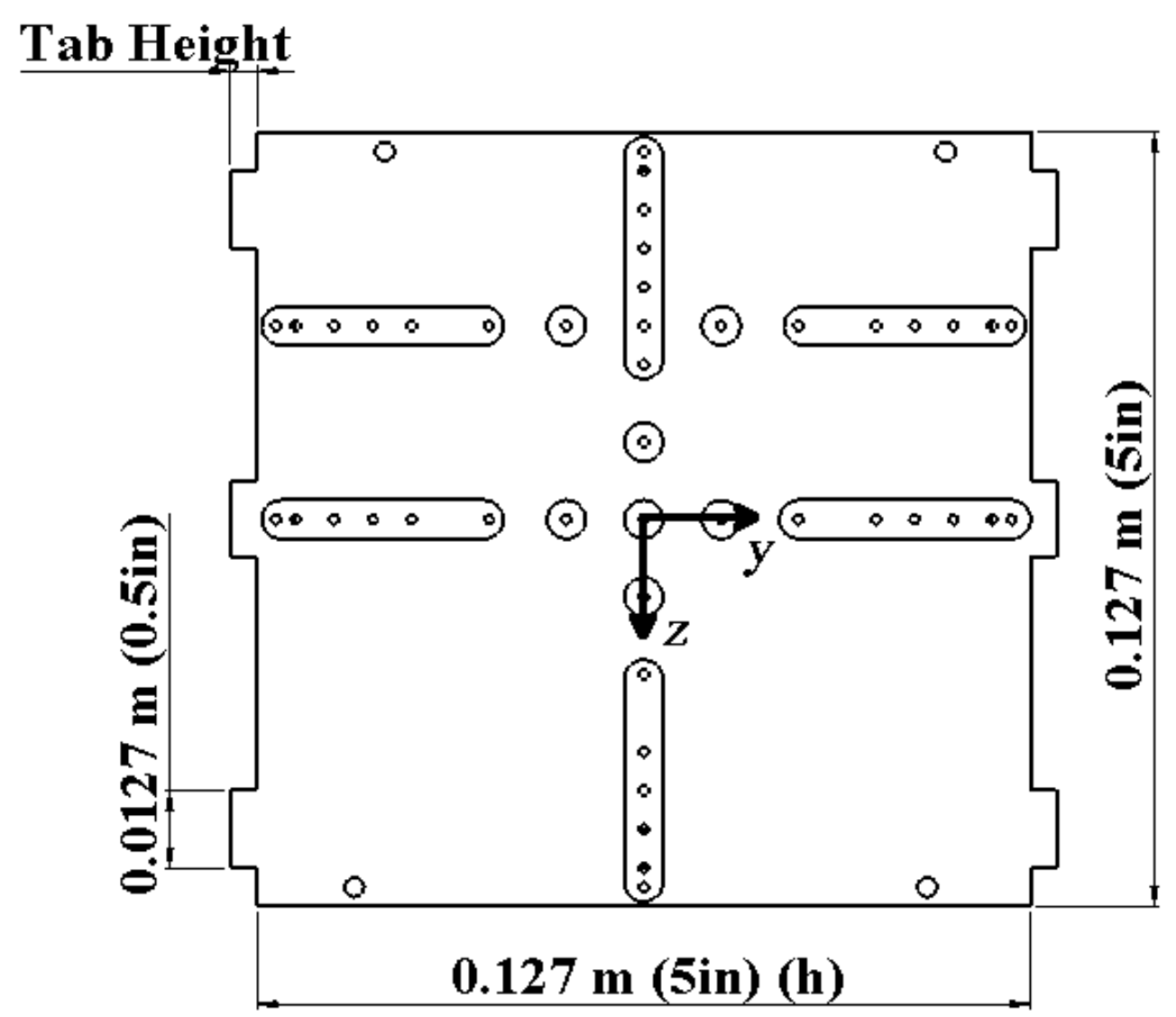

Fig. 4. Rear plate schematic. Tab heights are $0.0059 \mathrm{~m}, 0.0043 \mathrm{~m}$, and $0.0038 \mathrm{~m}(0.2338$, 0.1687 , and 0.1487 inches).

Fitted inside the model are sixty $0.0016 \mathrm{~m}\left(1 / 16^{\text {th }}\right.$ inch $)$ stainless steel pressure ports

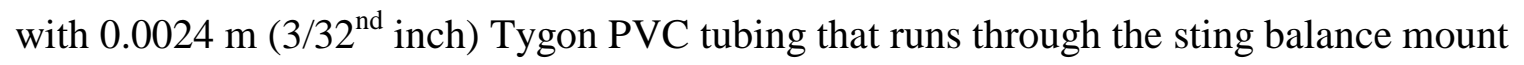
and into a pressure transducer. This data is then sent to the computer for analysis. The pressure port distribution can be seen in Fig. 5. Previous models had the pressure ports run all the way around the nose but only one side is necessary to show the profile around the body. The inner diameter of the Tygon tubing is smaller than the outer diameter of the pressure ports and aluminum tubing to create an interference fit. This was done for ease 
of setup and to reduce the risk of leaks, whereas in previous experiments lock wire was required to connect the tubing to the ports [6].
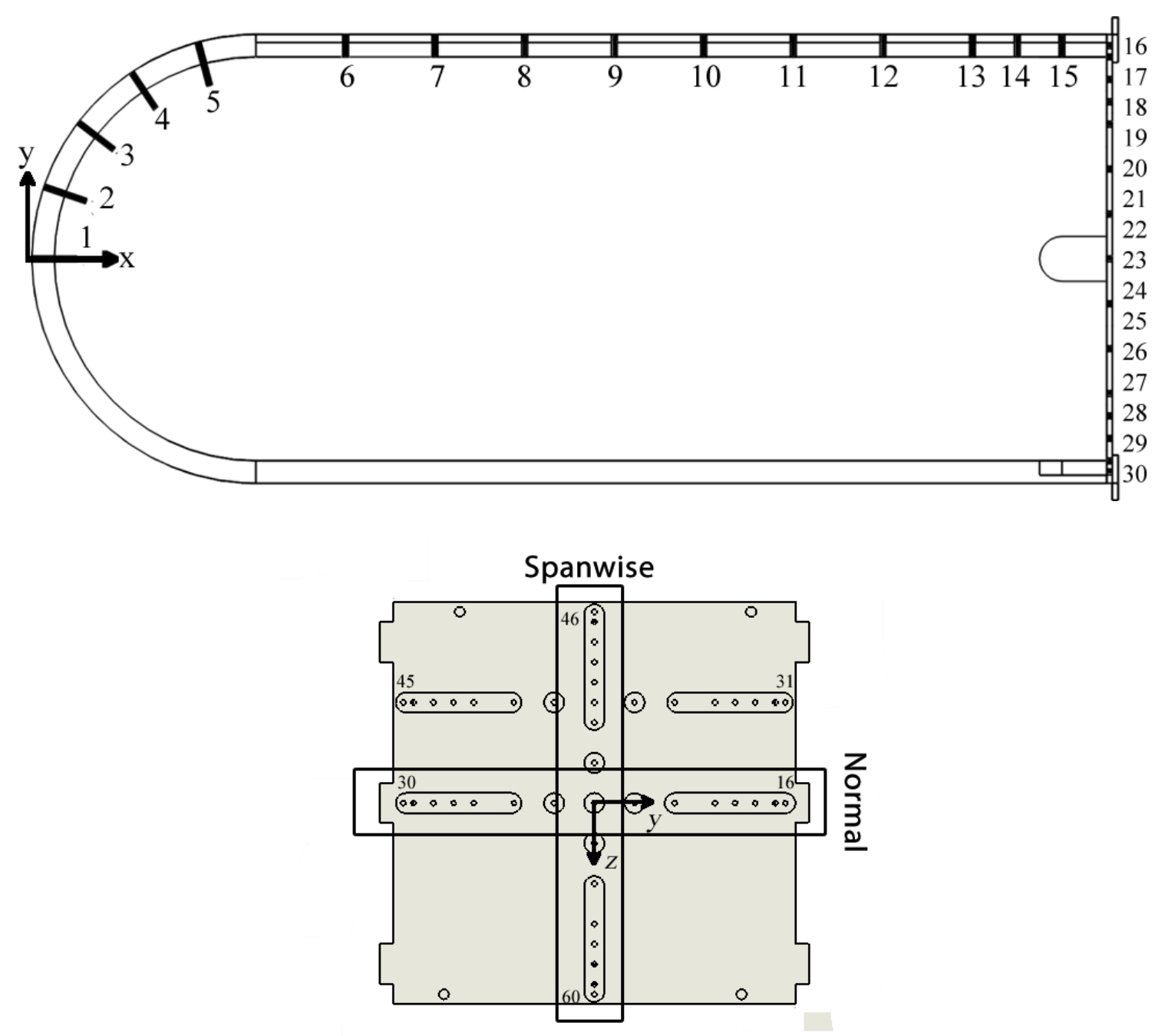

Fig. 5. Side and back plate pressure port distribution schematic.

The ports were spaced in a way that maximizes the amount of relevant data. This is done by putting more pressure ports near the edges of the model and fewer in the middle. Towards the edges there is a $0.0032 \mathrm{~m}\left(1 / 8^{\text {th }}\right.$ inch $)$ spacing between each port and towards the center there is a $0.0127 \mathrm{~m}(1 / 2 \mathrm{inch})$ spacing between ports. This allows for a more accurate view of the influence of the vortex shedding on the pressure distribution. 
To ensure that the influence of the vortex shedding on the pressure was captured in its entirety, there are three rows of pressure ports on the base of the model. There are two rows that run perpendicularly to the tabs along the normal direction. This is to allow the measurement of the vortex over the tabs and between the tabs. The third row that runs parallel to the tabs in the spanwise direction is to measure the vortex shedding from the sides without tabs.

The model is mounted to the wind tunnel via a custom strut shown in Fig. 6. This strut was made out of a hollow 4130 steel tube that was formed as a symmetric airfoil to maintain smooth flow. At the top of the strut, as shown below, there is a plate with four screws that screw directly into the test model. The bottom has two hollow cylinders welded together. The bottom cylinder is where the strut mounts to the sting balance in the wind tunnel. The top cylinder is to guide the internal tubing. As shown in Fig. 6 there are thirty aluminum tubes that run through the airfoil section of the strut. These tubes are used to connect the pressure ports inside the model to the scanivalve outside of the wind tunnel. The width of the airfoil section limited the number of pressure tubes to thirty. This space constraint is what limited the amount of pressure ports per row to fifteen. With fifteen pressure ports per row, two rows can be tested simultaneously. 


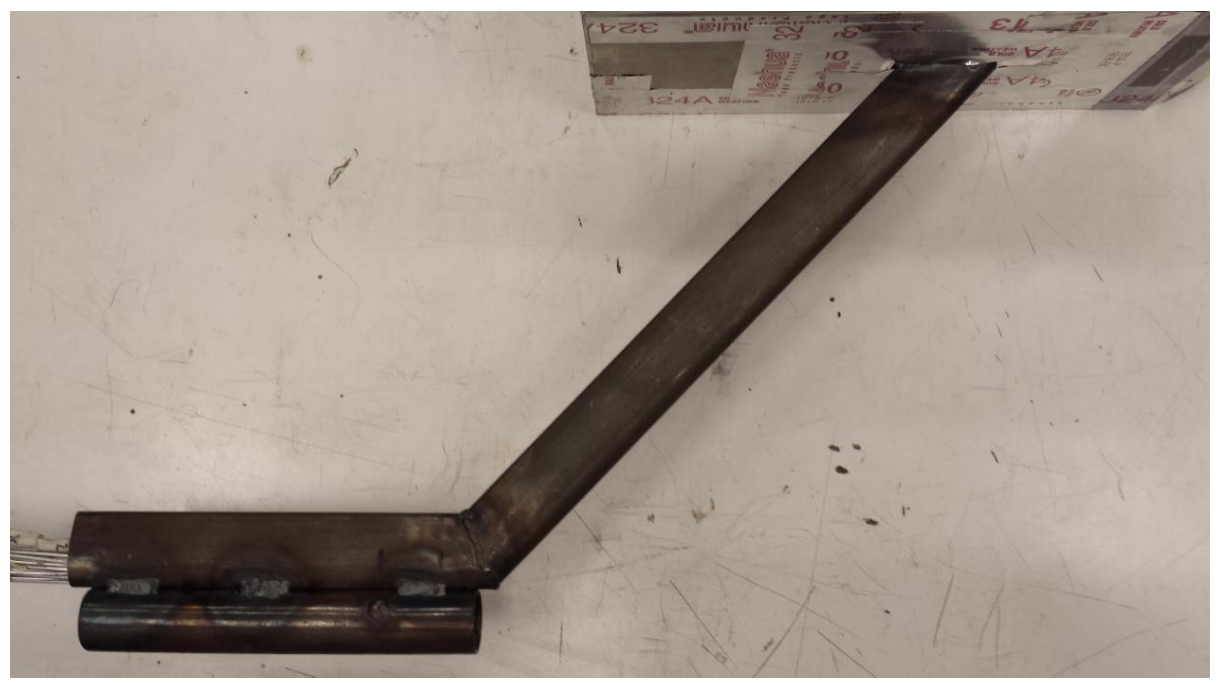

Fig. 6. Hollow strut mount attached to experimental model.

\subsection{Wind Tunnel}

All of the tests for this experiment were done in Cal Poly's subsonic wind tunnel. This tunnel is an open circuit wind tunnel that consists of a 3.66 meter by 2.74 meter (12 feet by 9 feet) inlet with a 1.22 meter by 0.91 meter ( 4 feet by 3 feet) test section. Built into the floor of the test section is a sting balance built by Aerolab that is used to record the forces on the model. The flow is kept laminar by placing the fan behind the test section and pulling the air through the test section. This fan is a nine blade fixed pitch fan that is driven by a 150 horsepower electric motor that is controlled by a SquareD Altivar66 manual variable frequency generator. The wind tunnel is in an enclosed room, but the door behind the wind tunnel opens so the air can be expelled out of the room. There is another vent in the ceiling by the inlet which brings in air from the outside. The tunnel was constructed in 1974 by Professor Jon Hoffman with help from other faculty and students. The tunnel was built primarily out of wood and has removable test sections to allow for many different types of experiments. One of these removable sections can be seen on the left of Fig. 7. 


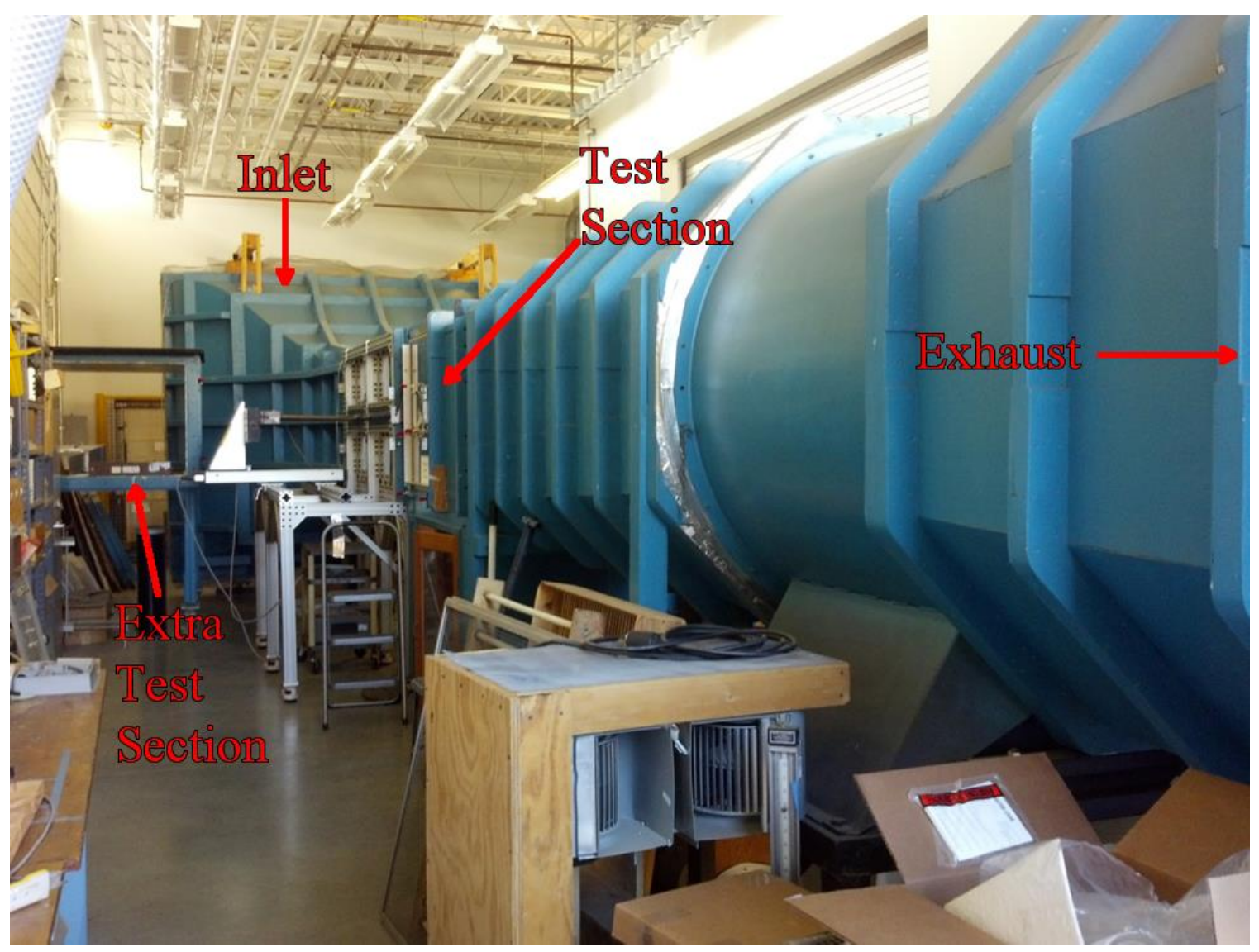

Fig. 7. Cal Poly wind tunnel laboratory. On the left there is a removable test section. Next to the removed section is the inlet followed by the attached test section and then the exhaust nozzle.

During testing the VFD (Variable Frequency Drive) for the tunnel broke down and the tests had to be completed with two different motors. The first motor was an Elliott Co. $150 \mathrm{hp}$ three phase electric motor and was used to complete the boundary layer testing as well as the tunnel calibration and longitudinal pressure profile tests. The tunnel calibration test was required because the velocity measurements used to control the tunnel were for the inlet not the test section.

The second motor was an $125 \mathrm{hp}$ three phase electric motor controlled by a AllenBradley VFD and was used to complete the base pressure, total drag, and hotwire tests. 
Instead of doing a tunnel calibration for these tests, a Pitot-static probe was inserted into the tunnel in front of the model to measure the flow velocity in the test section. The probe was placed in a mid-span location about $5 \mathrm{~h}$ ahead of the model and $3 \mathrm{~h}$ below the model, outside the boundary layer on the wind tunnel wall. No other major changes were made to the tunnel during the course of this experiment other than changing motors and controllers.

\subsection{Scanivalve}

To measure the pressure along the top plate and base of the model, tubes were run from the pressure ports along the model to the scanivalve outside the wind tunnel. The scanivalve itself was a ZOC33/64P-X1 pressure scanner. It has the ability to read 64 pressure ports simultaneously with the added input of $448 \mathrm{kPa}(65 \mathrm{psi})$ shop air as a reference pressure. The first 32 ports have an input range of \pm 10 inches of water $(2.491$ $\mathrm{kPad}, 0.3613 \mathrm{psid}$ ) with an accuracy of $\pm 0.15 \%$ full scale reading while the remaining 32 ports have an input of $\pm 6.895 \mathrm{kPad}$ ( 1 psid) and an accuracy of $\pm 0.12 \%$ full scale reading. The scanivalve can be seen in the center of Fig. 8 along with the adapter plate and vacuum gun. 


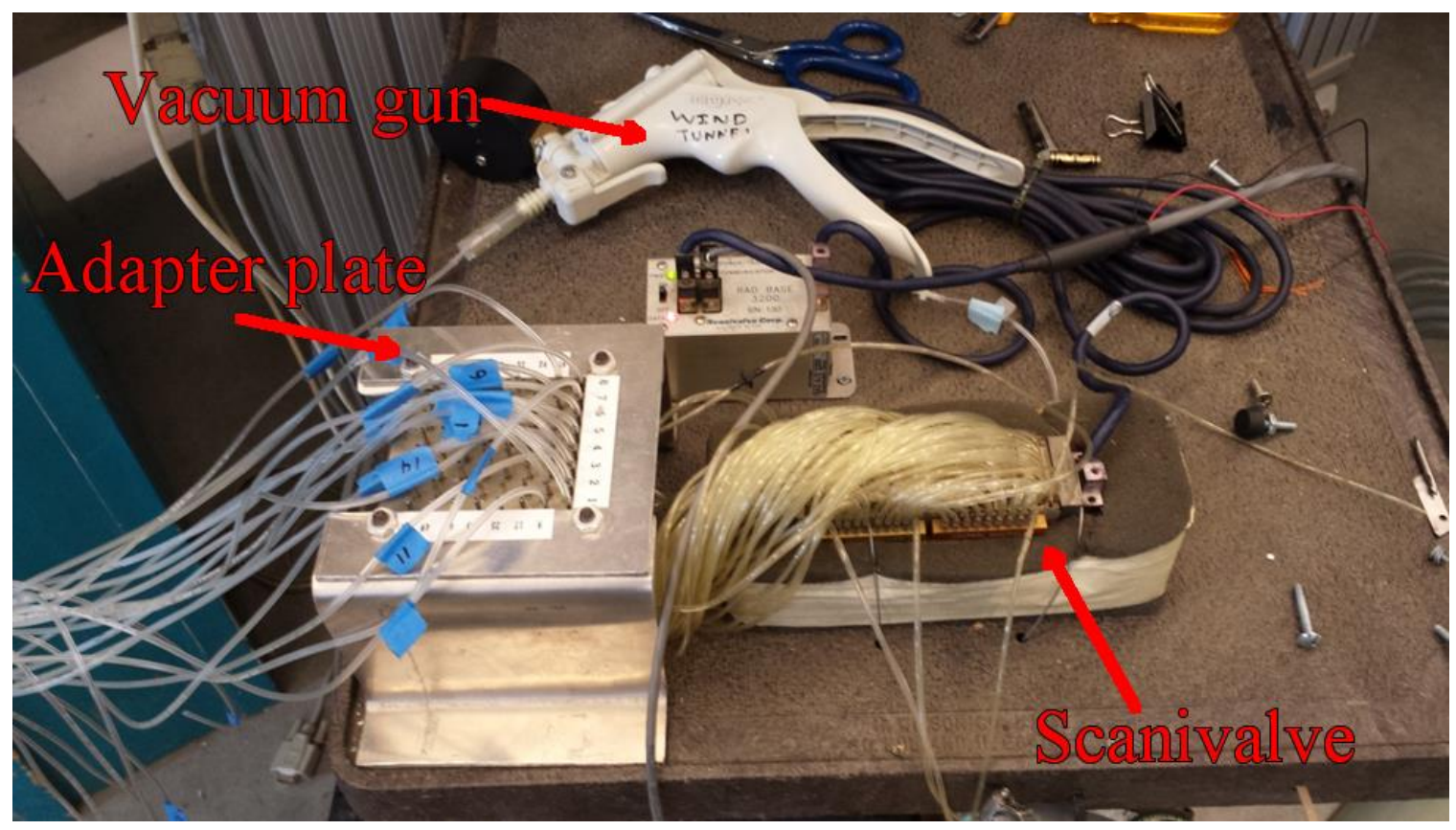

Fig. 8. Adapter plate (left), ZOC33 Scanivalve (right), and vacuum gun (top).

To make testing easier, an adapter plate was implemented between the model's tubing and the scanivalve to adapt the size of the tubing. This allows for the ports connected the scanivalve to remain untouched and allow for more standardized tubing sizes to be used on the experimental models.

Tubing was run from the ports along the model, through the hollow airfoil strut, out of the wind tunnel and into the adapter plate. From there, smaller tubing was run into the ZOC33 where the pressures were converted into voltages. These voltage values were then sent to and amplified in a RAD3200 analog to digital converter. This data was then sent to a computer running the RadLink V2.10 software that read and stored the data from the pressure tests. The raw data was then processed using MATLAB. The internal tubing can be seen in Fig. 9. 


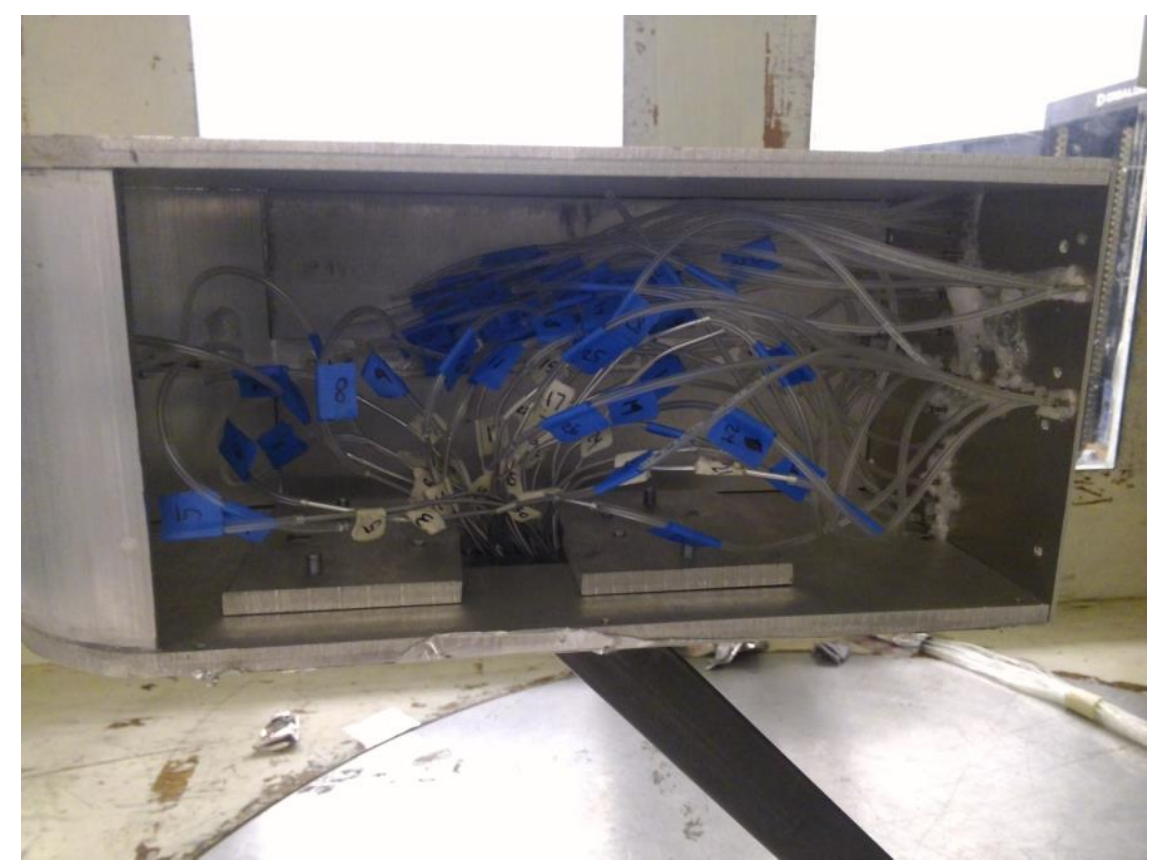

Fig. 9. Model internal tubing on sting balance.

The scanivalve was calibrated using a U-shaped manometer filled with water shown in Fig. 10. A constant pressure was applied to the manometer and the computer tested each port to make sure it was reading accurately.

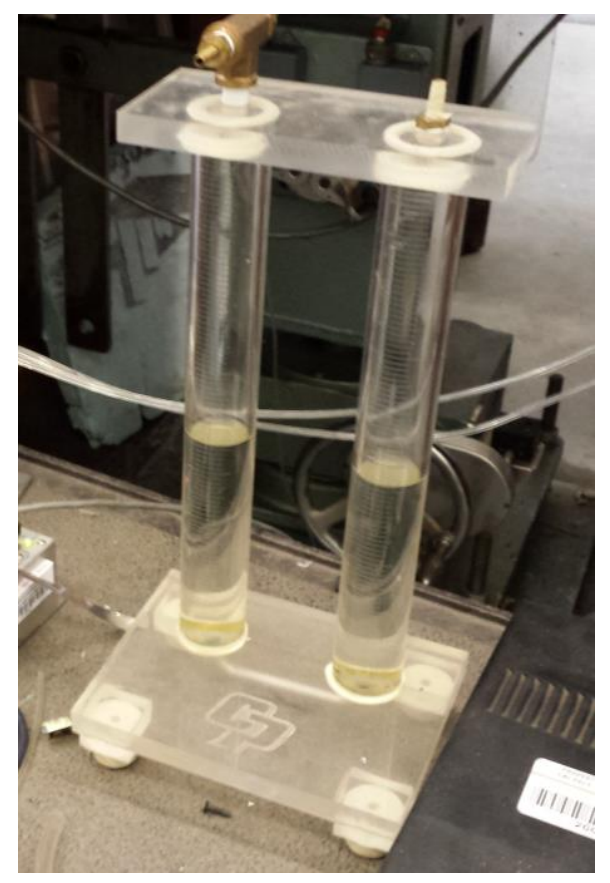

Fig. 10. Manometer used to calibrate the scanivalve. 
Between each test the model was checked for leaks. Though it was unlikely the tubes came loose between tests, as they are not exposed to the flow, leak checking was done to ensure accurate data. Leaks were checked by unplugging the tubing from the adapter plate and connecting it to a vacuum gun. The pressure port on the model was then covered by a finger and a pressure was applied via the vacuum gun. If the pressure stayed constant then there were no leaks. If the pressure returned to zero then that tube was checked immediately for the source of the leak.

\subsection{Sting Balance}

The model was held in place using a sting balance that was built into the floor of the wind tunnel. This sting balance has the ability to not only move vertically in the tunnel but also change the angle of attack and yaw angle of the test model. This is done manually via the controller outside the wind tunnel or from the computer using LabView. The controller can be seen in Fig. 12 below. For these tests the angle of attack and yaw angle were always set to zero relative to the flow. Also built into the sting balance are sensitive strain gauges that allow for the measurement of all six degrees of freedom. For this experiment the axial force was measured to calculate the total coefficient of drag.

The sting balance sent a voltage from each of the six internal strain sensors through an amplifier inside the controller and was then read by a data acquisition card. The data was then sent to the computer and recorded using a custom LabView program. The sting balance also acts as the mount that holds the test model in place. This was achieved via a long tapered rod with a threaded hole at the end. The model mount slid over the tapered rod and was pulled tight using a screw in a threaded hole causing a friction fit on the tapered rod. 
To calibrate the sting balance, small weights were used that ranged from 0.4536 to $2.268 \mathrm{~kg}$ ( 1 to 5 pounds) in increments of $0.4536 \mathrm{~kg}$ (one pound). The weights were hung off of the tip of the sting balance and over a pulley to calibrate the axial force on the sting. The voltage for each weight was recorded. The calibration setup can be seen in Fig. 11. The calibration produced a linear correlation between the weights and a trend line was calculated. The equation of the trend line was later used to find the forces on the test model.

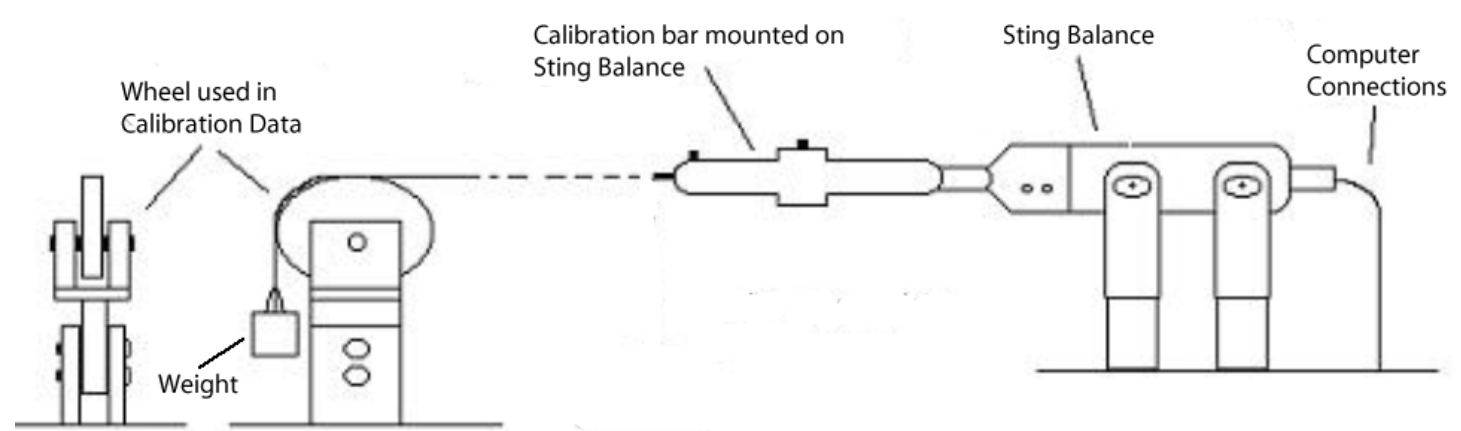

Fig. 11. Sting balance calibration schematic.

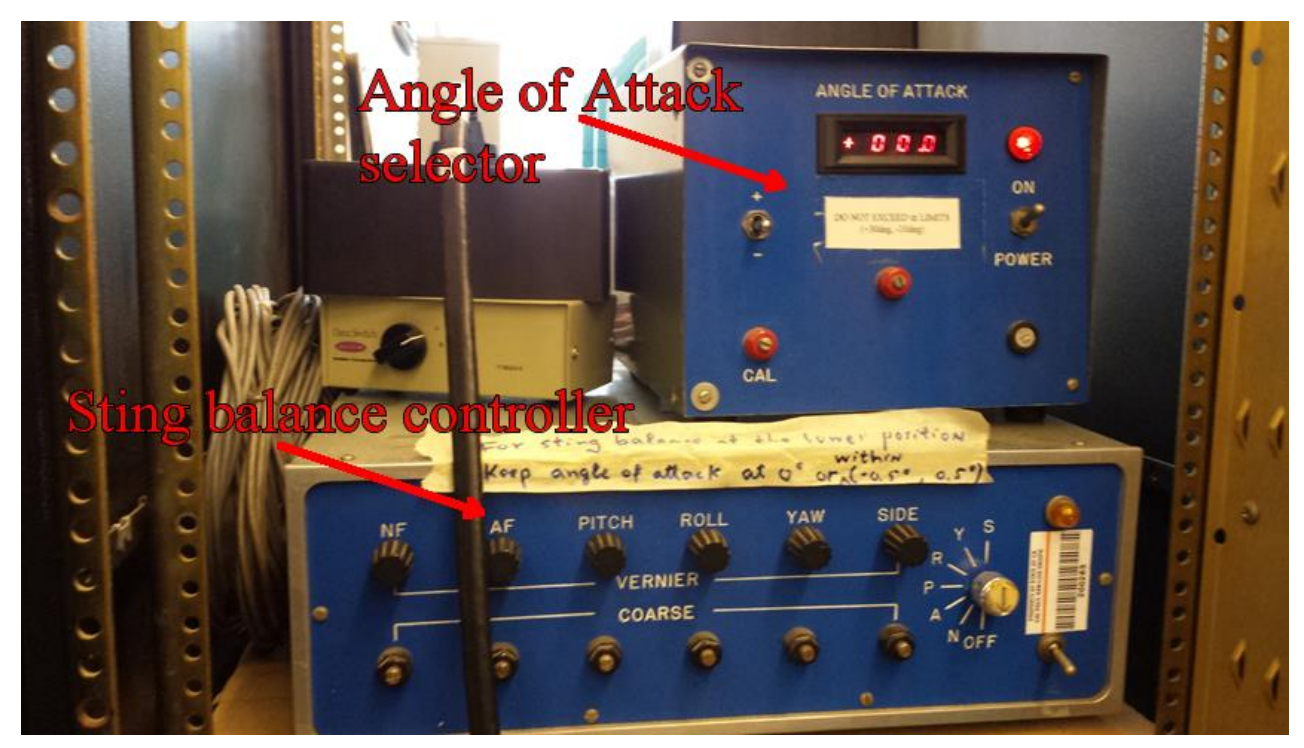

Fig. 12. Sting balance controller. 


\subsection{Traverse System}

Due to the need for high precision measurements inside the wind tunnel, the Velmex Traverse system shown in Fig. 13 was used to move the hotwire and boundary layer probes laterally through the tunnel. The Velmex traverse uses an electric stepper motor and screw to slowly move a cart linearly. A metal plate was then attached to the cart on the metal screw that extended into the wind tunnel. The traverse was then mounted to a custom removable window that clamps into a slot on the side of the wind tunnel. The traverse window can be seen mounted to the tunnel in Fig. 14. The window only sealed on the top and bottom edges so aluminum tape was used to seal the remaining two sides from the inside. A second aluminum arm was screwed to the metal plate on the traverse to move the hotwire probe and boundary layer probe forward in the tunnel. The screw on the traverse system was set up with a correlation of 4000 steps from the motor to rotate the screw ten times and move the cart forward $0.0254 \mathrm{~m}$ (one inch). This was measured and calibrated using a caliper prior to testing.

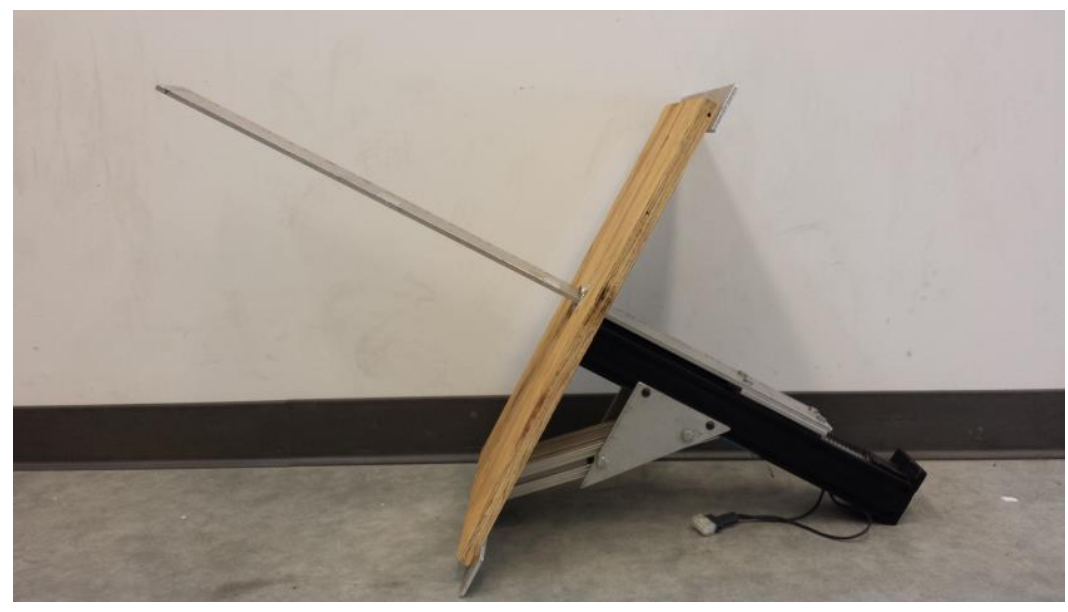

Fig. 13. Traverse window. 


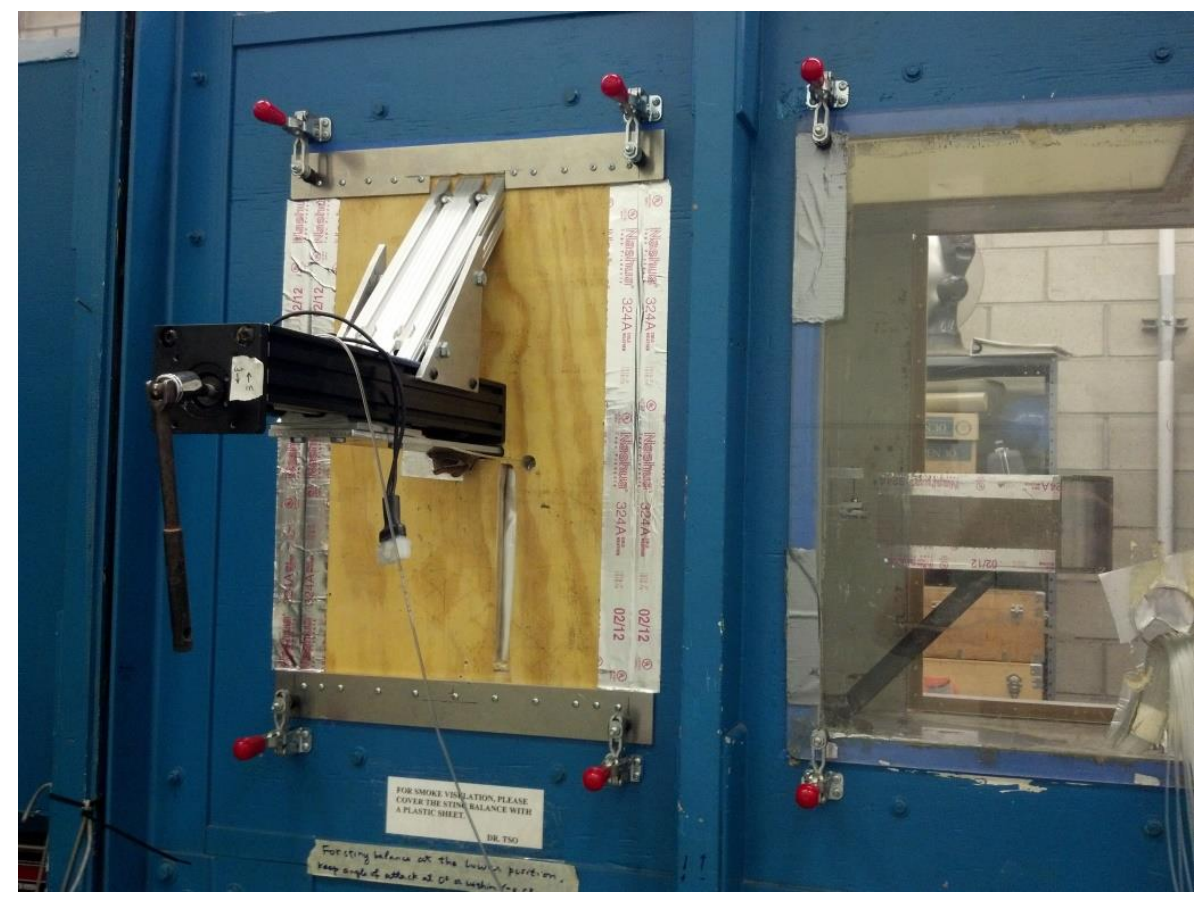

Fig. 14. Traverse mounted to side of wind tunnel.

\subsection{Boundary Layer Probe}

The boundary layer probe is a very small total pressure probe that was used in conjunction with the traverse described above and a static port (port 15 in Fig. 5) on the rear edge of the test model. The probe was a United Sensor Model BA-025-12-C-11-650 probe which has a sensing head diameter of $0.0006 \mathrm{~m}$ (0.025 inches), with a flattened measuring orifice of $0.00028 \mathrm{~m}$ ( 0.011 inches) to minimize errors in total pressure measurements. This probe can be seen next to the model in Fig. 15. The boundary layer probe was used to measure the total pressure at incremental points away from the model. The pressure was measured at small increments until the probe was far enough away from the model to match the free stream total pressure. 


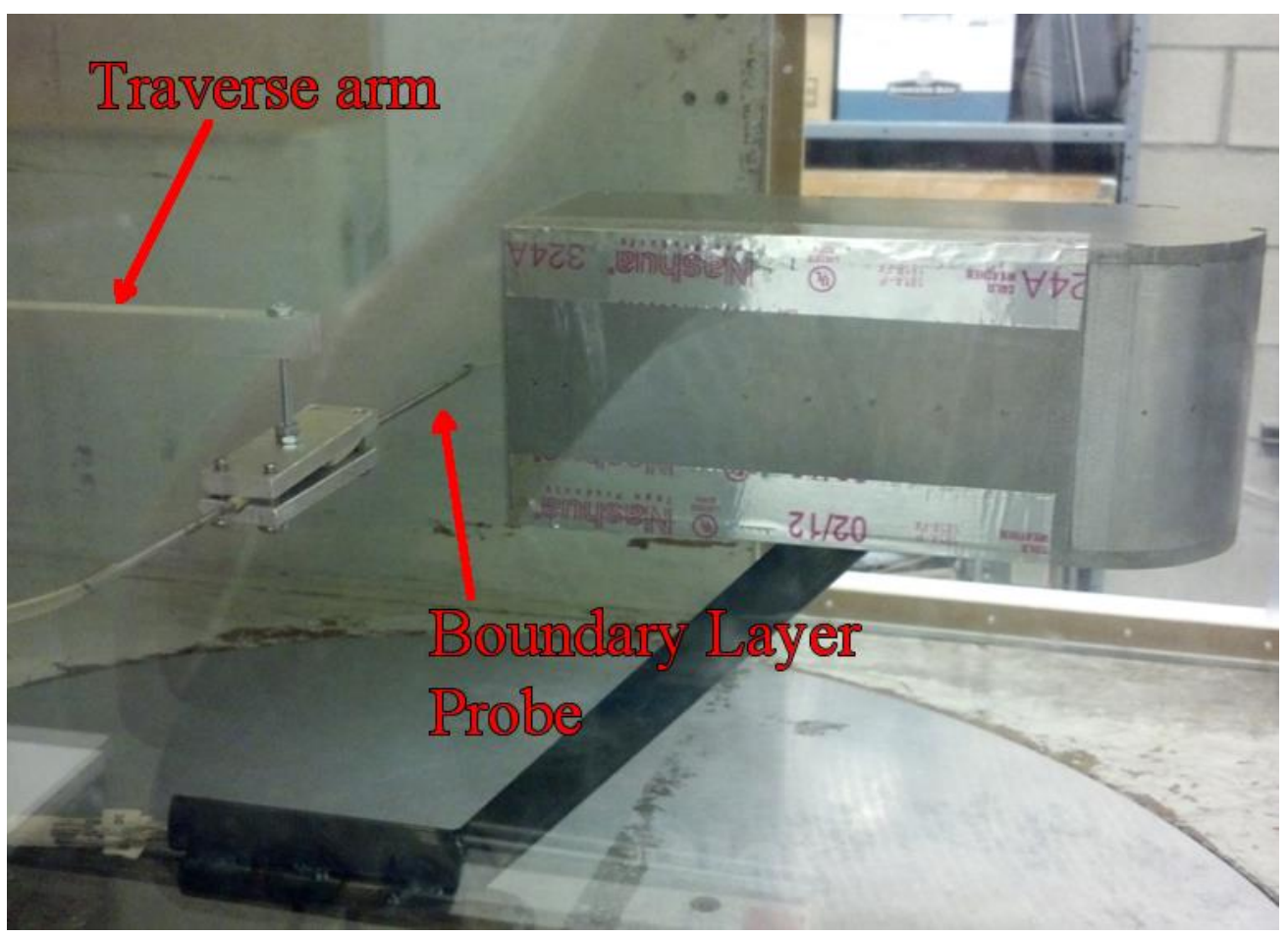

Fig. 15. Boundary layer probe at the trailing edge of the model.

\subsection{Hotwire Probe}

A hotwire probe and anemometry system was used to measure the fluctuations in the near wake behind the model. This system measured voltages from the probe and ThermalPro was used to analyze the data and produce an energy spectrum. This energy spectrum was then compared to the Strouhal number to visually show a large spike in the energy spectrum to signify the shedding of a vortex. The primary controller for the hotwire was the TSI IFA-300 constant temperature anemometer shown in Fig. 16. It supports a frequency response of $250 \mathrm{kHz}$ or greater and allows for one or two channel wire systems. For this experiment only a one wire probe was used.

To position the hotwire probe in the correct place behind the model, a second traverse system was used. This setup is similar to the boundary layer probe setup in Fig. 15. The initial setup for the hotwire used a different window and a different arm than in the 
boundary layer tests shown in Fig. 17. This window allowed for vertical movement of the arm as well as lateral movement within the tunnel. These two degrees of freedom allowed the probe to be positioned anywhere in the tunnel's cross section $0.1778 \mathrm{~m}$ ( 7 inches) behind the model. As the testing progressed it was determined that the probe could not move close enough to the model to show the vortex shedding. To fix this the traverse from the boundary layer testing was used in conjunction with the new arm to move the probe up to $0.0254 \mathrm{~m}$ (1 inch) behind the model.

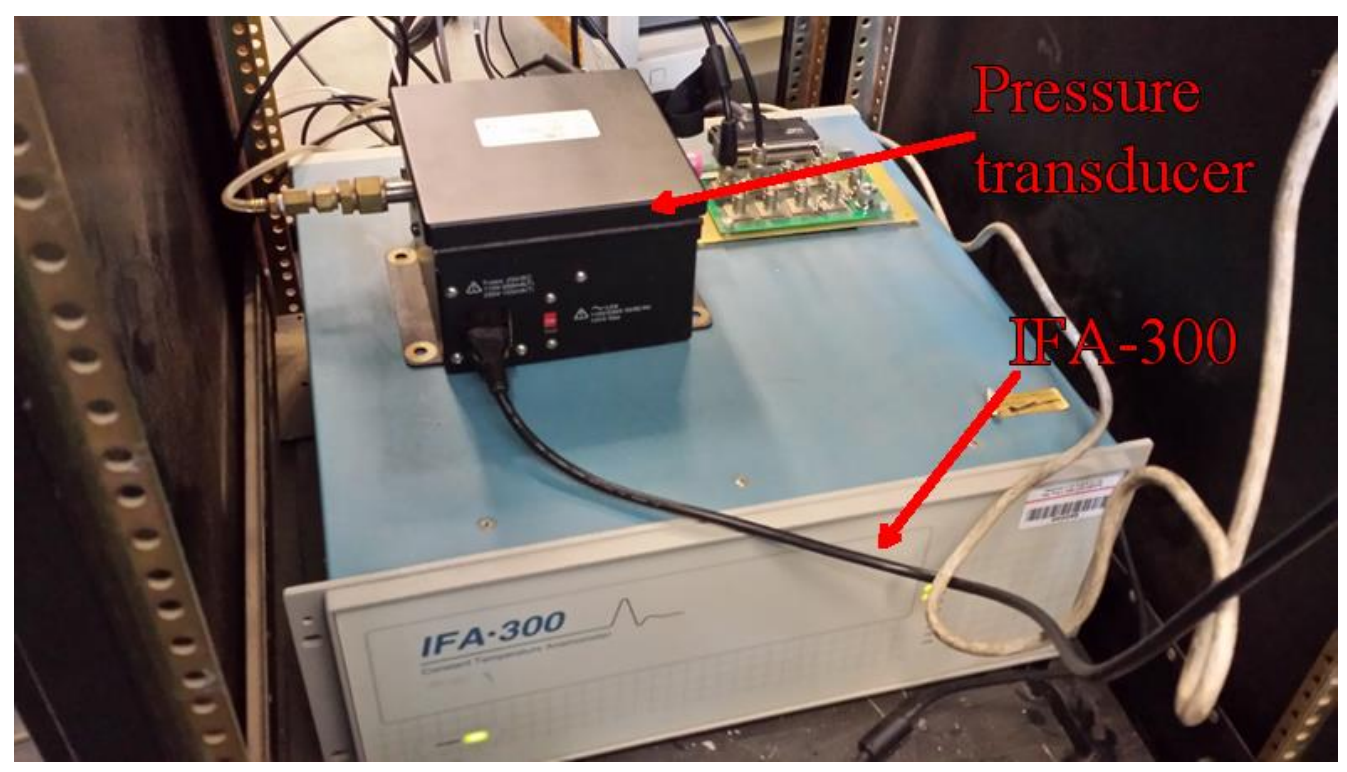

Fig. 16. IFA-300 hotwire anemometry cabinet. 


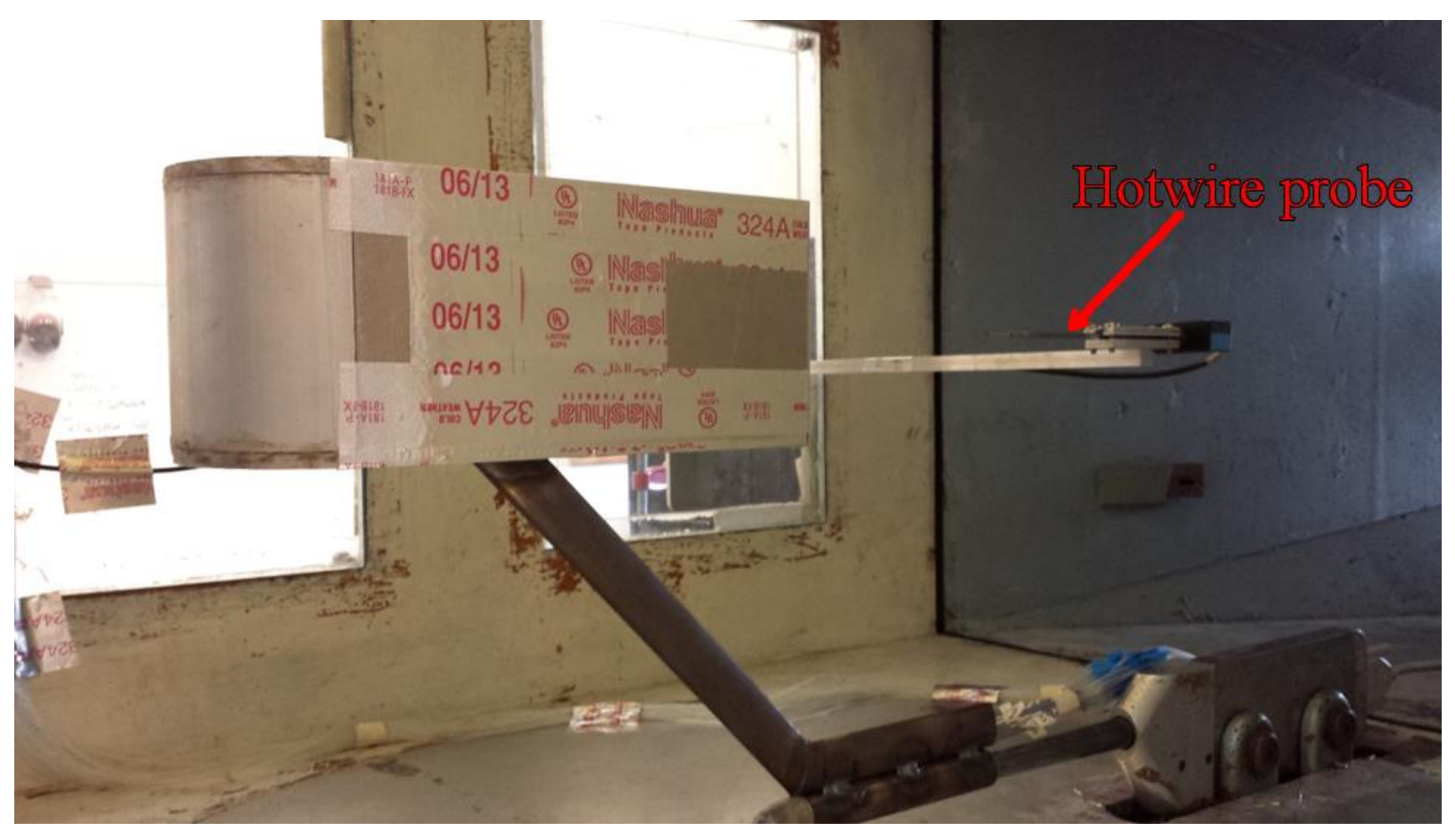

Fig. 17. Hotwire probe behind model.

The probe that was used in this experiment was a TSI model 1247A-T1.5 as seen in

Fig. 18. This probe was a two-channel probe but one wire was broken making it a onechannel probe. This probe only had a working channel two wire, which had a recommended operating resistance of $12.12 \Omega$ and an operating temperature of $250^{\circ} \mathrm{C}$. 


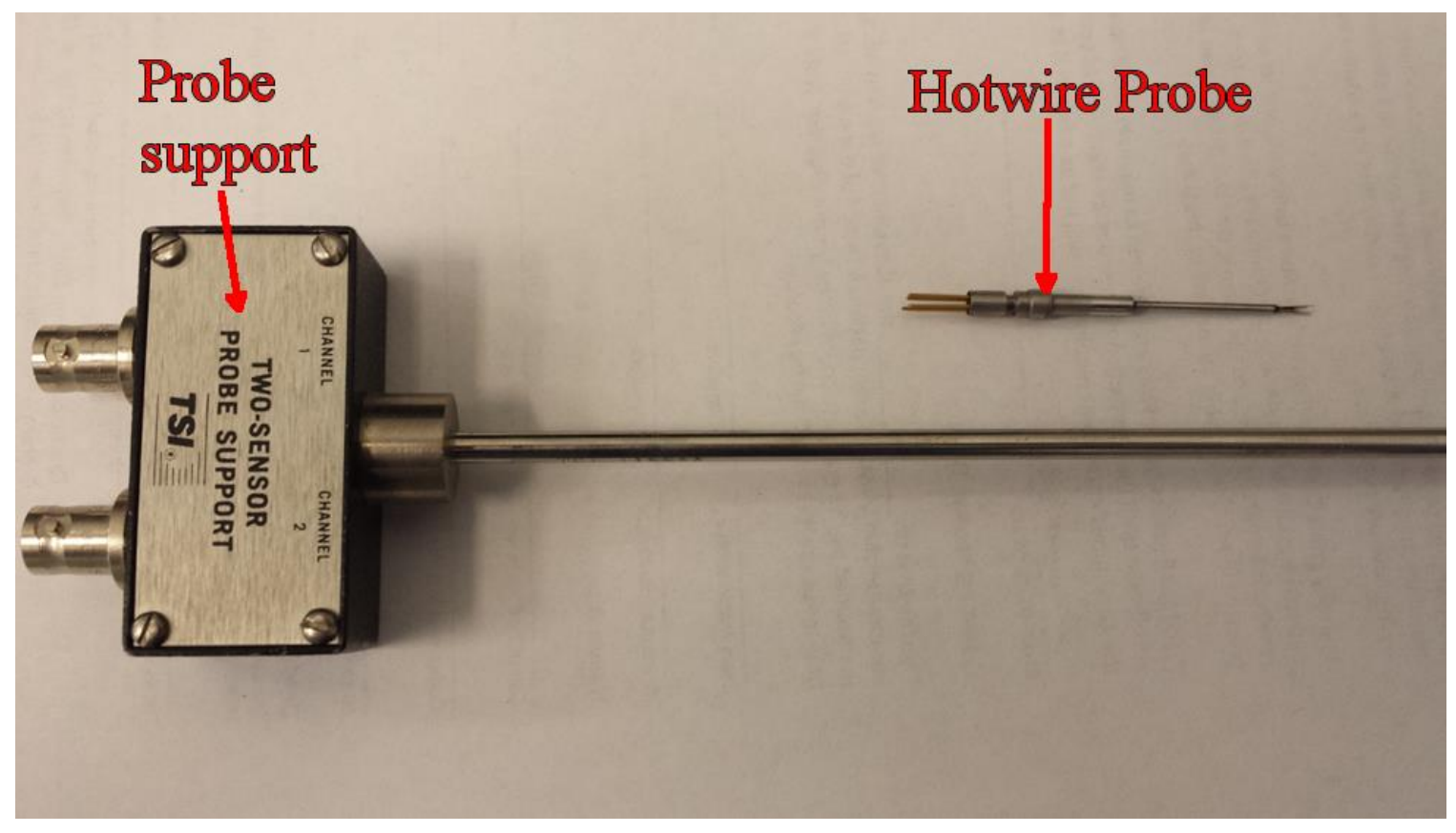

Fig. 18. Hotwire probe and probe support.

The hotwire was calibrated using the TSI model 1128A manual velocity calibrator in Fig. 19 along with a 0 to $10 \mathrm{mmH}_{\mathrm{g}}$ pressure transducer in Fig. 16 and the calibration manual [9]. The calibrator has a rotating probe mount. In this experiment the probe support was positioned to be directly above and parallel to the calibration flow source. The $448 \mathrm{kPa}(65 \mathrm{psi})$ compressed air in the lab was run through a pressure regulator that limits the pressure between $137.89 \mathrm{kPa}(20 \mathrm{psi})$ and $206.84 \mathrm{kPa}$ (30psi). This was then connected to the bottom of the calibrator. Coarse and fine adjustment knobs were used to accurately control the flow velocity coming out of the calibrator. The pressure of the flow was then sent to the pressure transducer and compared to the ambient pressure. This pressure difference and the voltage read from the hotwire probe were sent into the computer and analyzed with ThermalPro to create a relationship between voltage and velocity. 


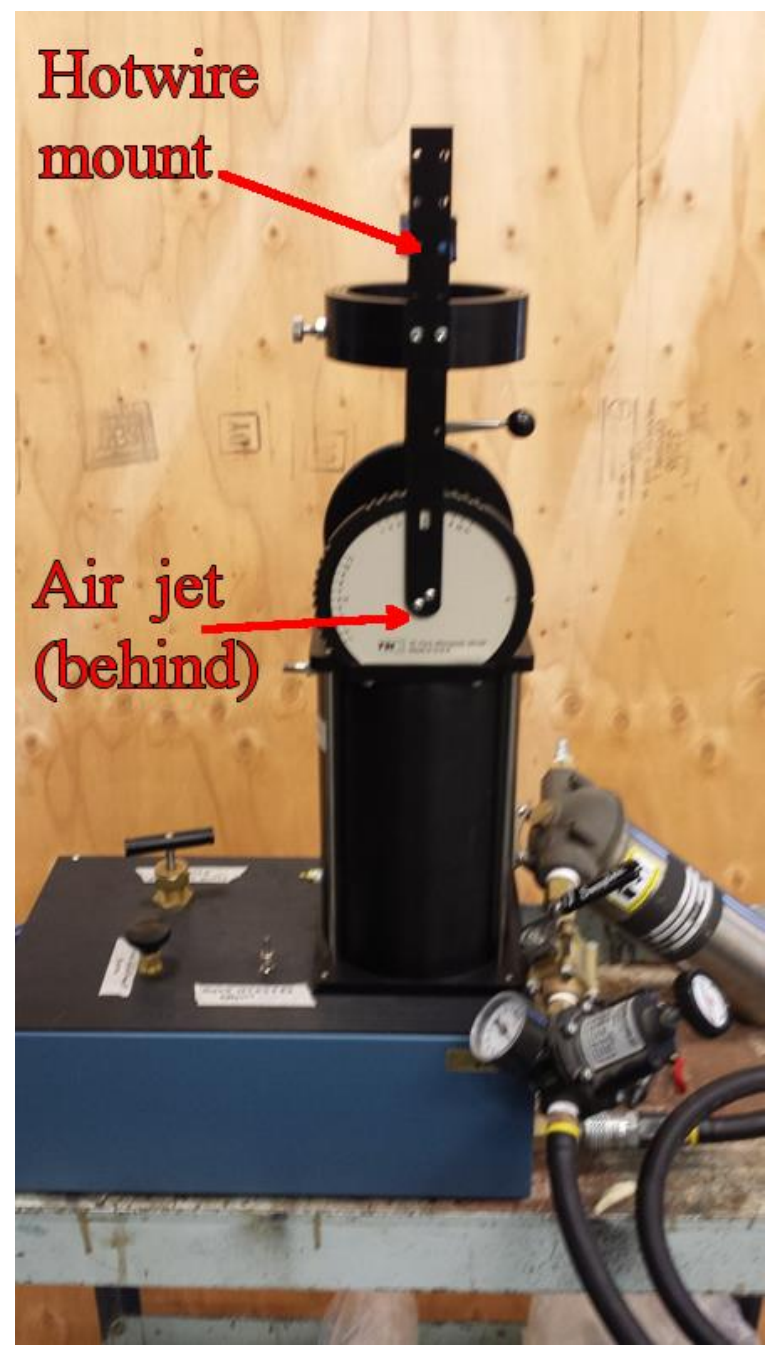

Fig. 19. Manual hotwire velocity calibrator. 


\section{PROCEDURES}

This section goes into the details for the procedure for each test. These tests include wind tunnel calibration, longitudinal pressure profile, boundary layer profile, base pressure, drag force, and hotwire velocity fluctuations.

\subsection{Wind Tunnel Calibration}

To calibrate the wind tunnel, a curve comparing the frequency of the motor to the velocity of the wind tunnel was created. This linear curve can be seen in the results section. To construct this curve, the dynamic pressure was measured at various wind tunnel VFD frequencies. The wind tunnel VFD controller in Fig. 20 was able to accept manual frequency inputs and hold them to $\pm 1 \mathrm{~Hz}$. The dynamic pressure of the tunnel and hence the wind tunnel velocity was measured using the static rings built into the inlet of the wind tunnel. The free stream dynamic pressure of the model was determined by a local Pitot-static tube. A correction factor was then calculated between the dynamic pressure at the inlet and the dynamic pressure at the test section. Using this correction factor and the known VFD frequencies for inlet dynamic pressure, an equation was created comparing the velocity in the test section to the VFD frequency. 


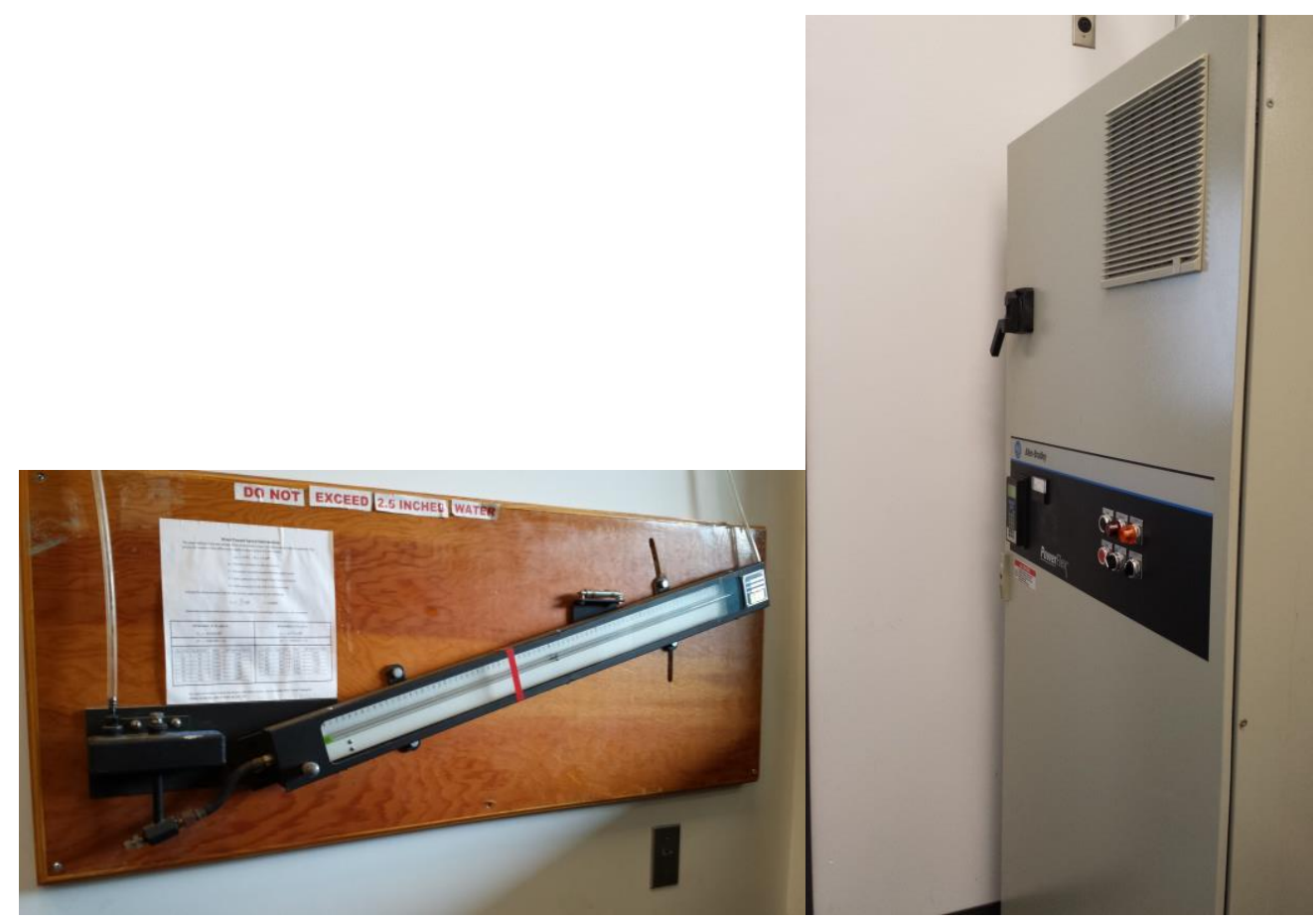

Fig. 20. Wind tunnel Variable Frequency Drive controller (right) and dynamic pressure anemometer (left).

\subsection{Longitudinal Pressure Profile}

Before any experimental testing could begin, the pressure profile from the nose of the model to the trailing edge of the model analyzed to ensure the profile matched previous work and to show that the model was aligned in the flow.

This test was conducted separately from the rear plate testing because only 30 pressure ports could be tested at a time due to the limited number of aluminum tubes which could pass through the strut. The wind tunnel was then run at all three speeds and data was taken for pressure ports 1 through 30 . The first 15 ports were along the centerline of the model that start at the leading edge and continue to the trailing edge with the last 15 ports representing the base region. There were also two data points taken for the free stream 
static pressure and total pressure that were taken from the Pitot-static probe in the test section. The data was recorded via RadLink and then processed with Matlab. A separate data file was created for each speed and each tab configuration. The most important profile test was the non-tabbed configuration because it shows the continuity of the flow over the model. The pressure profile was measured for the other three configurations to show changes in the base region.

\subsection{Boundary Layer Velocity Profile}

To measure the boundary layer, the probe was first put directly against the trailing edge of the model. A data point was then taken and the boundary layer probe was moved further away from the model incrementally taking new data points until the measured total pressure matched the free stream total pressure. Each time the pressure was measured with the boundary layer probe, the pressure was also measured in the model's static port to calculate the velocity. Due to stepping motor problems the screw on the traverse was turned manually to move the pressure probe. Each data point in the boundary layer was taken at an additional $1 / 8^{\text {th }}$ turn of the screw which correlates to $0.0003 \mathrm{~m}\left(1 / 80^{\text {th }}\right.$ of an inch) away from the previous point. The dynamic pressure at each point was then converted to a velocity using the total pressure from the boundary layer probe and the pressure from the static port on the model. The velocity profile was then analyzed using Matlab and the momentum thickness was calculated. The momentum thickness was then compared with previous experiments to calculate the optimal tab heights for each speed. 


\subsection{Base Pressure}

The base pressure was measured for all three speeds in each of the no tab and three tabbed configurations. This resulted in a total of twelve different test cases. Due to the lack of space in the strut mount, only thirty ports could be tested at a time. For the first round of testing the normal row of ports behind the tabs was tested along with the spanwise ports. The second round of testing measured the normal row behind the tabs as well as the normal row between the tabs. Each tab configuration was tested at all three speeds, creating a data file for each speed. After all three speeds were tested the tab configuration was changed and the three speeds were tested again. Changing the tab configuration was done without opening the model via swapping the removable back plates. When the model was mounted in the wind tunnel, the normal row was oriented horizontally with respect to the wind tunnel and the spanwise row was oriented vertically with respect to the tunnel. In this orientation the tabs are on the left and right sides of the model. The pressure data was read from each data file and converted to $\mathrm{C}_{\mathrm{Pb}}$ data and analyzed in Matlab.

\subsection{Sting Balance}

The sting balance data was taken using LabView and processed using Excel and Matlab. Before the testing began, the sting balance was calibrated using the correlation between the measured voltage in the strain gage and the known calibration weights. A baseline was then taken with the model mounted to the sting balance without any flow. This allowed the model weight to be removed from the equation and only the effects of the flow on the model were shown in the results. To this same end, the tunnel was run at all three test speeds with only the strut mounted to the sting so its effects could also be 
removed from the results. Total force measurements were then taken at all three speeds for all four tab configurations. The force on the body was then calculated by subtracting the force caused by the weight of the model as well as the force caused by the strut mount from the total force measurements. Next the Coefficient of Drag was calculated using Equ. (3).

\subsection{Hotwire Velocity Spectra}

After the hotwire was successfully calibrated the hotwire probe and probe support were removed from the calibrator. The probe was then removed from the probe support for safe keeping. Next, the probe support was installed on the end of the traverse and moved into position $0.0254 \mathrm{~m}$ ( 1 inch) from the trailing edge of the model. The probe was aligned parallel with one side of the model directly behind the center tab. The hotwire probe was then reinserted into the probe support. Spectral density data was then taken using ThermalPro for all three test speeds and all four tab configurations. For all four tests and all three speeds the data was taken at $1 \mathrm{kHz}$ with a sample size of $16 \mathrm{kpts}$. This results in a frequency resolution of $0.977 \mathrm{~Hz}$. Between each test configuration the probe was removed while the tabbed plates were swapped to ensure the wire stayed intact. The spectral density data was then processed in Matlab to calculate the energy spectrum and Strouhal numbers. Calibration of the hotwire is discussed in more detail in the results section. 


\section{ANALYSIS}

This section is meant as a brief reference describing the major coefficients and nondimensional numbers in this paper and how they are calculated.

\subsection{Reynolds Number}

The Reynolds number is a dimensionless number that gives a ratio of inertial forces to viscous forces. Normally the Reynolds number is calculated based on the length of the model but in this experiment the Reynolds number is calculated based on model height to stay consistent with previous experiments.

$$
R e_{h}=\frac{u_{\infty} * h}{v}
$$

Where $h$ is the height of the model, $u_{\infty}$ is the free stream velocity, and $v$ is the kinematic viscosity. This equation gives Reynolds numbers of $8.3 \mathrm{e} 4,1.6 \mathrm{e} 5$ and $2.5 \mathrm{e} 5$ for the test speeds of $10 \mathrm{~m} / \mathrm{s}, 20 \mathrm{~m} / \mathrm{s}$, and $30 \mathrm{~m} / \mathrm{s}$ respectively. A standard kinematic viscosity of $1.52 \mathrm{e}-5 \mathrm{~m}^{2} / \mathrm{s}$ was used as the standard is close to the experimental conditions in San Luis Obispo.

\subsection{Pressure Coefficient}

The pressure coefficient is a dimensionless number that compares the pressures throughout the flow field. In this experiment the pressure coefficient is calculated along the longitudinal profile of the body and the base region to show the pressure distribution around the body. The pressure coefficient is calculated by:

$$
C_{P}=\frac{P-P_{\infty}}{q_{\infty}}=\frac{P-P_{\infty}}{P_{T}-P_{\infty}}
$$

Where $P$ is the pressure measured at each static port on the model, $q_{\infty}$ is the free stream dynamic pressure which is calculated from the free stream static pressure, $P_{\infty}$, and 
the free stream total pressure, $P_{T}$. An example of the propagation of error in the pressure coefficient can be seen in Appendix A.

\subsection{Drag Coefficient}

The drag coefficient is a non-dimensional quantity that is used to compare the drag forces across different speeds of the experiment. The drag coefficient is calculated by:

$$
C_{D}=\frac{D}{q_{\infty} * S}
$$

where $D$ is the drag force on the body, $q_{\infty}$ is the free stream dynamic pressure, and $S$ is the cross sectional area of the body $(h * h)$. An example of the propagation of error in the drag coefficient can be seen in Appendix A.

\subsection{Strouhal Number}

The Strouhal number is a ratio that describes the oscillations in a flow. This number is useful in hotwire testing because it allows for comparison of frequencies between tests with inputs of only model height and free stream velocity. This is calculated using this equation:

$$
S t=\frac{f * h}{u_{\infty}}
$$

where $\mathrm{f}$ is the vortex shedding frequency, $\mathrm{h}$ is the model height, and $u_{\infty}$ is the free stream velocity. 


\section{RESULTS AND DISCUSSION}

Here the results are shown and analyzed for each of the five tests performed. The discussion in this section is meant to interpret the results individually and the reasoning for why the results came out this way will be looked at in the following discussion section.

\subsection{Wind Tunnel Correlation Results}

To create the wind tunnel correlation trend seen in the typical example of Fig. 21, the frequency was set at increments of $5 \mathrm{~Hz}$ from $10 \mathrm{~Hz}$ to $40 \mathrm{~Hz}$ and a final point was added for $0 \mathrm{~Hz}$. For this example the dynamic pressure was measured using a Pitot-static probe in the tunnel test section without the model present. The average temperature in San Luis Obispo during testing was $21^{\circ} \mathrm{C}\left(70^{\circ} \mathrm{F}\right)$ so a standard density of $1.292 \mathrm{~km} / \mathrm{m}^{3}(1.225$ slugs $/ \mathrm{ft}^{3}$ ) and Equ. (5) below, the calibration curve shown in Fig. 21. 
Tunnel Speed Calibration

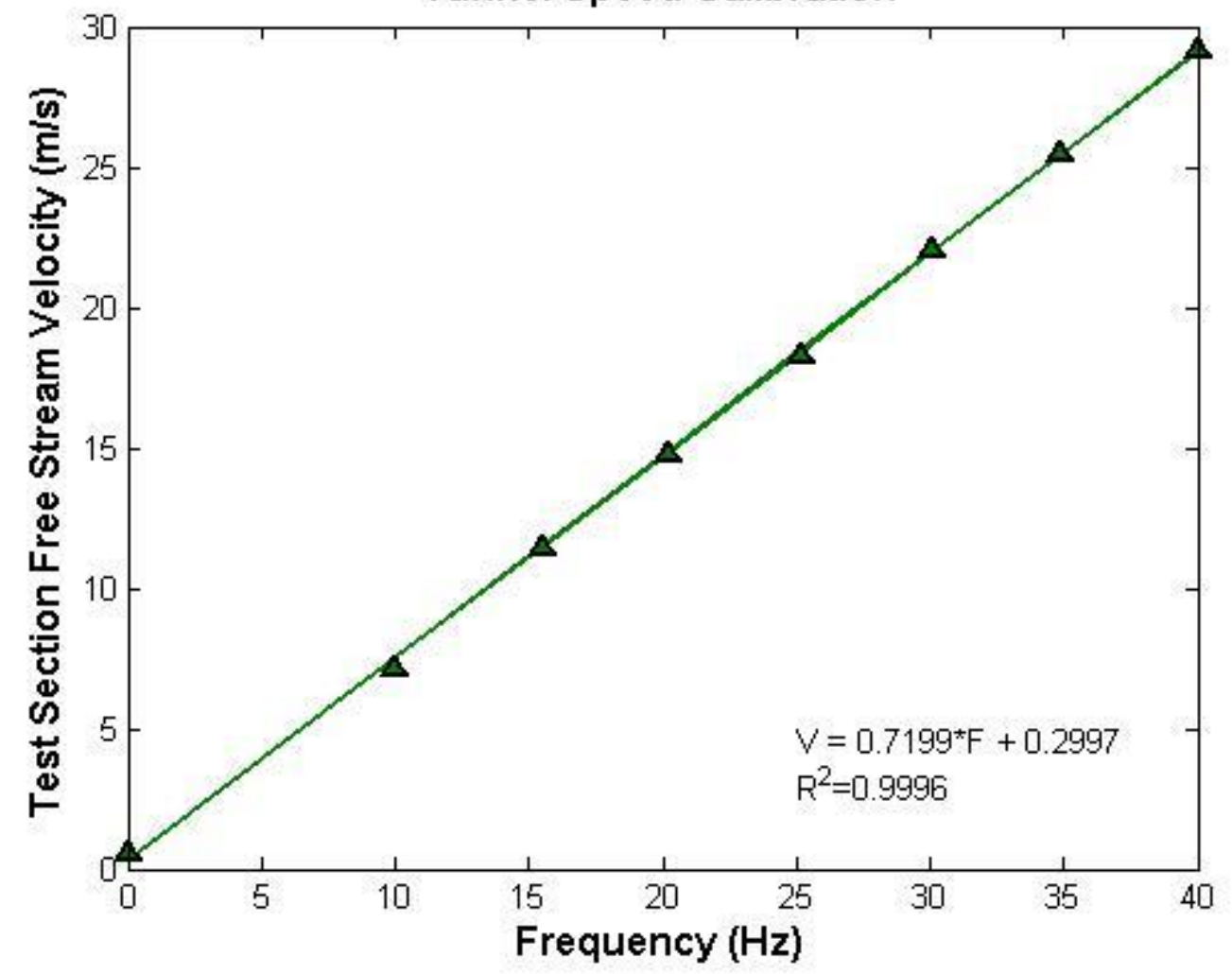

Fig. 21. Wind tunnel calibration results.

$$
V=\sqrt{\frac{2 * q}{\rho}}
$$

where $q$ is the dynamic pressure and $\rho$ the ambient density.

The tunnel calibration tests yielded a linear relationship, which is the same as previous experiments [6]. Such a relationship allowed for more accurate speed measurements for the pressure profile and boundary layer testing by setting the frequency directly in the wind tunnel controller.

\subsection{Longitudinal Pressure Profile}

The first test on the model was the longitudinal pressure profile test. This test showed what was expected and verified results from previous works. As shown in Fig. 22, the first port is expected to have a $C_{P}$ of 1 as it shows the stagnation point on the nose of the 
model. The $C_{P}$ of the nose for this model is 0.998 , which is very close to 1 . This signifies that the model is aligned with the flow and the pressure transducer was working correctly. The next point of interest is the $5^{\text {th }}$ pressure port. This port is at the transition from the curved surface of the nose to the flat surface of the body. This point has the lowest $\mathrm{C}_{\mathrm{P}}$ because it is where the flow was most accelerated along the body. The final point of interest is where the flow transitions to the base region at port 15 . This test needed to show that without tabs, the pressure was continuous from the attached flow into the base region. This continuity shows that the model was built correctly and aligned properly in the flow as well as to show that the pressure transducer was reading the pressure accurately. The results from this experiment were compared with Fig. 23 from Knight and Tso's experiment [4] below to show similarities in the results.

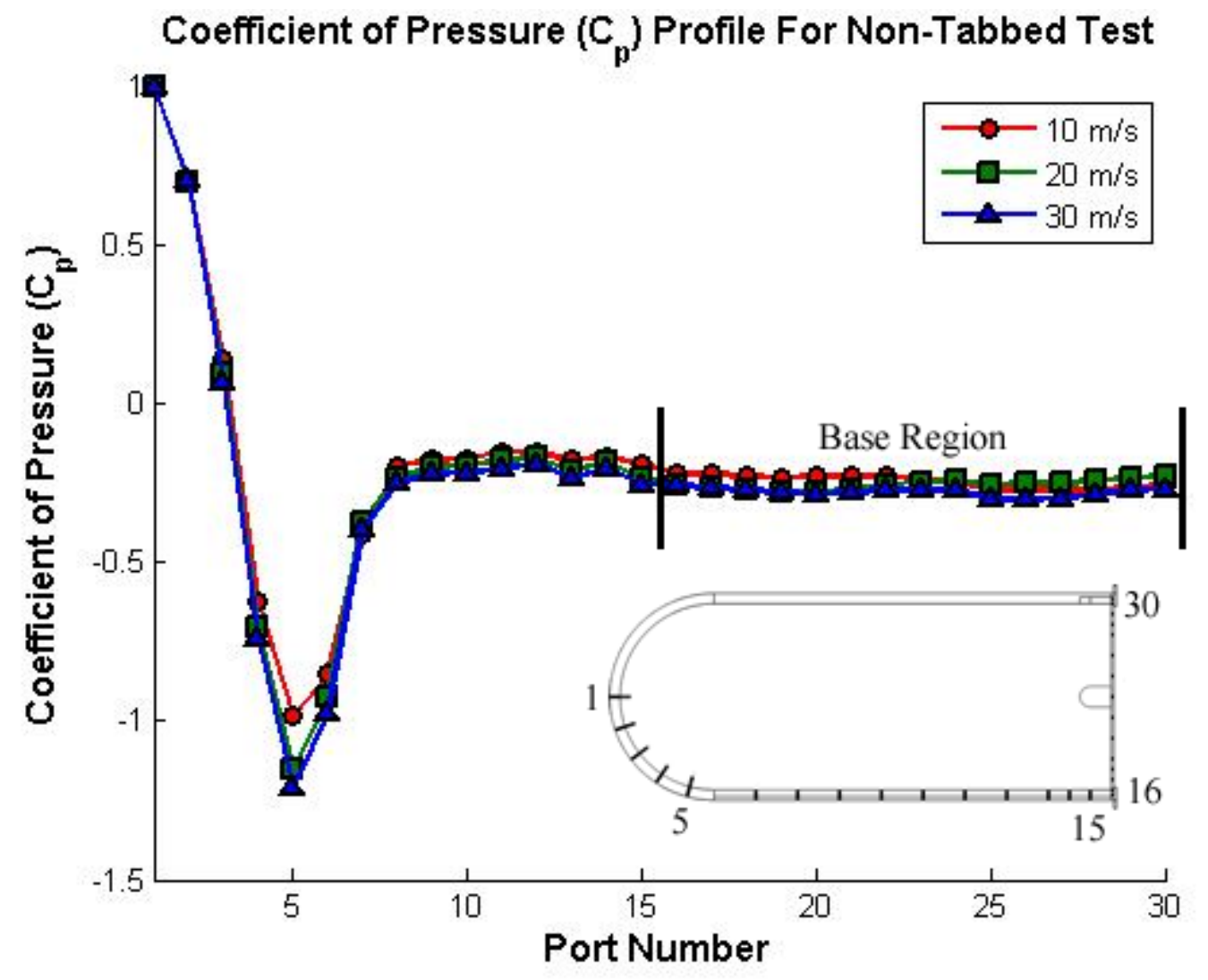

Fig. 22. This experiment's pressure coefficient profile results. 


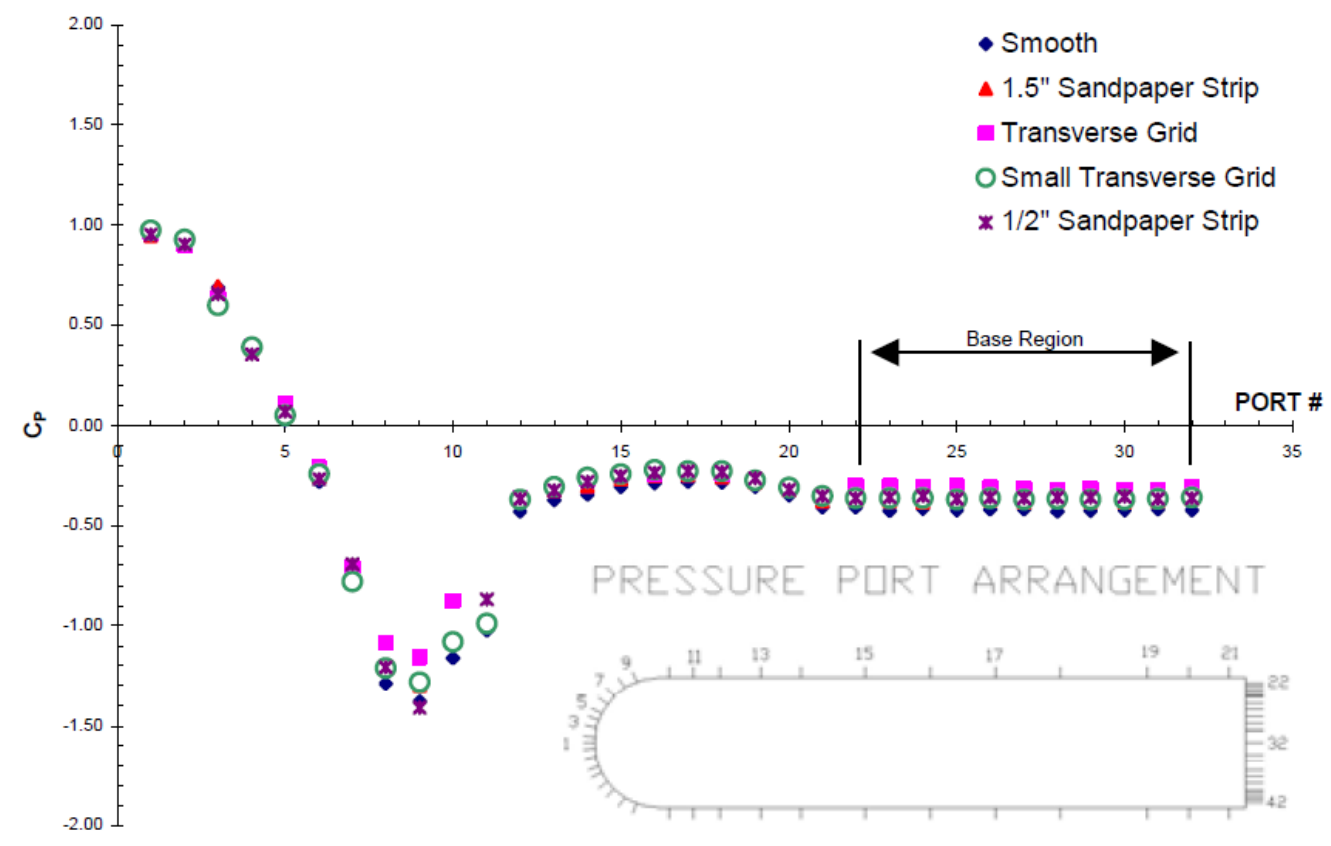

Fig. 23. Knight and Tso's pressure profile results [4].

\subsection{Boundary Layer Velocity Profiles}

The second test for this experiment was the boundary layer measurements. These measurements were necessary to determine the optimal tab height for each speed. The pressure data was averaged over two minutes at each data point to reduce inconsistencies from unsteady pressure. After each data point was taken, the boundary layer probe was moved an additional $0.0003 \mathrm{~m}\left(1 / 80^{\text {th }}\right.$ of an inch) away from the model until the velocity profile became vertical.

Originally the data was interpreted visually, looking for where the curve became vertical to find the boundary layer thickness. This analysis turned out to be too subjective and instead the velocity profile was used to calculate the momentum thickness from Equ. 6. The momentum thickness was then used to find optimal tab height. 


$$
\theta=\int_{0}^{\delta} \frac{u}{U_{\infty}}\left(1-\frac{u}{U_{\infty}}\right) d y
$$

Since this was a finite data set, a $d y$ of $0.0003 \mathrm{~m}$ (1/80inches) was used in the trapezoidal integral approximation in MATLAB. The results from this test correlated well with the Knight and Tso's results [4]. However, to calculate the tab heights a scaling factor had to be applied to the measured momentum thickness. This scaling factor was found by comparing Knight and Tso's boundary layer data [4] to Pinn's tab height data [6] and finding a correlation factor of about three. This factor of three was then applied to the measured momentum thickness to obtain the tab heights. The calculated tab heights are $0.0059 \mathrm{~m}, 0.0043 \mathrm{~m}$, and $0.0038 \mathrm{~m}(0.2338,0.1687$, and 0.1487 inches $)$ for the $10 \mathrm{~m} / \mathrm{s}$, $20 \mathrm{~m} / \mathrm{s}$, and $30 \mathrm{~m} / \mathrm{s}$ speeds respectively. The results can be seen in Table 1 below. To calculate the boundary layer height a trend line was applied to the velocity profile and the thickness was found where $\mathrm{u} / \mathrm{U}_{\infty}>0.995$. These results compared well with Knight and Tso's [4] results with the same trip tape. The results also show a decreasing boundary layer with increasing speed, which is expected from the theory.

Table 1. Boundary Layer Results

\begin{tabular}{|c|c|c|c|c|}
\hline Speed & Momentum & Boundary Layer & Correlation & Tab Height \\
& Thickness & Thickness & Factor & \\
\hline $10 \mathrm{~m} / \mathrm{s}$ & $0.0019 \mathrm{~m}$ & $0.0183 \mathrm{~m}$ & 3.03 & $0.0059 \mathrm{~m}$ \\
$(32.8 \mathrm{ft} / \mathrm{s})$ & $(0.0771 \mathrm{in})$ & $(0.7205 \mathrm{in})$ & $(0.2338 \mathrm{in})$ \\
\hline $20 \mathrm{~m} / \mathrm{s}$ & $0.0011 \mathrm{~m}$ & $0.0101 \mathrm{~m}$ & 3.76 & $0.0043 \mathrm{~m}$ \\
$(65.6 \mathrm{ft} / \mathrm{s})$ & $(0.0449 \mathrm{in})$ & $(0.3995 \mathrm{in})$ & & $(0.1687 \mathrm{in})$ \\
\hline $30 \mathrm{~m} / \mathrm{s}$ & $0.0009 \mathrm{~m}$ & $0.0095 \mathrm{~m}$ & 3.80 & $0.0038 \mathrm{~m}$ \\
$(98.4 \mathrm{ft} / \mathrm{s})$ & $(0.0392 \mathrm{in})$ & $(0.3746 \mathrm{in})$ & & $(0.1487 \mathrm{in})$ \\
\hline
\end{tabular}




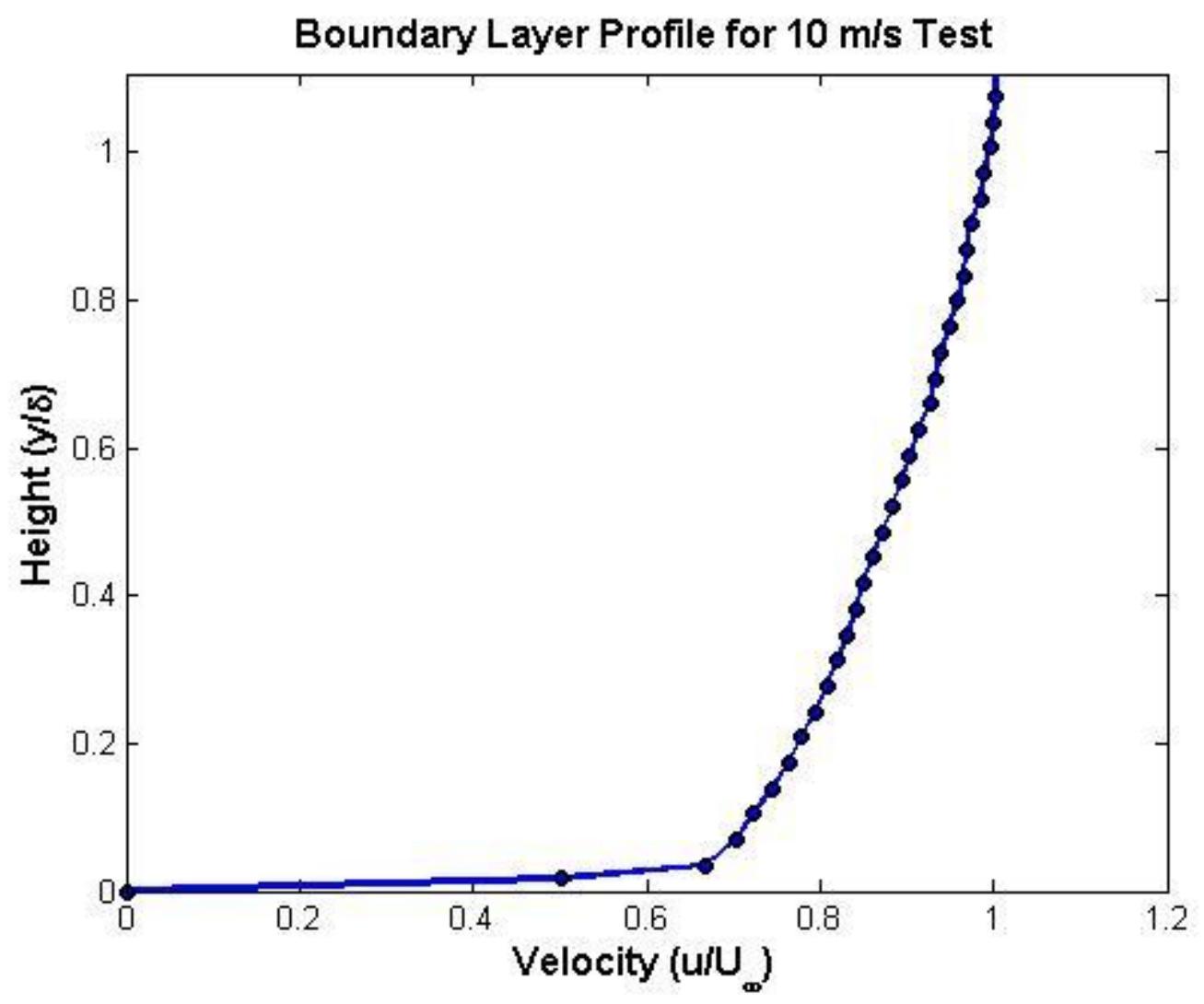

Fig. 24. Boundary layer velocity profile for $10 \mathrm{~m} / \mathrm{s}$ test.

Fig. 24 shows the test results for the boundary layer from the $10 \mathrm{~m} / \mathrm{s}$ test. This test had 32 points that lie within the boundary layer. Though more were taken to ensure the complete profile was captured. The point at zero was not a measured data point and was an extrapolation to preserve the no slip condition at the wall. The height of the first point was the distance from the edge of the model to the midpoint of the pressure probe. Every successive point was $0.0003 \mathrm{~m}\left(1 / 80^{\text {th }}\right.$ inches $)$ away from the previous point. This test resulted in a boundary layer thickness of $0.0183 \mathrm{~m}$ (0.7205 inches). 


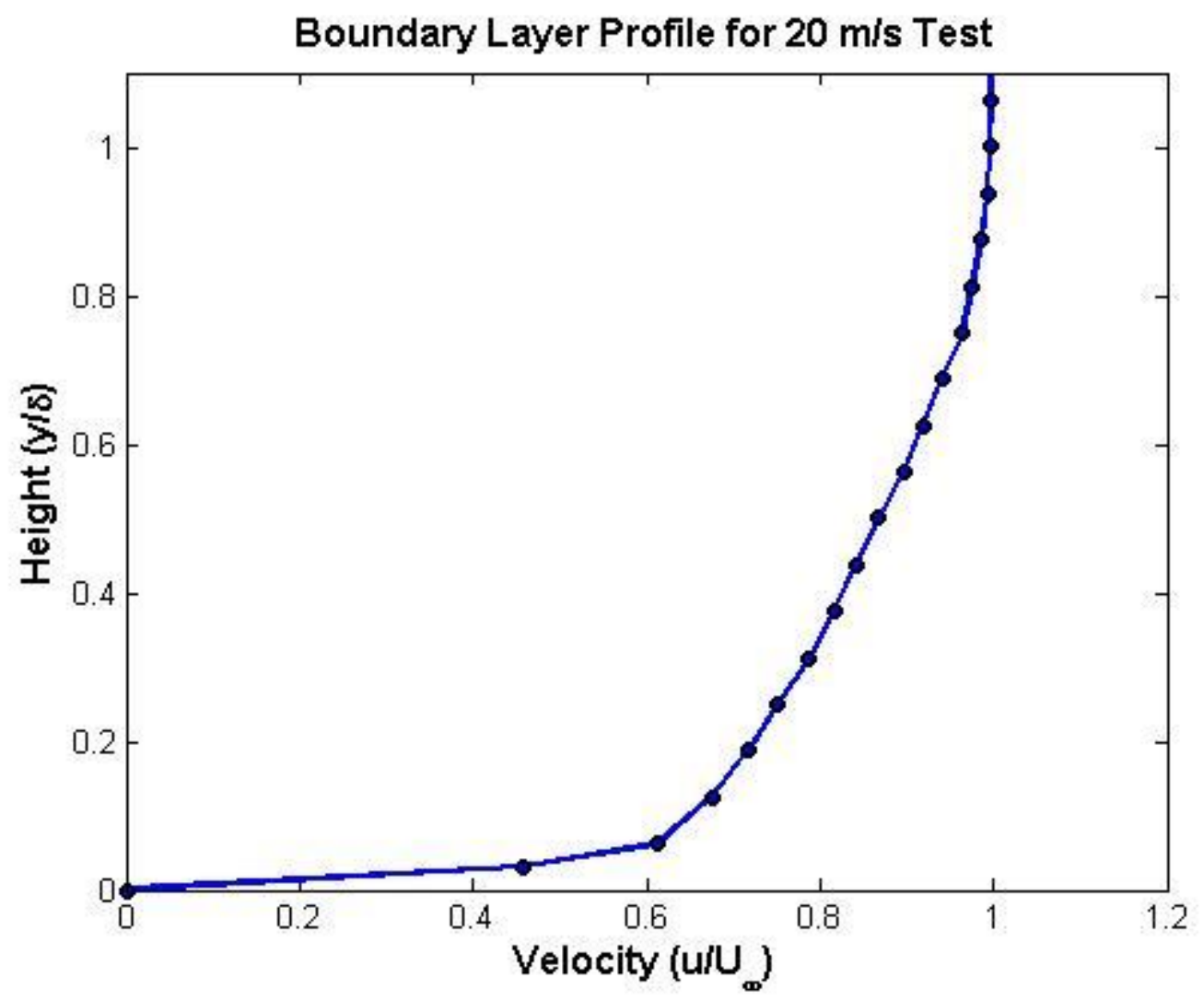

Fig. 25. Boundary layer velocity profile for $20 \mathrm{~m} / \mathrm{s}$ test.

The curve above shows the test results for the boundary layer from the $20 \mathrm{~m} / \mathrm{s}$ test. As with the $10 \mathrm{~m} / \mathrm{s}$ test, Fig. 25 only shows the 20 points that lie within the boundary layer. The point at zero was not a measured data point and was an extrapolation to preserve the no slip condition at the wall. The height of the first point was the distance from the edge of the model to the midpoint of the pressure probe. Every successive point was $0.0003 \mathrm{~m}$ $\left(1 / 80^{\text {th }}\right.$ inches) away from the previous point. This test resulted in a boundary layer thickness of $0.0101 \mathrm{~m}(0.3995$ inches $)$. 


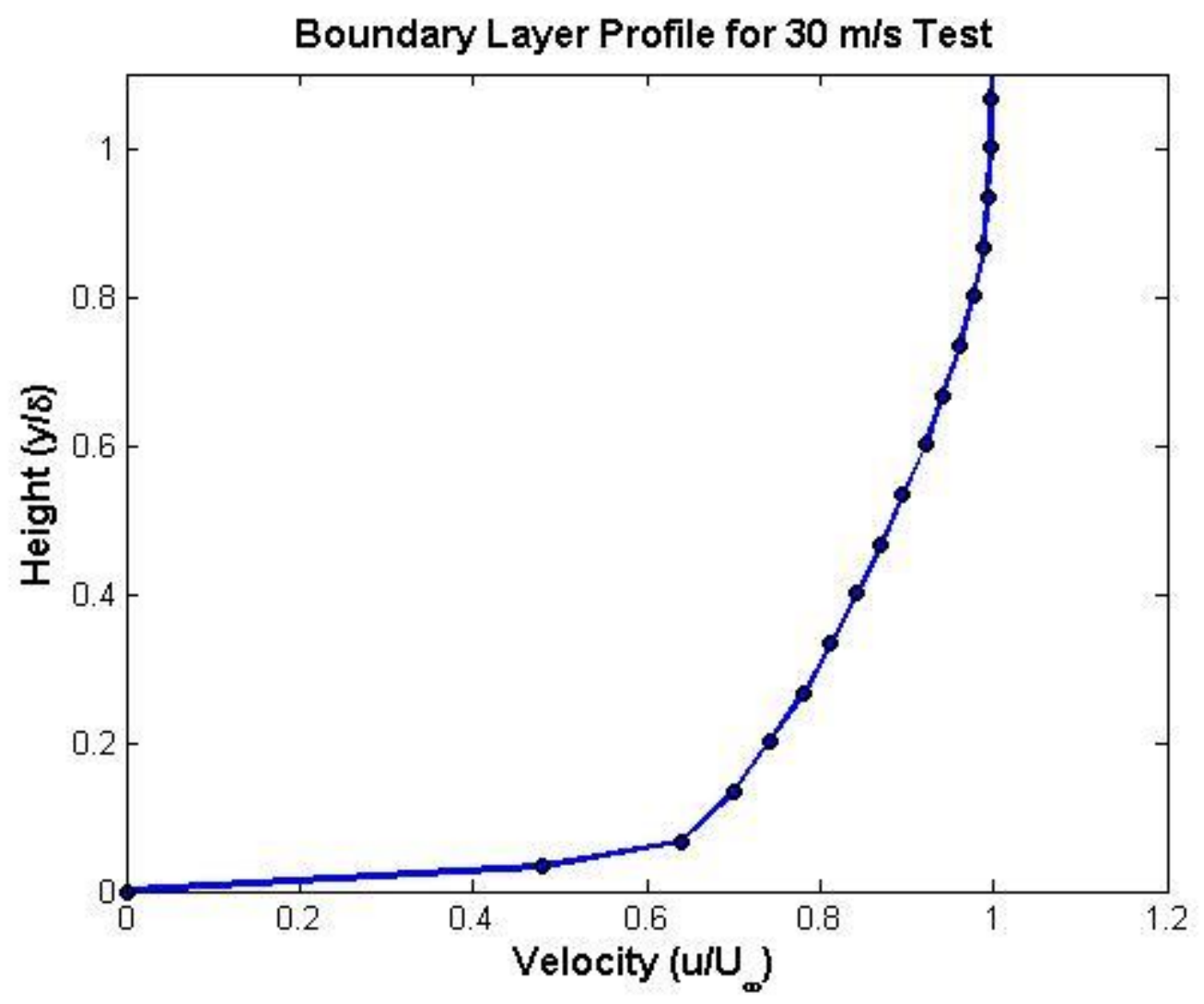

Fig. 26. Boundary Layer velocity profile for $30 \mathrm{~m} / \mathrm{s}$ testing.

Fig. 26 shows the test results for the boundary layer from the $30 \mathrm{~m} / \mathrm{s}$ test. This test has 18 points that lie within the boundary layer. Though more were taken to ensure the complete profile was measured. The point at zero was not a measured data point and was an extrapolation to preserve the no slip condition at the wall. The height of the first point was the distance from the edge of the model to the midpoint of the pressure probe. Every successive point was $0.0003 \mathrm{~m}\left(1 / 80^{\text {th }}\right.$ inches $)$ away from the previous point. This test resulted in a boundary layer thickness of $0.0095 \mathrm{~m}$ (0.3746 in).

\subsection{Base Pressure Coefficients}

To see the effects of the tabs on the base pressure of the model, the normal and spanwise rows of pressure ports were measured simultaneously. For each test configuration and test speed, pressure measurements were taken continuously for 3 
minutes and then averaged. This was done to remove fluctuations due to the turbulent flow. The data was then processed with Equ. (7).

$$
C_{P_{b}}=\frac{P-P_{\infty}}{K_{\varepsilon}\left(P_{t o t}-P_{\infty}\right)}
$$

where $P_{\infty}$ and $P_{\text {tot }}$ are the free stream and total pressures measured in the test section. $P$ is the pressure measured at the current pressure port, and $K_{\varepsilon}$ is the blockage coefficient due to the model in the flow calculated in Equ. (8-9).

$$
\varepsilon_{s b}=\frac{K v}{C^{3 / 2}}
$$

where $K$ is body shape factor taken from pg. 290 of Pope [10], $v$ is the volume of the model and $C$ is the area of the wind tunnel. This results in a $\varepsilon_{s b}$ of 0.0037 . The final blockage coefficient was calculated from Equ. (9) for a final value of $K_{\varepsilon}=1.0037$ :

$$
K_{\varepsilon}=1+\varepsilon_{s b}
$$




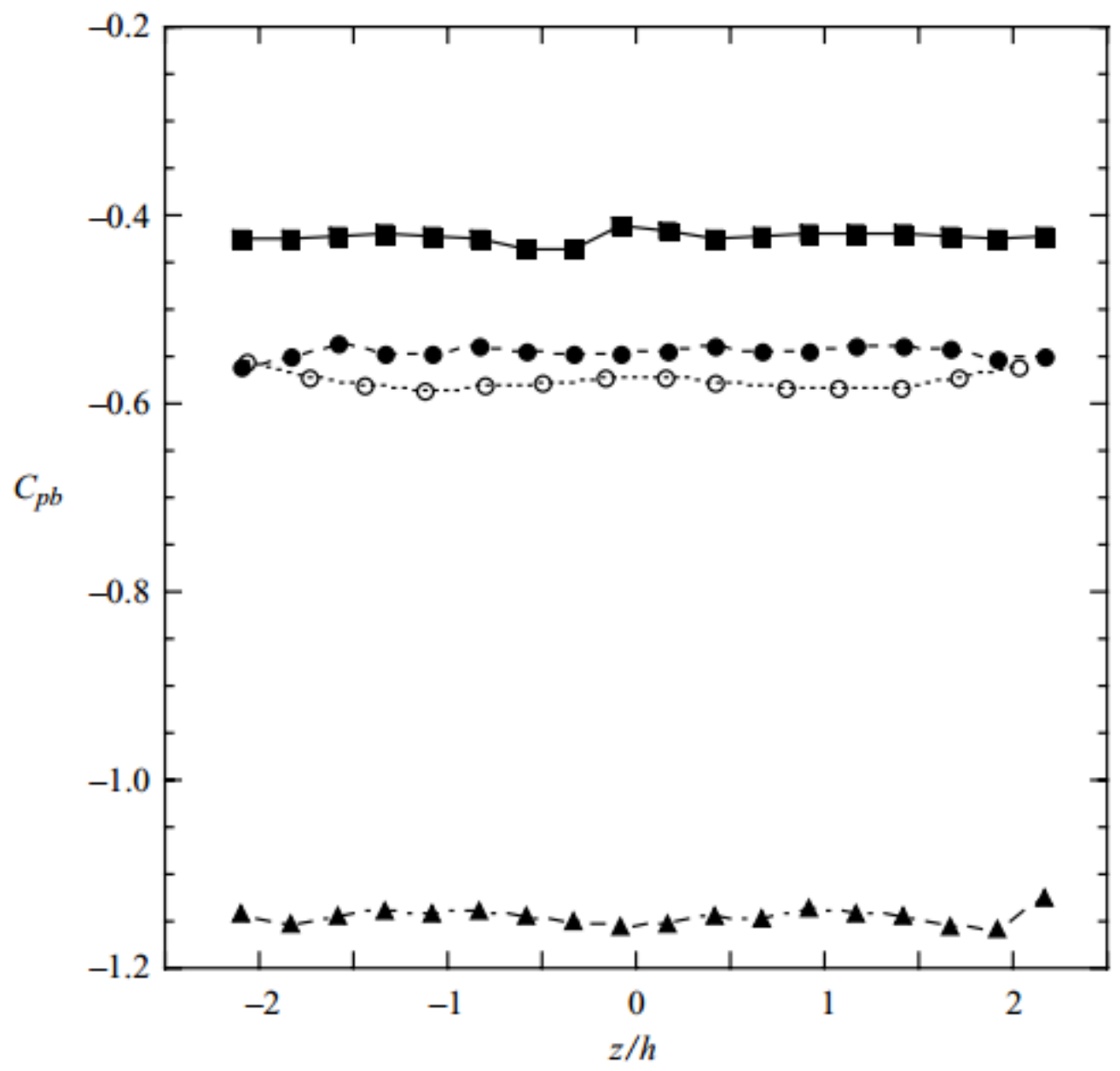

Fig. 27. Park et al. data [3]. Solid square was optimally controlled flow with a pair of tabs. Solid circle data was uncontrolled flow. Open circle data was Bearman's uncontrolled flow. Solid triangle data was a two dimensional fence of tabs.

Fig. 27 shows Park's et al. data [3] with controlled and uncontrolled tabs along with Bearman's data [1]. The first important trend to notice is both Bearman's and Park's et al. uncontrolled data was around the same mean $\mathrm{C}_{\mathrm{pb}}$. The next trend is that Bearman's high aspect ratio body has noticeable valleys in $\mathrm{C}_{\mathrm{pb}}$ data. These valleys showed the existence of vortex shedding. The next important trend was the solid square line which shows the optimally controlled flow. This trend shows near flat data indicating no vortex shedding. The controlled data also shows an increase in $\mathrm{C}_{\mathrm{pb}}$ which correlates to a drag reduction. 


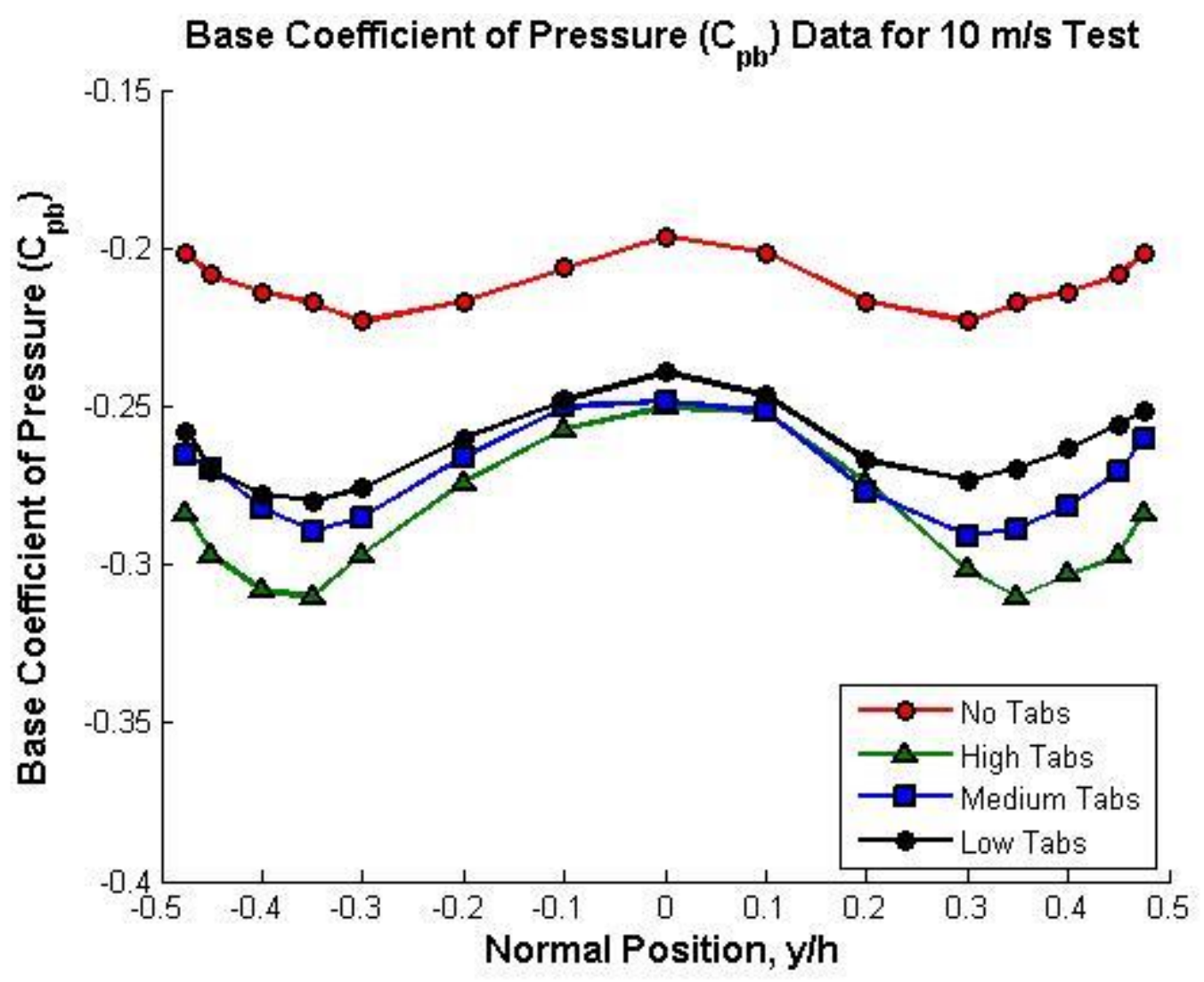

Fig. 28. Base pressure results for $10 \mathrm{~m} / \mathrm{s}$ tests.

Fig. 28 shows the base pressure results in the normal position on the centerline of the model for the $10 \mathrm{~m} / \mathrm{s}$ case. These results showed very similar results to Park et al. [3] and Bearman's results [1,2] for the uncontrolled case. The high aspect ratio experiments were able to show that there were two distinct valleys in the pressure data with no flow control devices. Though the non-tabbed data was consistent with previous results, the tabbed data from this experiment differed greatly from previous controlled flow data. In Park et al. [3] results the controlled tests showed a flat base pressure curve with increased average $\mathrm{C}_{\mathrm{pb}}$. The tabbed case in this experiment showed just the opposite. In this experiment the tabs showed a decrease in average $\mathrm{C}_{\mathrm{pb}}$ and two much deeper valleys. Both of these trends suggest an increase in vortex strength instead of a reduction. As shown in Table 2, the 
high tabs had the largest negative impact on the $\mathrm{C}_{\mathrm{pb}}$ of the bluff body. This suggested that larger tabs cause a larger increase in drag than the other tab heights.

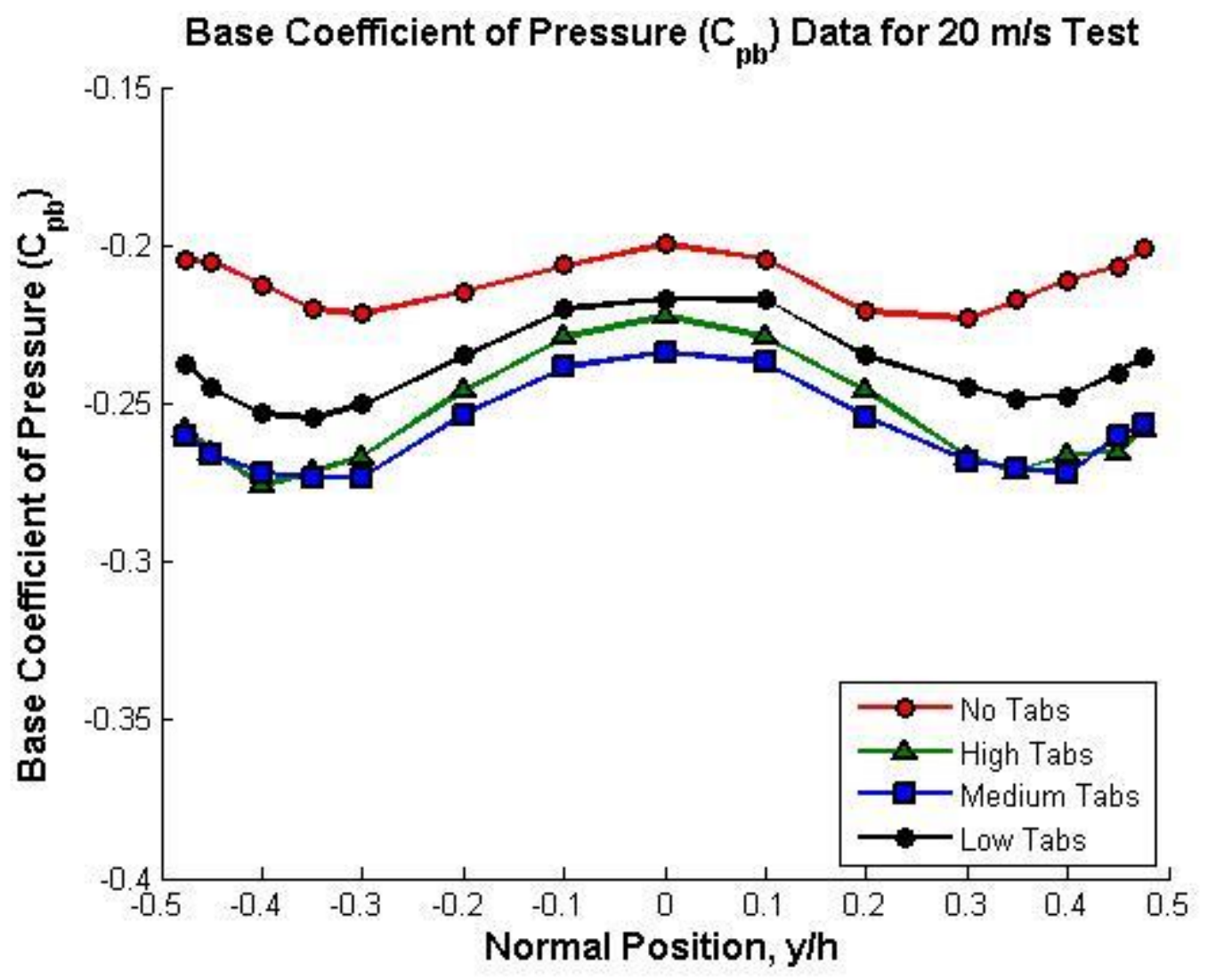

Fig. 29. Base Pressure results for $20 \mathrm{~m} / \mathrm{s}$ tests.

Fig. 29 shows the base pressure results in the normal position on the centerline of the model for the $20 \mathrm{~m} / \mathrm{s}$ case. The results above show similar results to the $10 \mathrm{~m} / \mathrm{s}$ tests. In this test it was shown that the non-tabbed case had the highest average $C_{p b}$ and had the shallowest valleys. These two results proved that the tabs increase the vortex strength and suggested an increase in the mean drag on the model for this speed as well as for $10 \mathrm{~m} / \mathrm{s}$. 


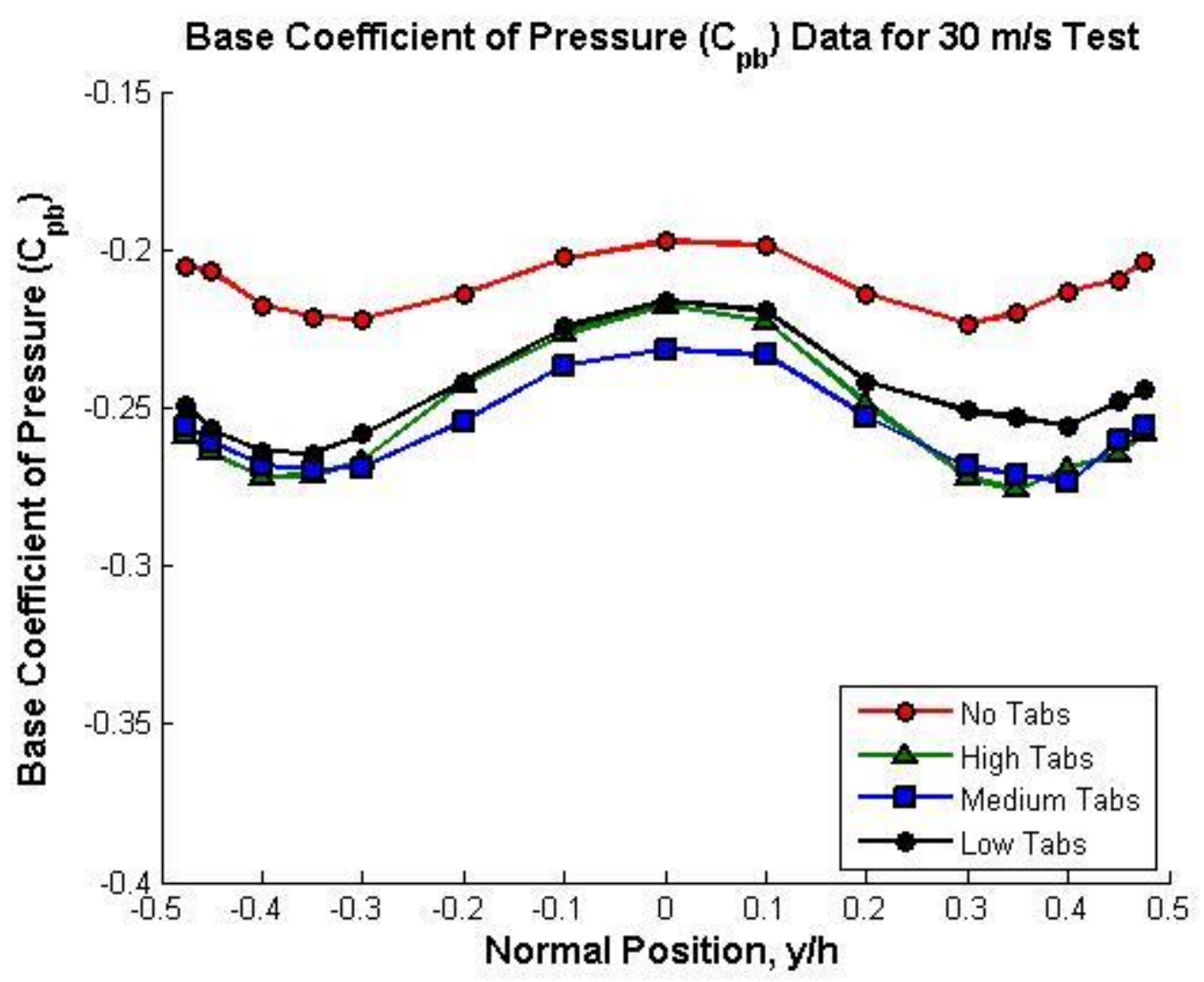

Fig. 30. Base pressure profiles for $30 \mathrm{~m} / \mathrm{s}$ tests.

Fig. 30 shows the base pressure results in the normal position on the centerline of the model for the $30 \mathrm{~m} / \mathrm{s}$ case. In this test it is shown that the non-tabbed case had the highest average $\mathrm{C}_{\mathrm{pb}}$ and had the shallowest valleys, while the medium and high tabs had roughly the same effects on $\mathrm{C}_{\mathrm{pb}}$ valley strength. These results proved that the tabs increased the vortex strength and suggest an increase in the mean drag on the model for all three speeds. As seen in Table 2 below, the low tabs showed the least amount of negative impact of the three tabbed configurations for all three test speeds. Looking at the trends in Table 2, it can be shown that the average $\mathrm{C}_{\mathrm{pb}}$ impacts decreased with decreasing tab heights for all three speeds.

The results from the tests above suggest that the drag increases with the addition of the tabs. Looking more closely at the base pressure curves, there exist a few noticeable 
trends. The first trend is that the curves have two valleys. These valleys show the existence of the Kármán Vortex shedding. With the addition of the tabs these valleys get deeper instead of shallower, which shows a stronger vortex. Finally the average base pressure of the model with the addition of the tabs is lower than the tests without the tabs. This difference also suggests an increase in base suction on the model and thus an increase in the mean drag of the model.

It is important to note that using the scale from Jarred Pinn's [6] and Park's et al. [3] results it would appear that there were no vortices shedding on any of the test cases. At that scale all the curves appear to be flat. Using the scale of Erlhoff's [8] tests and Bearman's [1,2] tests, the two valleys of $\mathrm{C}_{\mathrm{Pb}}$ data can be seen more clearly.

Table 2. Changes in average $C_{P b}$ Magnitude Compared to No Tab Configuration.

\begin{tabular}{|c|c|c|c|}
\hline & $10 \mathrm{~m} / \mathrm{s}$ & $20 \mathrm{~m} / \mathrm{s}$ & $30 \mathrm{~m} / \mathrm{s}$ \\
\hline No Tabs & 0 & 0 & 0 \\
\hline Low Tabs & -0.0448 & -0.0316 & -0.0345 \\
\hline Medium Tabs & -0.0446 & -0.0370 & -0.0370 \\
\hline High Tabs & -0.0604 & -0.0507 & -0.0468 \\
\hline
\end{tabular}



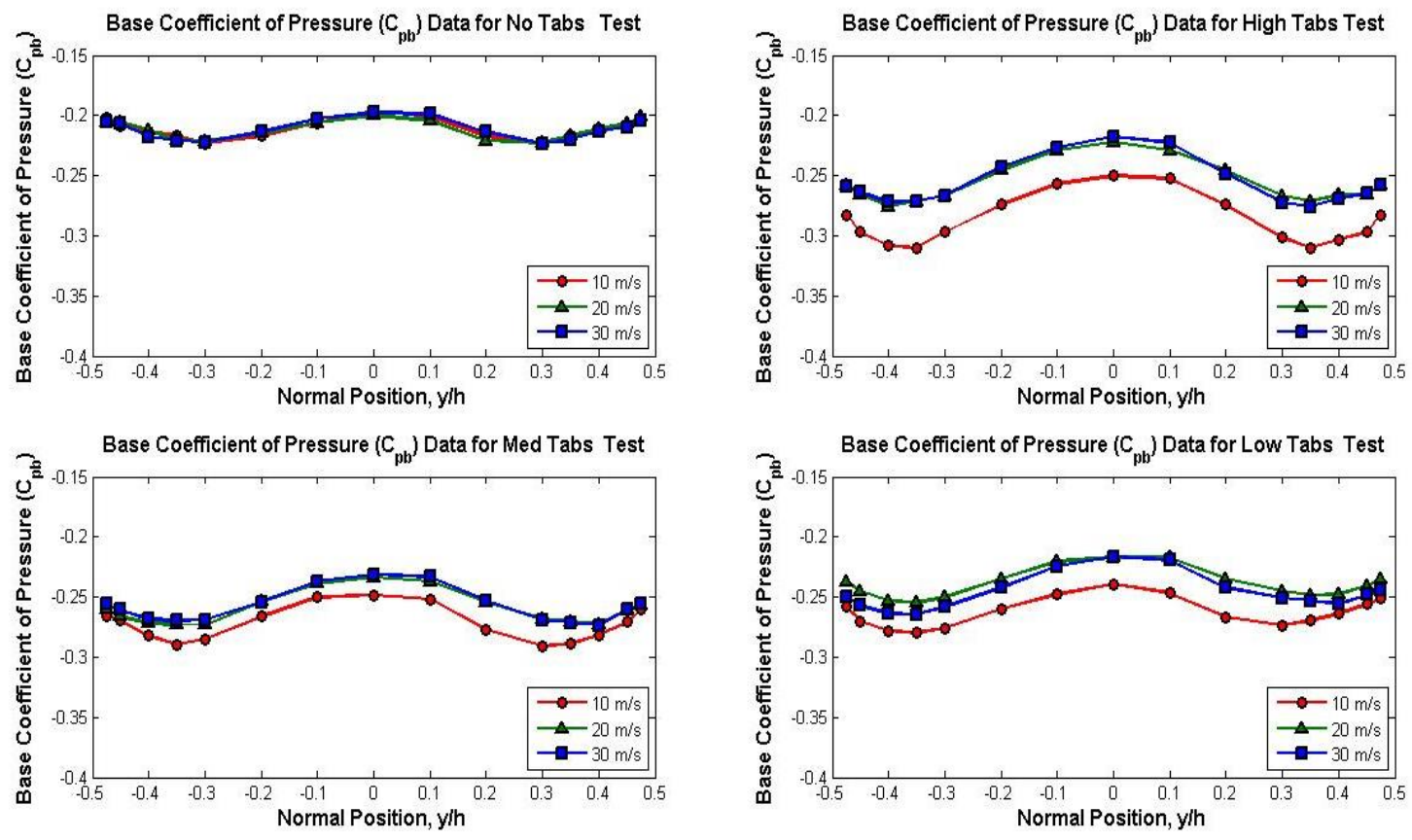

Fig. 31. Normal base $\mathrm{C}_{\mathrm{Pb}}$ distribution for each tab height with varying speeds.

Fig. 31 shows the same normal position data organized by tab height with varying speeds. In these figures it is shown that for all four tab configurations there is a trend with speed. This trend is that the $10 \mathrm{~m} / \mathrm{s}$ tests have the lowest average $C_{\mathrm{Pb}}$ while the $20 \mathrm{~m} / \mathrm{s}$ and $30 \mathrm{~m} / \mathrm{s}$ tests have very similar average $\mathrm{C}_{\mathrm{Pb}}$. 


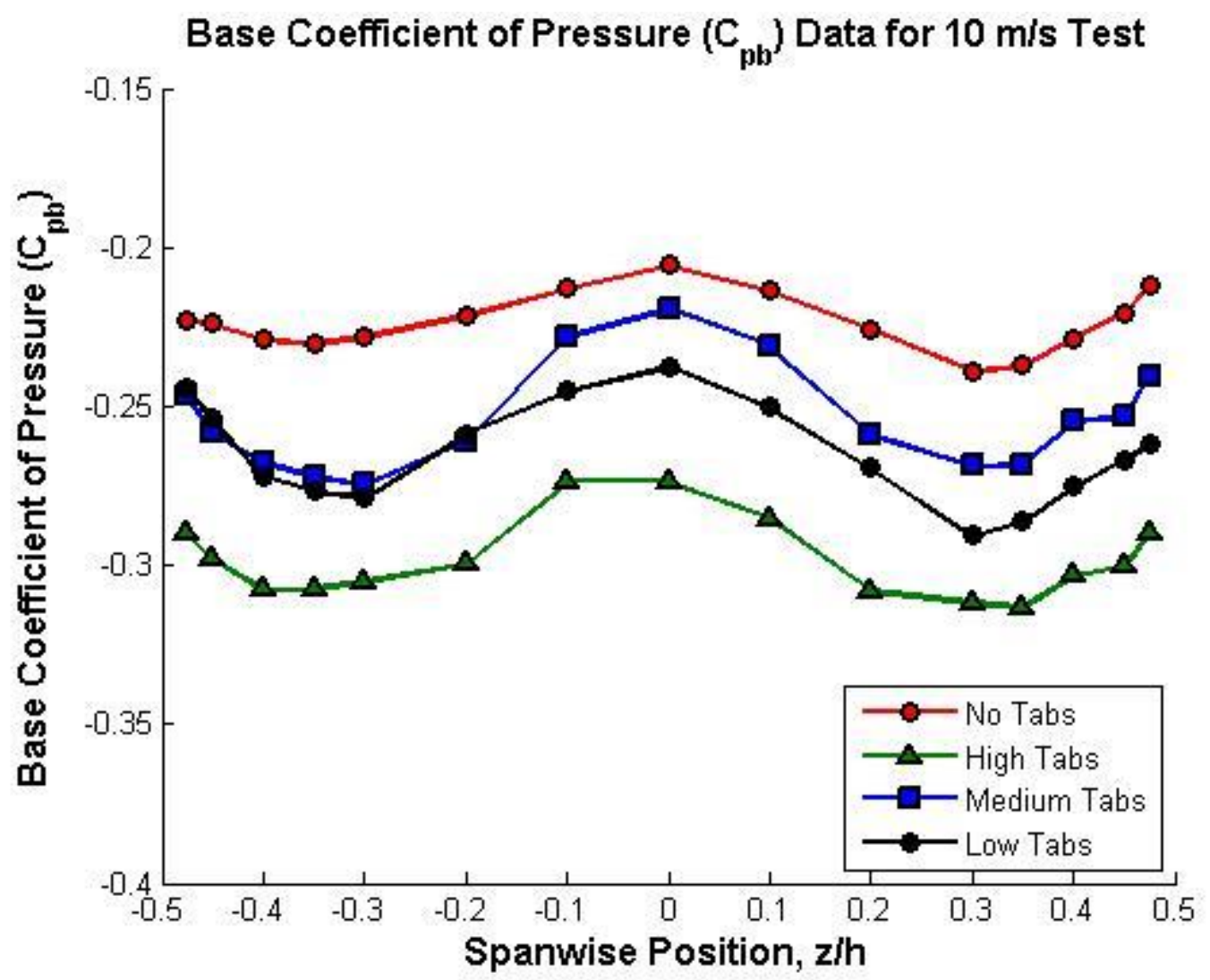

Fig. 32. Spanwise base pressure results for $10 \mathrm{~m} / \mathrm{s}$ tests.

Fig. 32 shows the base pressure resulted in the spanwise direction on the centerline of the model for the $10 \mathrm{~m} / \mathrm{s}$ case. The results above show similar results to the $10 \mathrm{~m} / \mathrm{s}$ normal direction tests. In this test it is shown that the non-tabbed case has the highest average $\mathrm{C}_{\mathrm{Pb}}$ and has the shallowest valleys. These two results show that the tabs increase not only the vortex strength in the normal direction but also in spanwise direction. They suggest an increase in the mean drag on the model for this speed. 


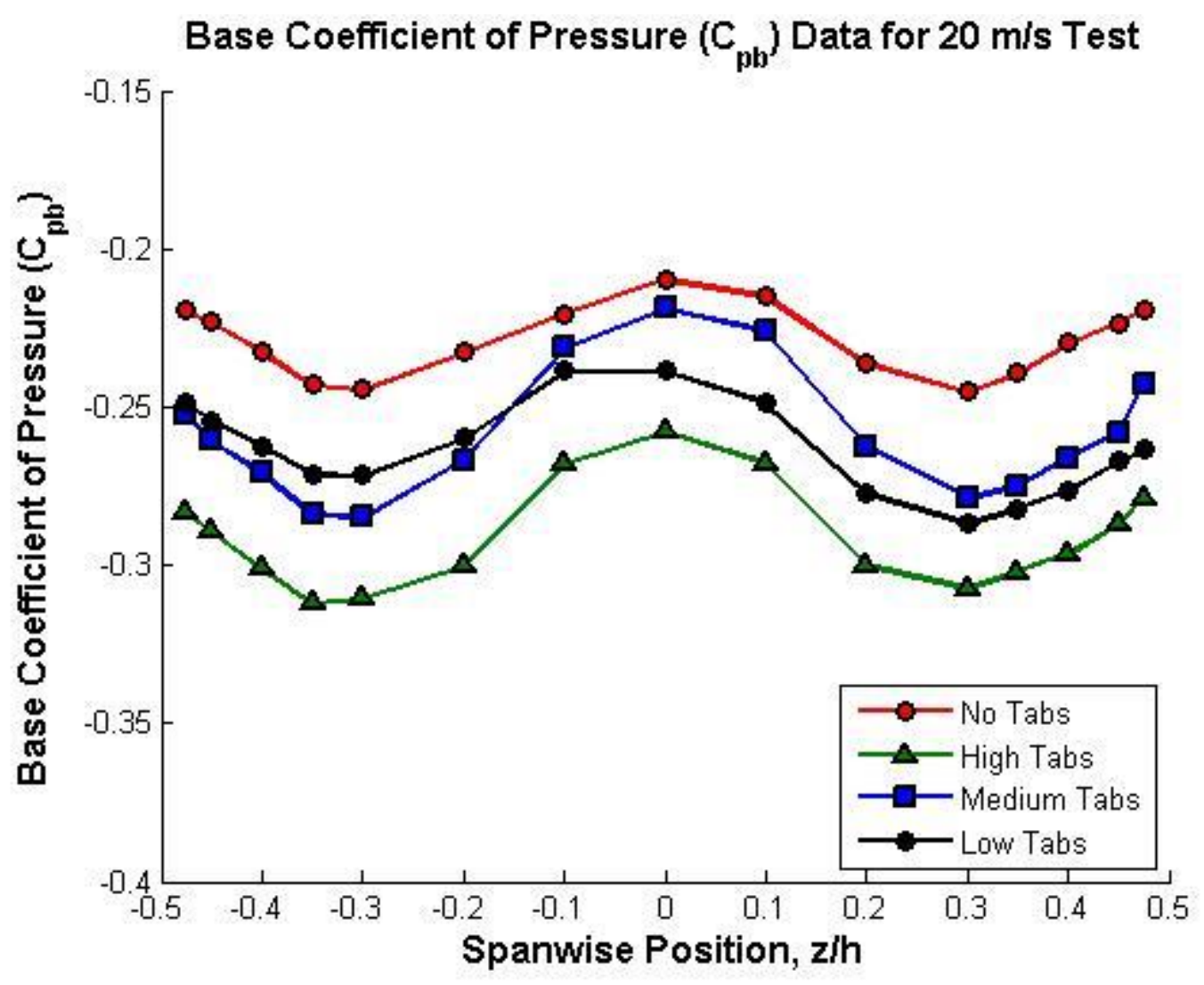

Fig. 33. Spanwise base pressure results for $20 \mathrm{~m} / \mathrm{s}$ tests.

Fig. 33 shows the base pressure results in the spanwise direction on the centerline of the model for the $20 \mathrm{~m} / \mathrm{s}$ case. The results above are similar to the $20 \mathrm{~m} / \mathrm{s}$ normal direction tests. In this test it is shown that the non-tabbed case has the highest average $\mathrm{C}_{\mathrm{Pb}}$ and has the shallowest valleys. These two results prove that the tabs increase the vortex strength and suggest an increase in the mean drag on the model for this speed. 


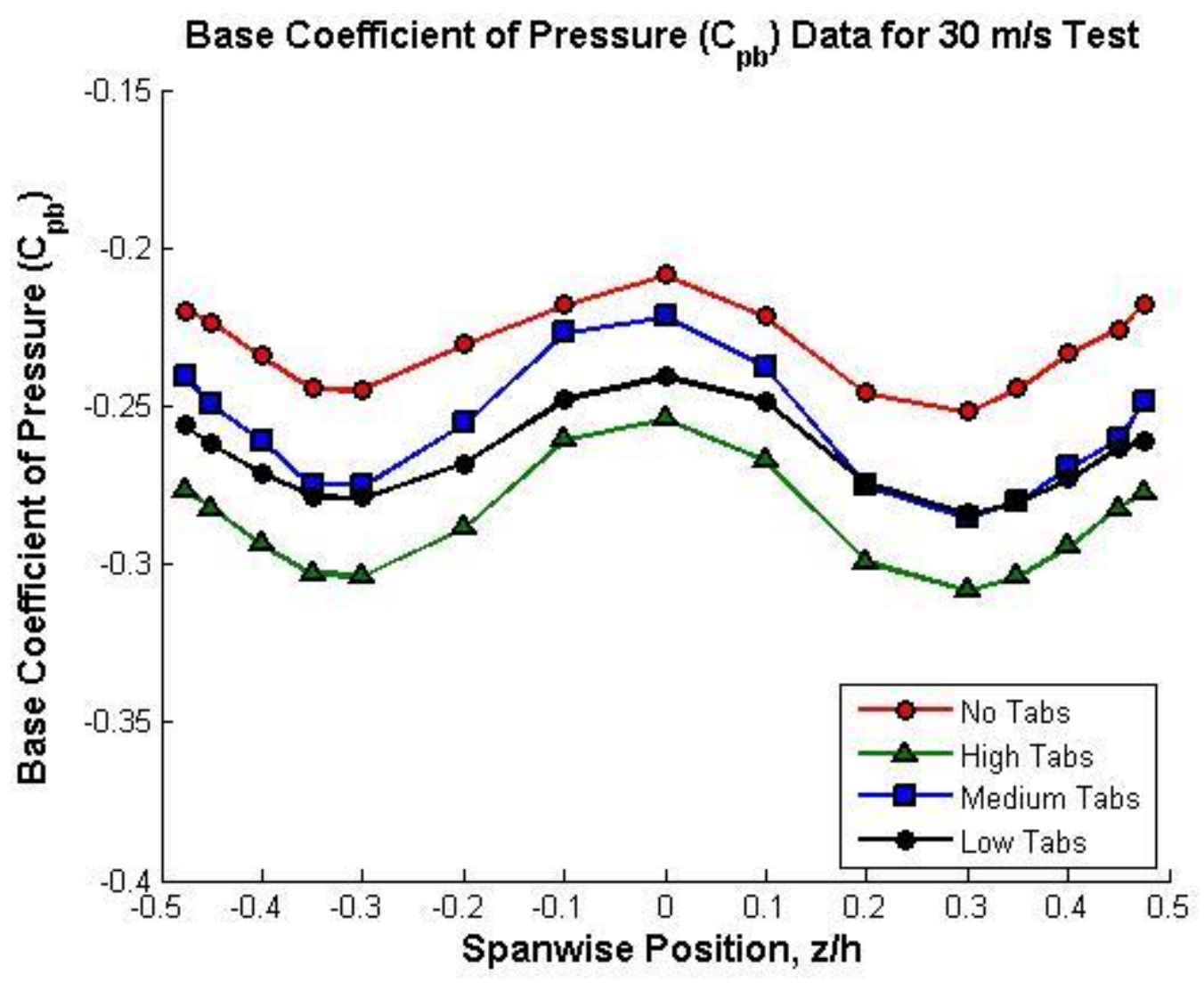

Fig. 34. Spanwise base pressure results for $30 \mathrm{~m} / \mathrm{s}$ tests.

Fig. 34 shows the base pressure results in the spanwise direction on the centerline of the model for the $30 \mathrm{~m} / \mathrm{s}$ case. The results above are similar to the $30 \mathrm{~m} / \mathrm{s}$ normal direction tests. In this test it is shown that the non-tabbed case has the highest average $\mathrm{C}_{\mathrm{Pb}}$ and has the shallowest valleys. 

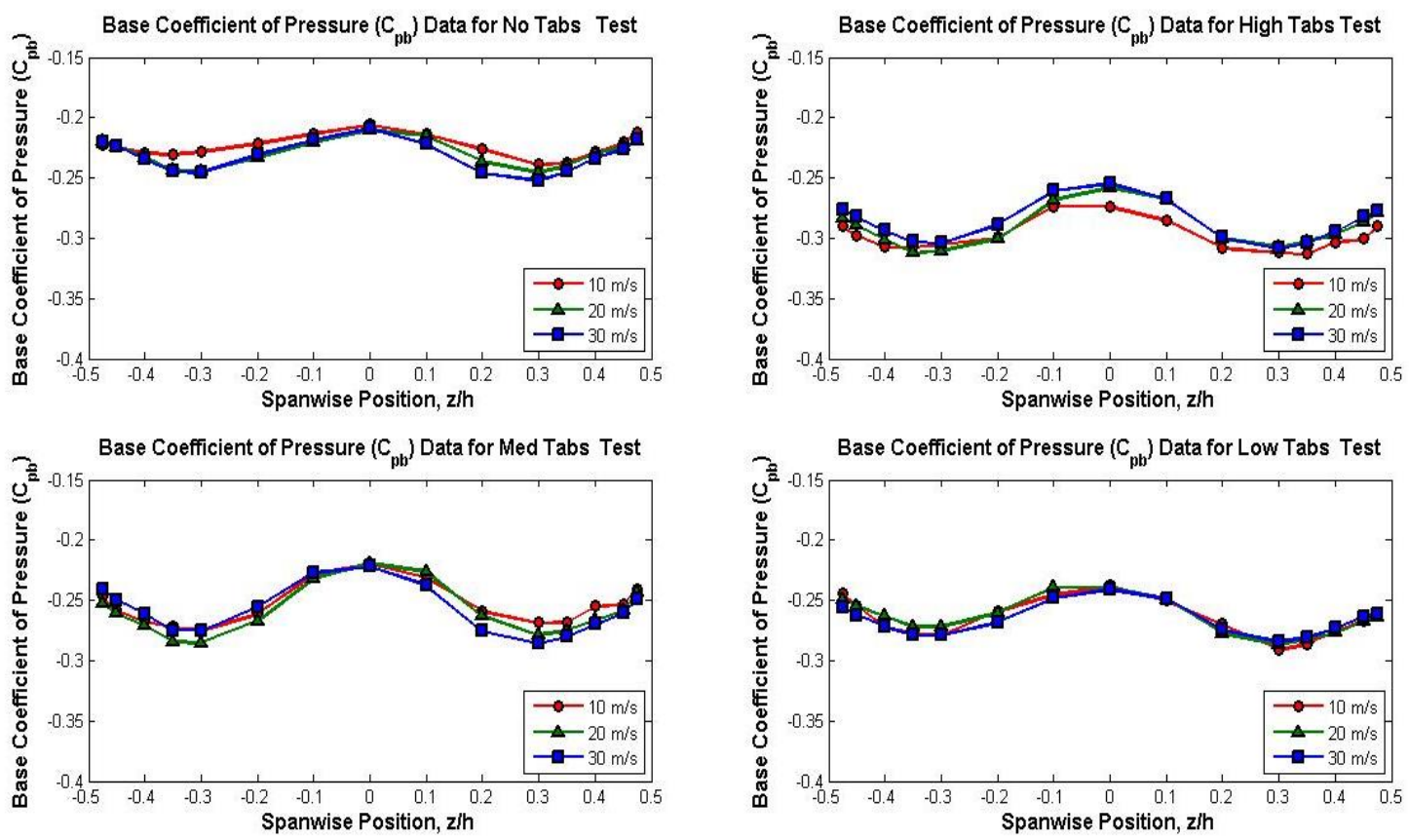

Fig. 35. Spanwise base $C_{p b}$ distribution for each tab height with varying speeds.

In the experiment by Park et al. [3] and in Pinn's experiment [6] the spanwise $\mathrm{C}_{\mathrm{Pb}}$ data was almost perfectly flat. In Park's et al.'s data this was due to the model spanning the entire tunnel and thus there was no flow in the spanwise direction. In Pinn's experiment this was due to the high aspect ratio. In this experiment, even the non-tabbed tests show the two valleys which indicate vortex shedding. This result is very significant because it shows that with a low aspect ratio cross section there is a spanwise vortex that needs to be accounted for.

The spanwise data in Fig. 35 shows similar trends to the normal data. For all three test speeds, Fig. 32-Fig. 34 show that the non-tabbed case has the highest average $\mathrm{C}_{\mathrm{Pb}}$. This is consistent with the normal row tests and suggests an increase in suction on the model and an increase in mean drag with the addition of the tabs. The spanwise data also shows a distinct increase in the depth of the valleys. The spanwise data shows a greater increase in 
valley depth than in the normal data. This increase in valley depth shows the existence of a spanwise vortex with and without the tabs.

\subsection{Sting Balance Drag Results}

Before the total drag force tests could be started, the sting balance had to be calibrated. This was done using the methods described in the procedures section. By adding weight to the sting balance and taking a data point for each incremental weight, the calibration curve in Fig. 36 was calculated.

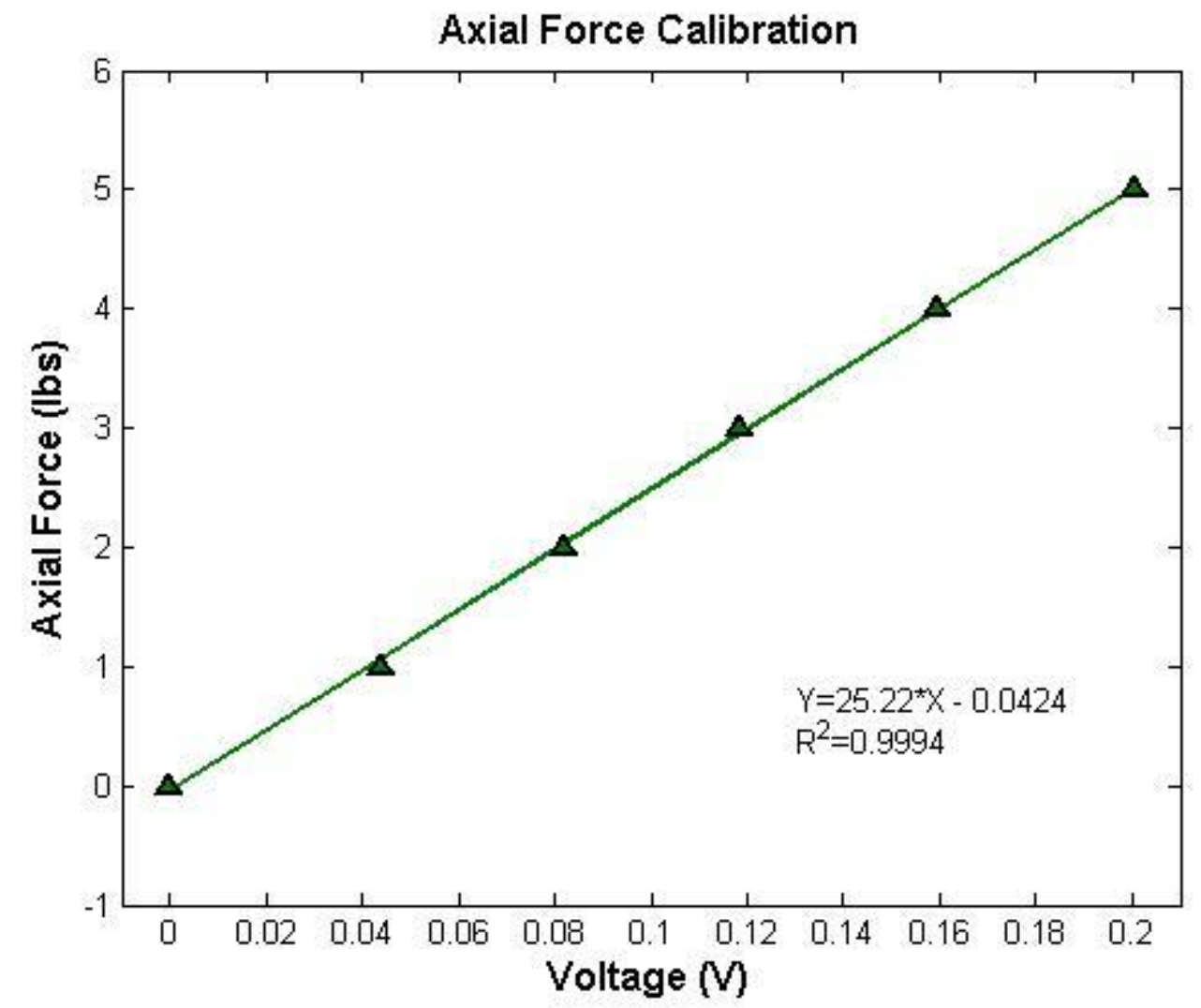

Fig. 36. Sting balance calibration curve.

The equation in Fig. $\mathbf{3 6}$ shows the relation between voltage $(X)$ and the axial force on the sting balance ( $Y$ ). The $\mathrm{R}^{2}$ value in Fig. $\mathbf{3 6}$ shows that the curve is almost perfectly linear. This linearity shows that the sting balance was calibrated and functioning correctly. Once the calibration was completed, the calibration weights and pulley were 
removed from the tunnel and the model was secured to the sting mount. To take a baseline measurement, the forces on the sting were measured without any flow in the tunnel. These measurements show the forces caused by the weight of the model. This was done for each configuration. Next, force data was taken for all four tab configurations at the three test speeds. The baseline measurement was then subtracted from the forces caused by the flow to calculate the force on the model caused only by the flow.

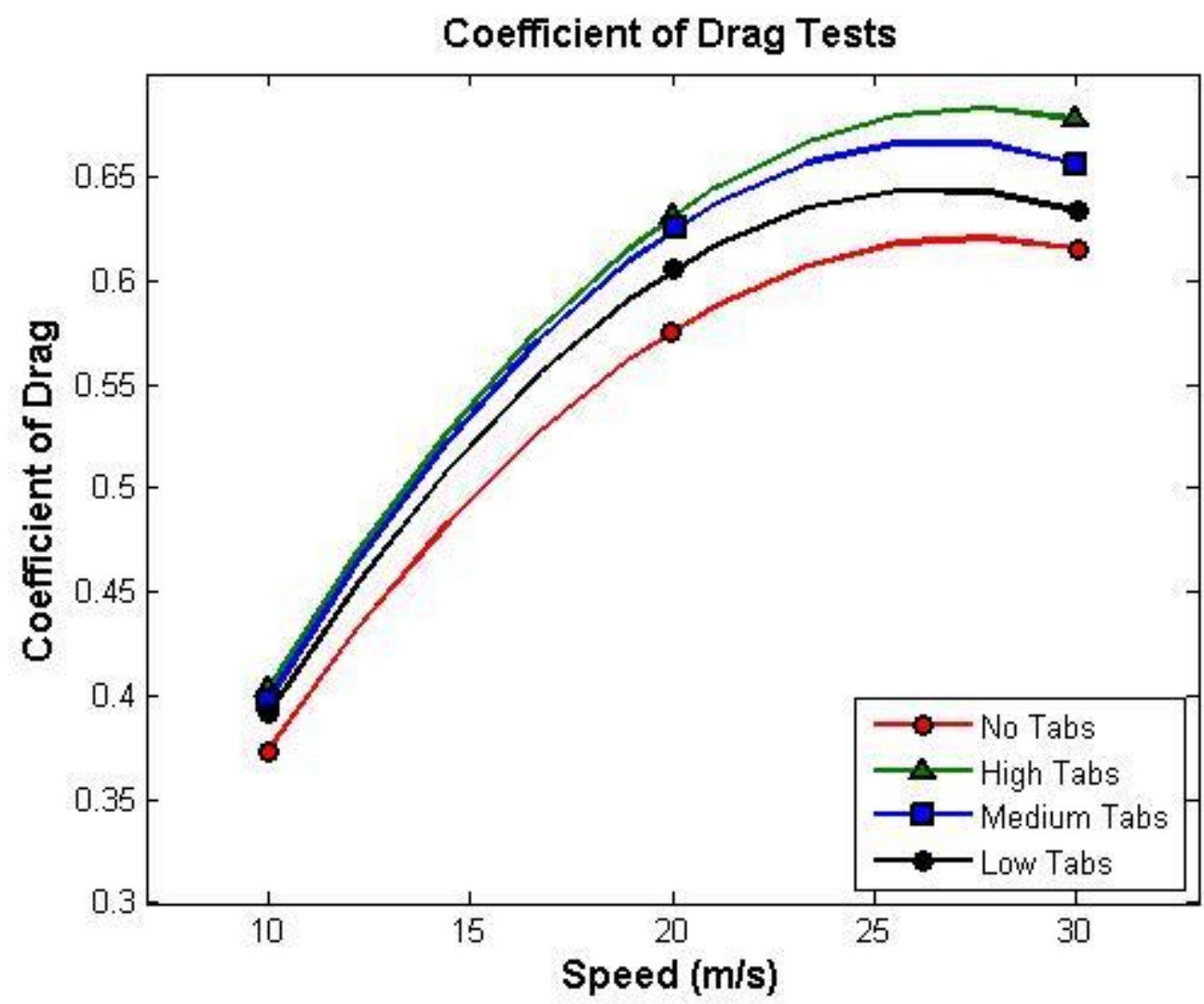

Fig. 37. Coefficient of drag results for all four tab configurations at all three speeds.

The coefficient of drag was then calculated using the force caused only by the flow. Because the model was set to zero degrees angle of attack and zero degrees yaw with respect to the flow, only the axial force was required to get the coefficient of drag using Equ. (10).

$$
C_{D}=\frac{D}{q_{\infty} * S}
$$


where $D$ is the axial force measured by the sting balance, $q_{\infty}$ is the free stream dynamic pressure measured by the Pitot-static probe, and $S$ is the cross sectional (reference) area of the model.

The data shown in Fig. 37 agrees with the base pressure results by showing that the drag on the body increases as the tabs are added. It follows the previous experiment's trend in that increasing the speed also increases the drag coefficient on the model [6]. A spline curve is fit to the data to keep it consistent with previous experimental results. The drag curve is consistent with previous experiments because the increase in drag from 20 $\mathrm{m} / \mathrm{s}$ to $30 \mathrm{~m} / \mathrm{s}$ is much smaller than the increase from $10 \mathrm{~m} / \mathrm{s}$ to $20 \mathrm{~m} / \mathrm{s}$ [6]. The current data differs from the previous experiments [6] by showing that the tabs increase the total drag instead of decrease total drag. As with the $\mathrm{C}_{\mathrm{Pb}}$ data in Table 2, Table 3 shows the same trend in that decreasing tab height creates a reduction in drag.

Table 3. Change in $C_{D}$ with Tabs Compared to No Tab Configuration.

\begin{tabular}{|c|c|c|c|}
\hline & $10 \mathrm{~m} / \mathrm{s}$ & $20 \mathrm{~m} / \mathrm{s}$ & $30 \mathrm{~m} / \mathrm{s}$ \\
\hline Low Tabs & $+4.9 \%$ & $+5.1 \%$ & $+2.9 \%$ \\
\hline Medium Tabs & $+6.6 \%$ & $+8.7 \%$ & $+6.6 \%$ \\
\hline High Tabs & $+8.1 \%$ & $+9.8 \%$ & $+10.2 \%$ \\
\hline
\end{tabular}

\subsection{Hotwire Energy Spectra}

Before any hotwire tests could be conducted, the hotwire probe needed to be calibrated. This was done by placing the hotwire in the calibrator and releasing a jet of air with a known speed over the probe and measuring the voltage. Using ThermalPro to measure the differential pressure of the flow over the probe and the voltage required to maintain the temperature of the wire, the curve below was created. 


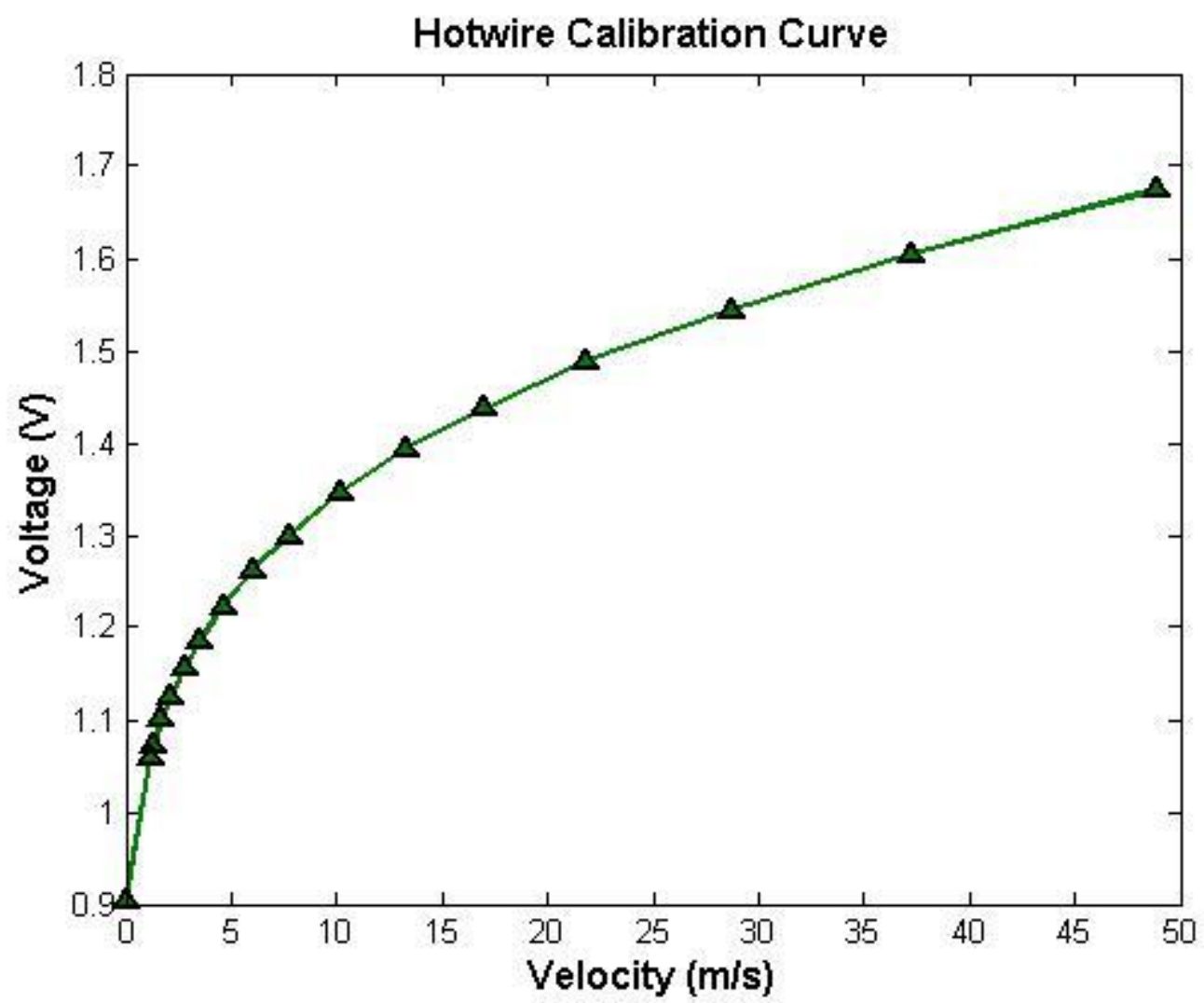

Fig. 38. Calibration curve for the hotwire probe.

The calibration curve above in Fig. 38 yields this fourth order correlation equation.

$$
v=-6.4+146.3 * V-384.5 * V^{2}+363.4 * V^{3}-118.2 * V^{4}
$$

Where $v$ is the speed across the probe and $V$ is the measured voltage. Though the independent variable for the actual testing is the measured voltage, the calibration curve was setup this way because during calibration the speed was a known value.

Once the hotwire was calibrated it was removed from the calibrator and placed into the wind tunnel. The hotwire was placed even with the edge of the model in the normal direction, directly on the centerline in the spanwise direction, and $0.0254 \mathrm{~m}$ ( 1 inch) behind the center tab in the streamwise direction. This corresponds to $\mathrm{x} / \mathrm{h}=0.5, \mathrm{y} / \mathrm{h}=0$, and $\mathrm{z} / \mathrm{h}=0.2$. Once the probe was in place, ThermalPro was used to measure the spectral density of the flow at all of the testing configurations and speeds. This spectral density 
measures the fluctuations in the flow by comparing the frequency to the magnitude of the measured voltages. The spectral density was then converted into the Strouhal Number using Equ. (12) and the energy spectrum using Equ. (13).

$$
S t=\frac{f * h}{U_{\infty}}
$$

where $f$ is the frequency measured in ThermalPro, $h$ is the height of the model $0.127 \mathrm{~m}(5$ inches), and $U_{\infty}$ is the free stream velocity.

$$
E S=\frac{E(f)}{U_{\infty}^{2}}
$$

where $E S$ is the normalized energy spectrum, $E(f)$ the spectral energy measured in ThermalPro and $U_{\infty}$ the free stream velocity.

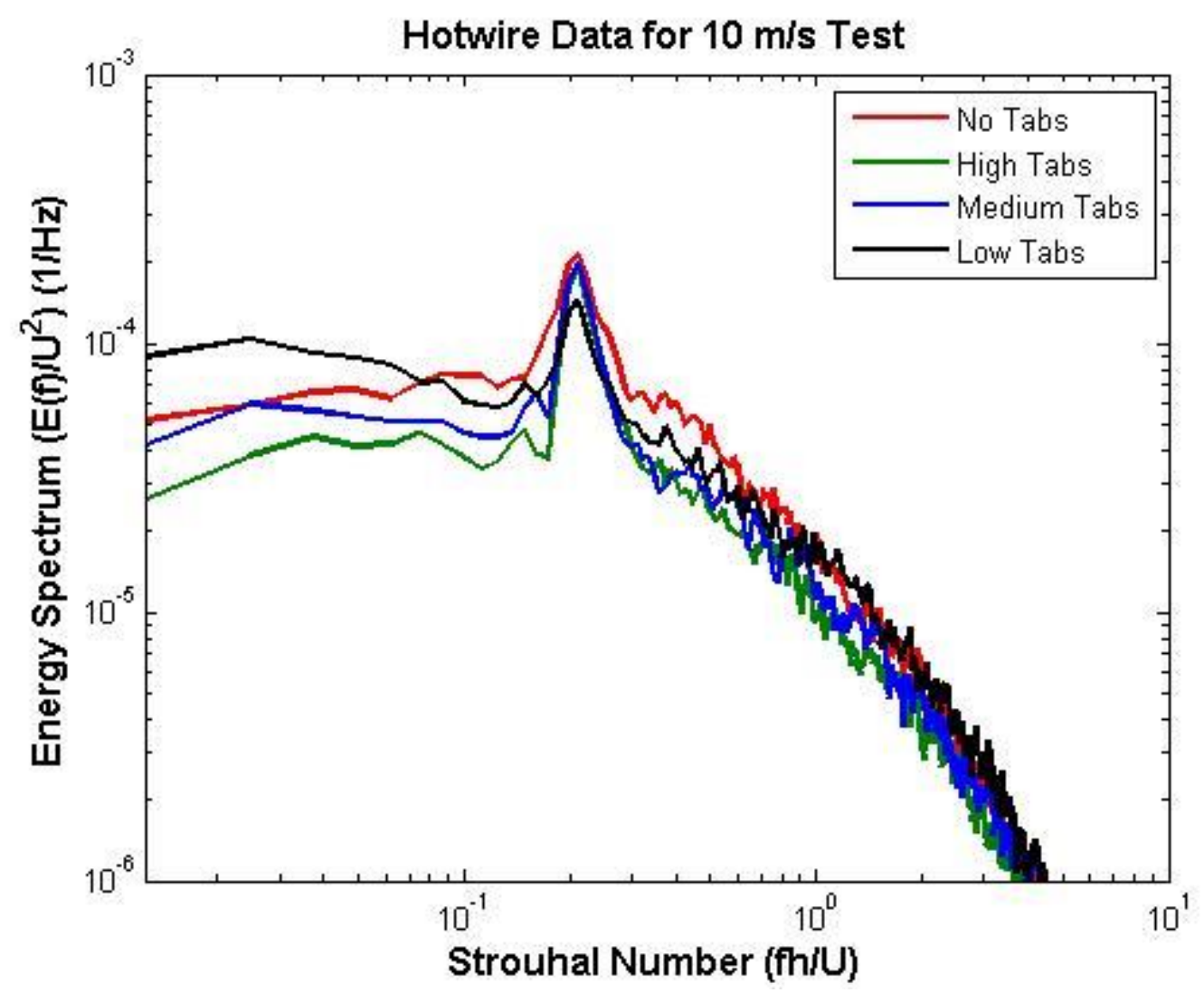

Fig. 39. Energy spectra results for $10 \mathrm{~m} / \mathrm{s}$ speed for all four tab configurations. 
Fig. 39 shows the energy spectrum of the wake flow behind the model for the $10 \mathrm{~m} / \mathrm{s}$ test cases. For all four tab configurations there is a large spike close to the Strouhal number of 0.21 . The large width of the spike is due to the low resolution of the energy spectrum at lower Strouhal Numbers. This large spike shows that there is a vortex forming in the wake of the model. This data matches the base pressure data because it shows that a vortex was shedding for all of the test cases. This result validates the results from the base pressure tests because it shows that the vortex is not attenuated with the tabs.

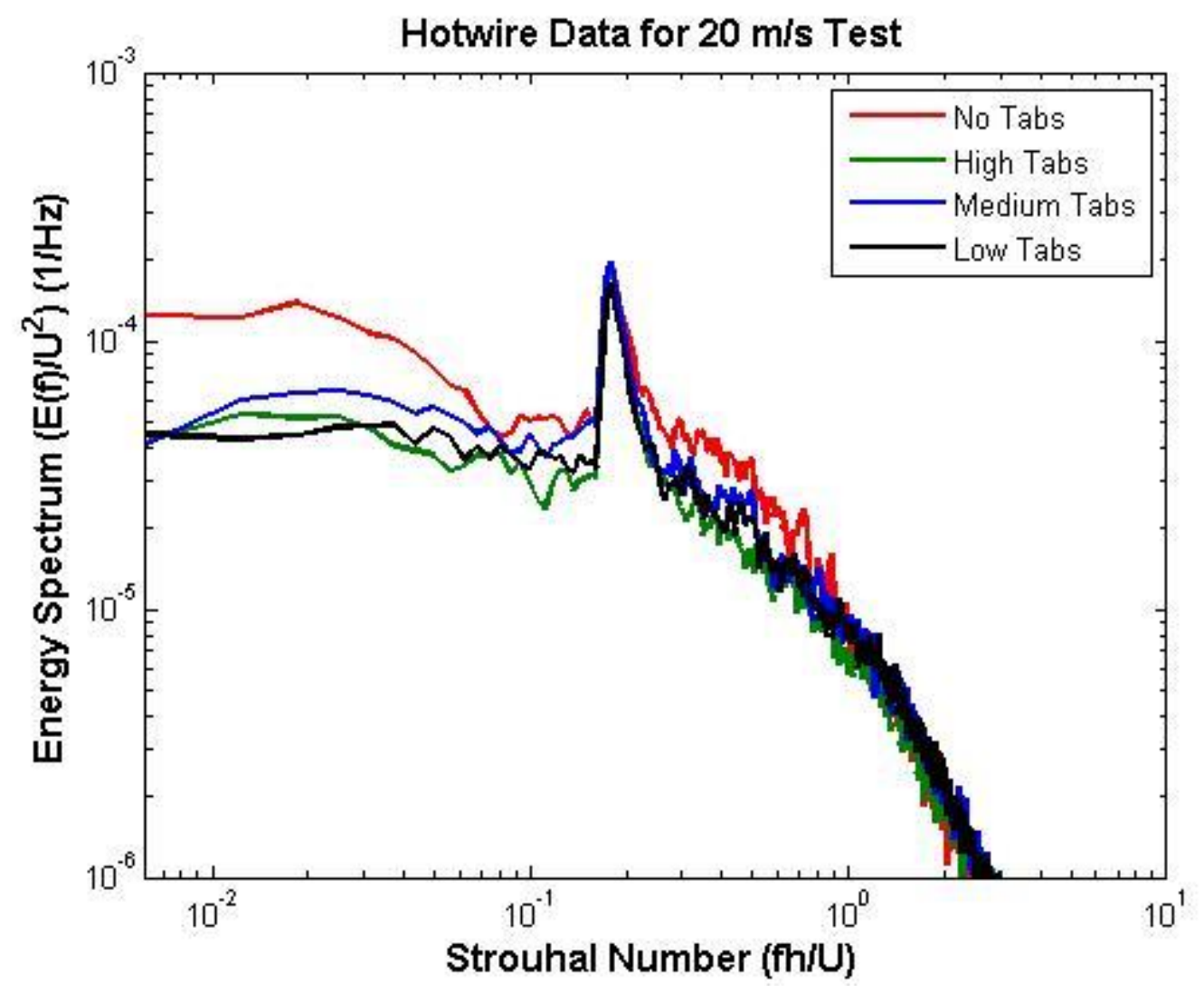

Fig. 40. Energy spectra results for $20 \mathrm{~m} / \mathrm{s}$ speed for all four tab configurations.

Fig. 40 shows the energy spectrum of the wake flow behind the model for the $20 \mathrm{~m} / \mathrm{s}$ test cases. For all four tab configurations there is a large spike close to the Strouhal number of 0.18 . As with previous experiments the spike moves slightly to the left and 
becomes narrower with the increase in speed from $10 \mathrm{~m} / \mathrm{s}$ to $20 \mathrm{~m} / \mathrm{s}$. The spike is narrower than the $10 \mathrm{~m} / \mathrm{s}$ tests because the data resolution increases with an increase in Strouhal Number. This large spike shows that there is a vortex forming in the wake of the model. This data matches the base pressure data because it shows that a vortex is shedding for all of the test cases. This result validates the results from the base pressure tests because it shows that the vortex is not attenuated with the tabs.

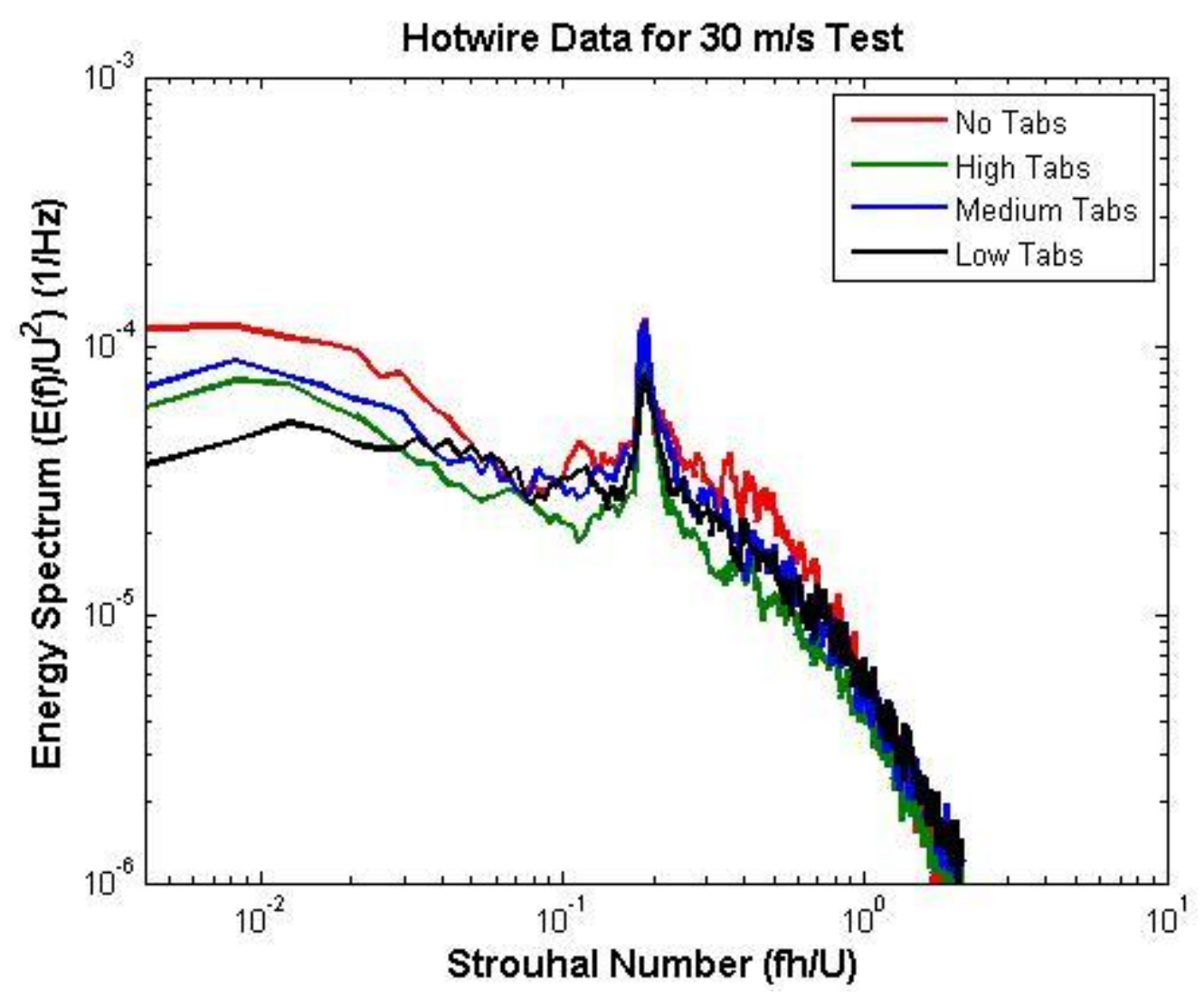

Fig. 41. Energy spectra results for $30 \mathrm{~m} / \mathrm{s}$ speed for all four tab configurations.

Fig. 41 shows the energy spectrum of the wake flow behind the model for the $30 \mathrm{~m} / \mathrm{s}$ test cases. For all four tab configurations there is a large spike close to the Strouhal number of 0.19. As with previous experiments the spike moves slightly to the right and becomes thicker with the in increased speed from $20 \mathrm{~m} / \mathrm{s}$ to $30 \mathrm{~m} / \mathrm{s}$. This large spike shows that there is a vortex forming in the wake of the model. This data is aligned with 
the base pressure data because it shows that a vortex is shedding for all of the test cases. This result validates the results from the base pressure tests because it shows that the vortex is not attenuated with the tabs. Though the hotwire tests were not necessary because of the results from the base pressure tests, the hotwire tests act as a second source to prove that the tabs did not attenuate the vortex for this experiment. 


\section{DISCUSSION}

The results with the addition of the low, medium, and high tabs for the square base pressure tests, the sting balance tests, and the hotwire energy spectra tests all suggest that the tabs increase vortex strength instead of reducing it. This is apparent by the decrease in average base pressure, the increase in total drag and the existence of a large spike in the hotwire energy spectrum.

In the experiment by Park et al. [3], it was shown that the vortex was attenuated by the increase of the wake width behind the test model. This increase in wake width was caused by the generation of streamwise vortices created by the tabs. The addition of the tabs in previous experiments $[3,6]$ resulted in an increase in base pressure. In the 2D bluff base case, the tabs prolong the formation of the vortex, decrease the suction on the model and thus increase the base pressure [3]. In the rectangular bluff base with an aspect ratio of four, the base pressure was reported to be increased by adding tabs only on the long side of the base [6]. This base pressure recovery was not observed in this experiment.

In Pinn's experiment the vortices in the normal direction (normal vortices) were four times longer than the vortices in the spanwise direction (spanwise vortices) and the normal vortices were much weaker so controlling vortices only in the normal direction was sufficient to prolong the vortex formation [6] for the lower test speeds. For the present experiment, however, the spanwise and normal vortices are equal in length and almost equal in strength due to the similar boundary layer vorticity development. The equality in length is shown in Fig. $\mathbf{4 2}$ and the similarity in strength can be seen in the base pressure testing in Fig. 28 -Fig. 35. 


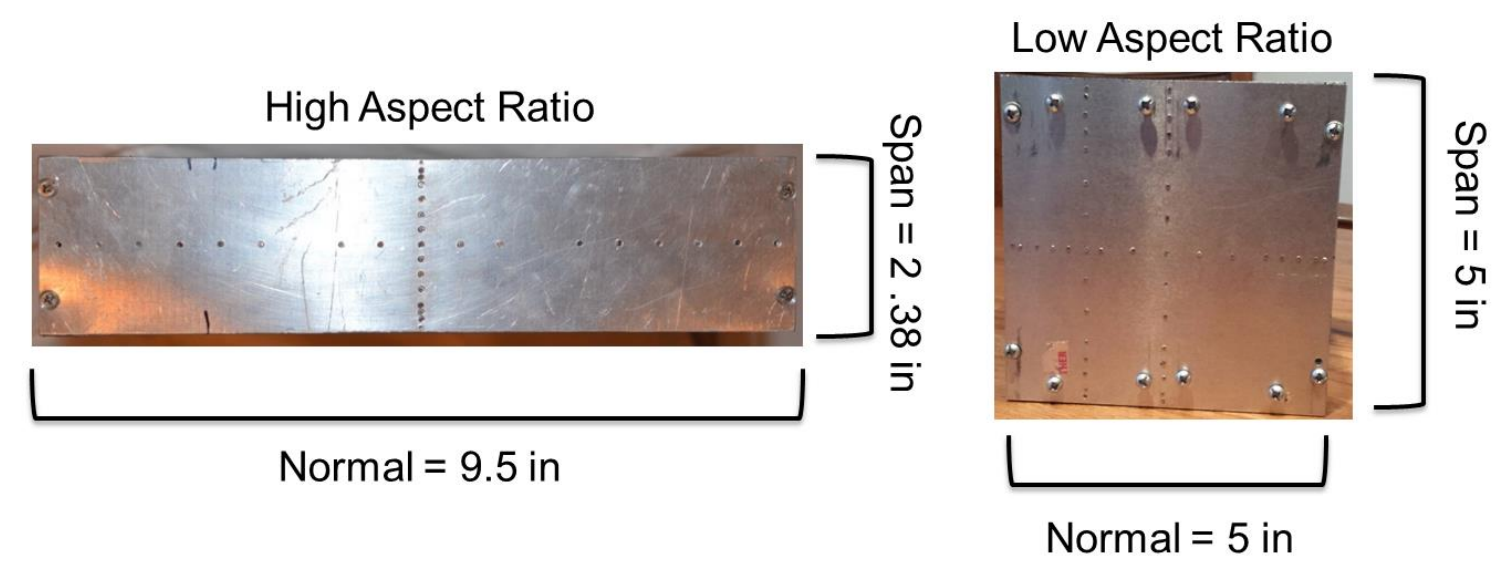

Fig. 42. Comparison of model aspect ratios.

In this case the conjecture is that when the tabs are introduced in only the normal direction, the edges of the two side tabs (the tabs close to the edges of the square base) produce vortices which are close to and in the same directions as the spanwise vortices, illustrated in Fig. 43 below. As a result, the strength of the spanwise vortices increase, as do the strength of the normal vortices. The resulting decrease in base pressure causes the increase in drag. Therefore, for the square base model, further study needs to be done with the tabs introduced in both the normal and spanwise directions to show its drag reduction effect and possibly in different tab locations.

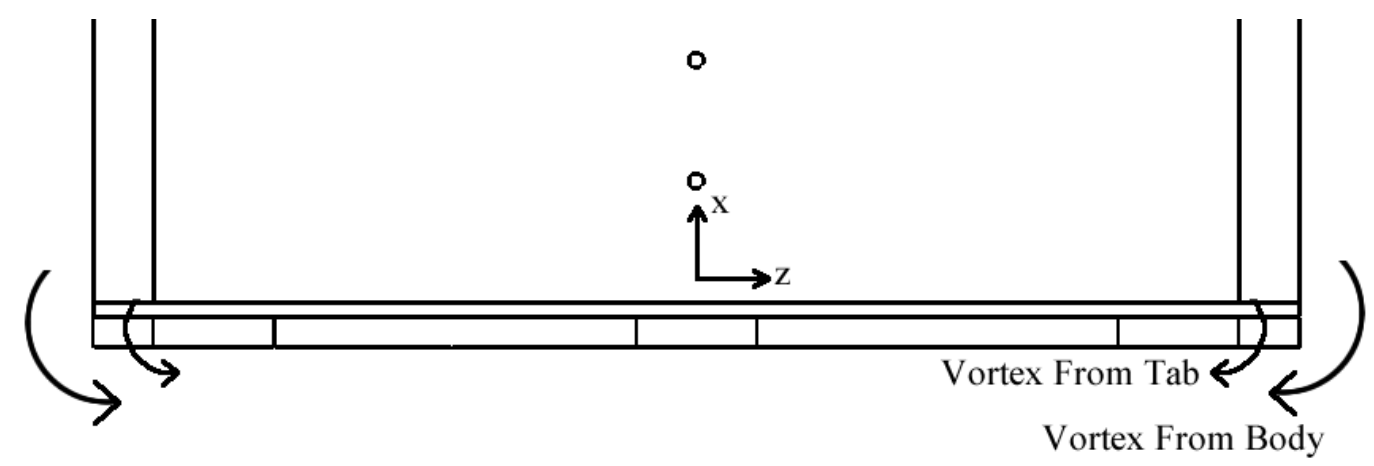

Fig. 43. Schematic of vortices. 


\section{CONCLUSIONS}

A square base bluff body was tested in the Cal Poly $3 \times 4 \mathrm{ft}$ low-speed wind tunnel. The tests aimed to show the effectiveness of adaptive end plate tabs to reduce drag on the bluff body model. The goal of the tabs was to increase the base pressure and decrease the mean drag while attenuating the vortex shedding in the wake of the model. Unlike Pinn's experiment on a bluff body with the aspect ratio equal to 4 , by merely disturbing the vortex with tabs in the spanwise direction, no drag reduction was found. For the present model with aspect ratio equal to 1 though, the tabs increased the drag on the model at all three tested Reynolds numbers. This analysis was done by first measuring the boundary layer on the trailing edge of the model to find the optimal tab height. The base pressure was then tested to see the effects of the tabs on the base pressure. Next a total force measurement was taken at all speeds and tab configurations to calculate the total drag on the model. Finally a hotwire probe was used to measure the velocity fluctuations in the wake flow near the model. These tests were done at speeds of 10,20 , and $30 \mathrm{~m} / \mathrm{s}$ which correlate to Reynolds numbers of $8.3 \mathrm{e} 4,1.6 \mathrm{e} 5$ and $2.5 \mathrm{e} 5$. The three conclusions are as follows:

1. Base pressure measurements showed a decrease in base pressure at an average of $0.0449,0.0398$, and 0.0394 for 10,20 , and $30 \mathrm{~m} / \mathrm{s}$ respectively by adding the tabs to the trailing edge. This correlates to an increase in mean drag. Base pressure testing also showed an increase in vortex strength instead of a decrease due to larger valleys in the $\mathrm{C}_{\mathrm{pb}}$ data.

2. Direct total drag force measurements also showed an increase in overall drag of the model with the addition of the tabs for all tab heights and test speeds. 
3. Hotwire data showed that the vortex is present with and without the tabs. This result was shown by a large spike in the energy spectrum near a Strouhal number of 0.2 .

In previous works it was shown that the mechanism for drag reduction by tabs was to increase the wake width between the tabbed edges [3]. While it appears the flow was altered with the addition of the tabs on the present model, the drag was still increased. This was possibly due to the strengthening of spanwise vortices. For future testing in this field, tabs should be added to all four edges of the low aspect ratio bluff body at different locations in order to see the tab effects in attenuating the vortices. 


\section{REFERENCES}

1 Bearman, P. W. Investigation of the flow behind a two-dimensional model with a blunt trailing edge and fitted with splitter plates. J. Fluid Mech. (1995), 241-255.

2 Bearman, P. W. The Effect of Base Bleed on the Flow behind a Two-Dimensional Model with a Blunt Trailing Edge. The Aeronautical Quarterly Vol. XVIII (1967), 207-224.

3 Park, H, Lee, D, Jeon, W-P, Hahn, S, and Kim, J. Drag reduction in flow over a twodimensional bluff body with a blunt treailing edge using a new passive device. The Journal of FLuid Mechanics (2006), 389-414.

4 Knight, James and Tso, Jin. A Comparison of Bluff-Body Base-Drag Reduction By Passive Control Means. Ontario, 2005.

5 Knight, James. Drag Reduction for Reusable Launch Vehicles Through Boundary Layer Extraction. 2003.

6 Pinn, Jarred. Effect of End-Plate Tabs on Drag Reduction of a 3D Bluff Body with a Blunt Base. San Luis Obispo, 2012.

7 Carlson, Charles and Innes, Paul. Test and Verification of Vortex Shedding for a 3D Bluff Body. San Luis Obispo, 2012.

8 Erlhoff, Ethan. Distributed Forcing on A 3D Bluff Body With A Blunt Base An Experimental Active Drag Control Approach. San Luis Obispo, 2012.

9 IFA 300 Constant Temperature ANemometer System Instruction Manual. TSI Incorporated, St. Paul, 2000.

10 Pope, Alan. Wind-Tunnel Testing. John Wiley \& Sons, New York, 1954.

11 Taylor, John R. An Introduction to Error Analysis: The Study of Uncertainties in Physical Measurements. University Science Books, 1997. 


\section{APPENDICES}

\section{A. Sample Error Calculations}

The following calculations were derived from methods by Taylor [11].

$$
\begin{aligned}
& C_{P}=\frac{P-P_{\infty}}{q_{\infty}}=\frac{P-P_{\infty}}{P_{t}-P_{\infty}} \\
& \frac{d C_{P}}{C_{P}}=\frac{d\left(P-P_{\infty}\right)}{P-P_{\infty}}-\frac{d\left(P_{t}-P_{\infty}\right)}{P_{t}-P_{\infty}}=\frac{d P}{P-P_{\infty}}-\frac{d P_{\infty}}{P-P_{\infty}}-\frac{d P_{t}}{P_{t}-P_{\infty}}+\frac{d P_{\infty}}{P_{t}-P_{\infty}} \\
& \frac{d C_{P}}{C_{P}} \approx \frac{d P}{P-P_{\infty}}+\left(\frac{-1}{P-P_{\infty}}+\frac{1}{P_{t}-P_{\infty}}\right) d P_{\infty}-\frac{d P_{t}}{P_{t}-P_{\infty}} \\
& \sigma_{C_{P}} \approx\left|C_{P}\right| \sqrt{\left(\frac{\sigma_{P}}{P-P_{\infty}}\right)^{2}+\left[\left(\frac{-1}{P-P_{\infty}}+\frac{1}{P_{t}-P_{\infty}}\right) \sigma_{P_{\infty}}\right]^{2}+\left(\frac{\sigma_{P_{t}}}{P_{t}-P_{\infty}}\right)^{2}} \\
& C_{P}=\frac{P-P_{\infty}}{P_{t}-P_{\infty}}=\frac{-0.1113-0.0930}{-0.0063-0.0930}=-0.2111 \\
& \left(\frac{4.0415 * 10^{-5}}{-0.1113-0.0930}\right)^{2}+\cdots \\
& \sigma_{C_{P}} \approx|-0.2111| \mid\left[\left(\frac{-1}{-0.1113-0.0930}+\frac{1}{-0.0063-0.0930}\right) 3.2112 * 10^{-4}\right]^{2}+\cdots \\
& \ldots\left(\frac{2.8868 * 10^{-5}}{-0.0063-0.0930}\right)^{2}
\end{aligned}
$$

$\sigma_{C_{P}} \approx 0.0211$

Similarly,

$$
\begin{aligned}
& C_{D}=\frac{D}{q_{\infty} A}=\frac{D}{\left(P_{t}-P_{\infty}\right) A} \\
& \frac{d C_{D}}{C_{D}}=\frac{d D}{D}-\frac{d\left(P_{t}-P_{\infty}\right)}{P_{t}-P_{\infty}}-\frac{d A}{A}=\frac{d D}{D}-\frac{d P_{t}}{P_{t}-P_{\infty}}+\frac{d P_{\infty}}{P_{t}-P_{\infty}}-\frac{d A}{A}
\end{aligned}
$$

Neglect A, so

$\frac{d C_{D}}{C_{D}} \approx \frac{d D}{D}-\frac{d P_{t}}{P_{t}-P_{\infty}}+\frac{d P_{\infty}}{P_{t}-P_{\infty}}$

So

$$
\begin{aligned}
& \sigma_{C_{D}} \approx\left|C_{D}\right| \sqrt{\left(\frac{\sigma_{D}}{D}\right)^{2}+\left(\frac{\sigma_{P_{t}}}{P_{t}-P_{\infty}}\right)^{2}+\left(\frac{\sigma_{P_{\infty}}}{P_{t}-P_{\infty}}\right)^{2}} \\
& C_{D}=\frac{D}{q_{\infty} * S}=\frac{0.2506}{5.009 * 0.1736}=0.2881 \\
& \sigma_{C_{D}} \approx|0.2881| \sqrt{\left(\frac{0.0160}{0.2847}\right)^{2}+\left(\frac{5.132 * 10^{-5}}{-0.0029-0.0400}\right)^{2}+\left(\frac{6.075 * 10^{-5}}{-0.0029-0.0400}\right)^{2}} \\
& \sigma_{C_{\boldsymbol{D}}} \approx \mathbf{0 . 0 1 6 2}
\end{aligned}
$$




\section{B. Sample Pressure Code}

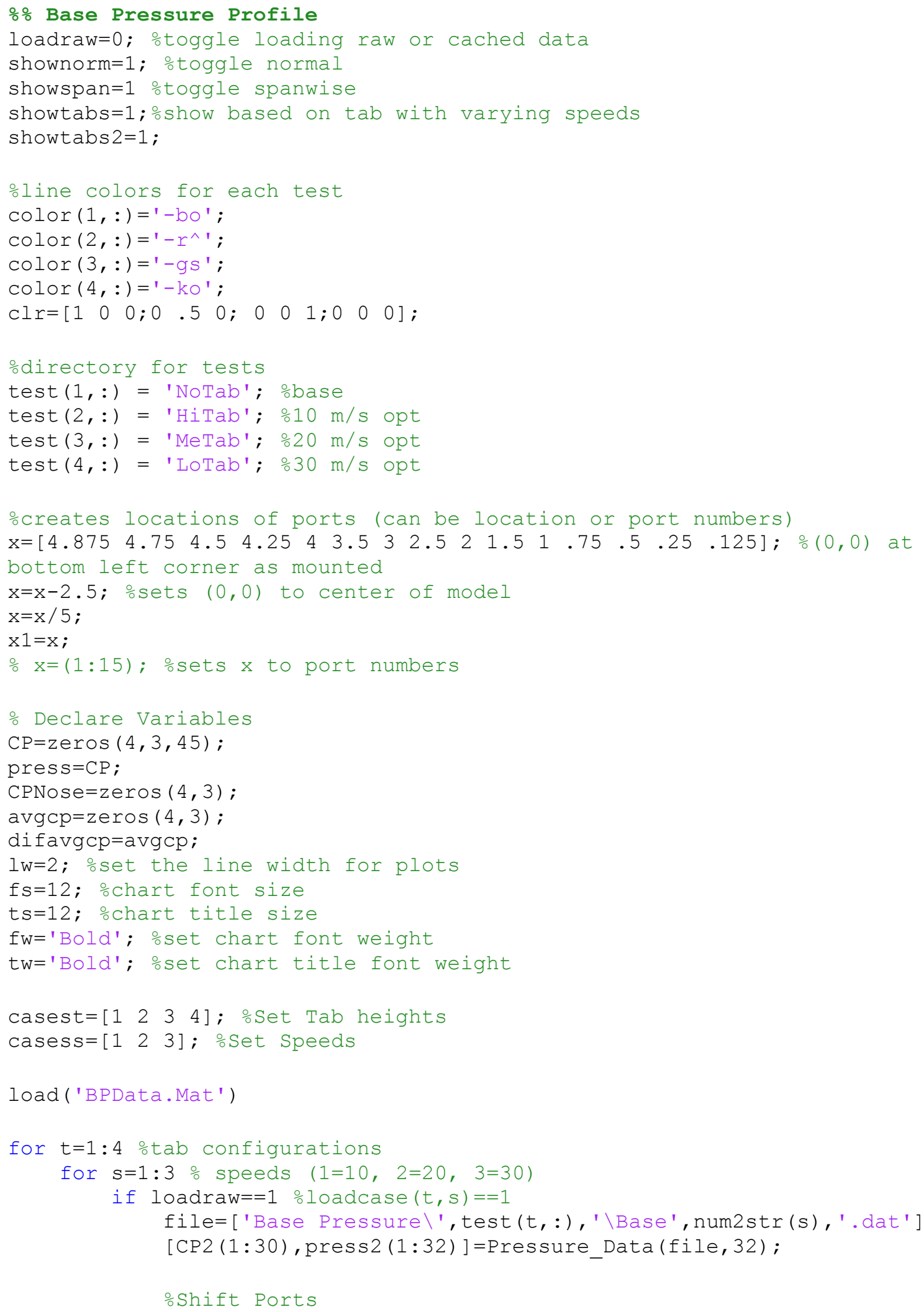




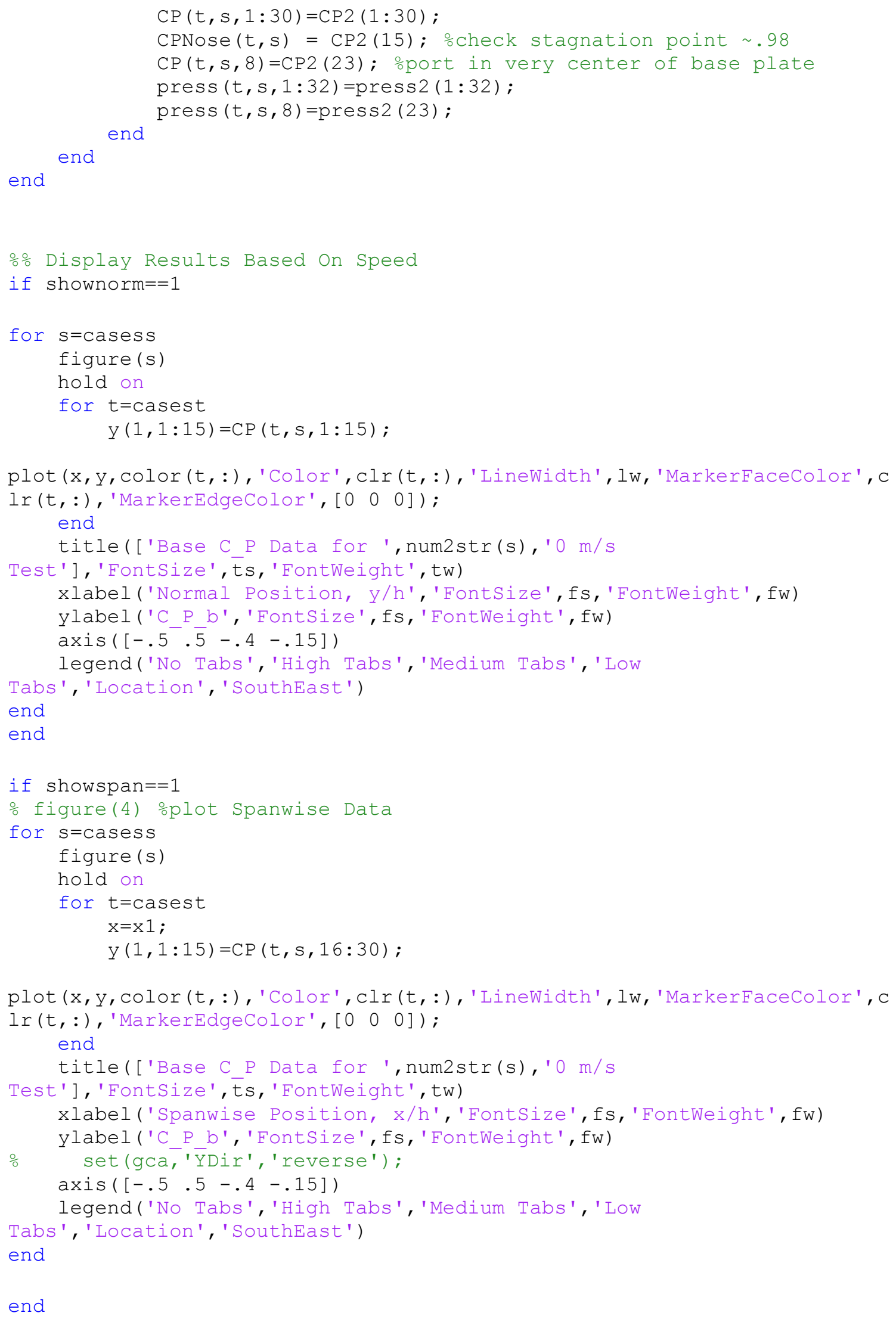




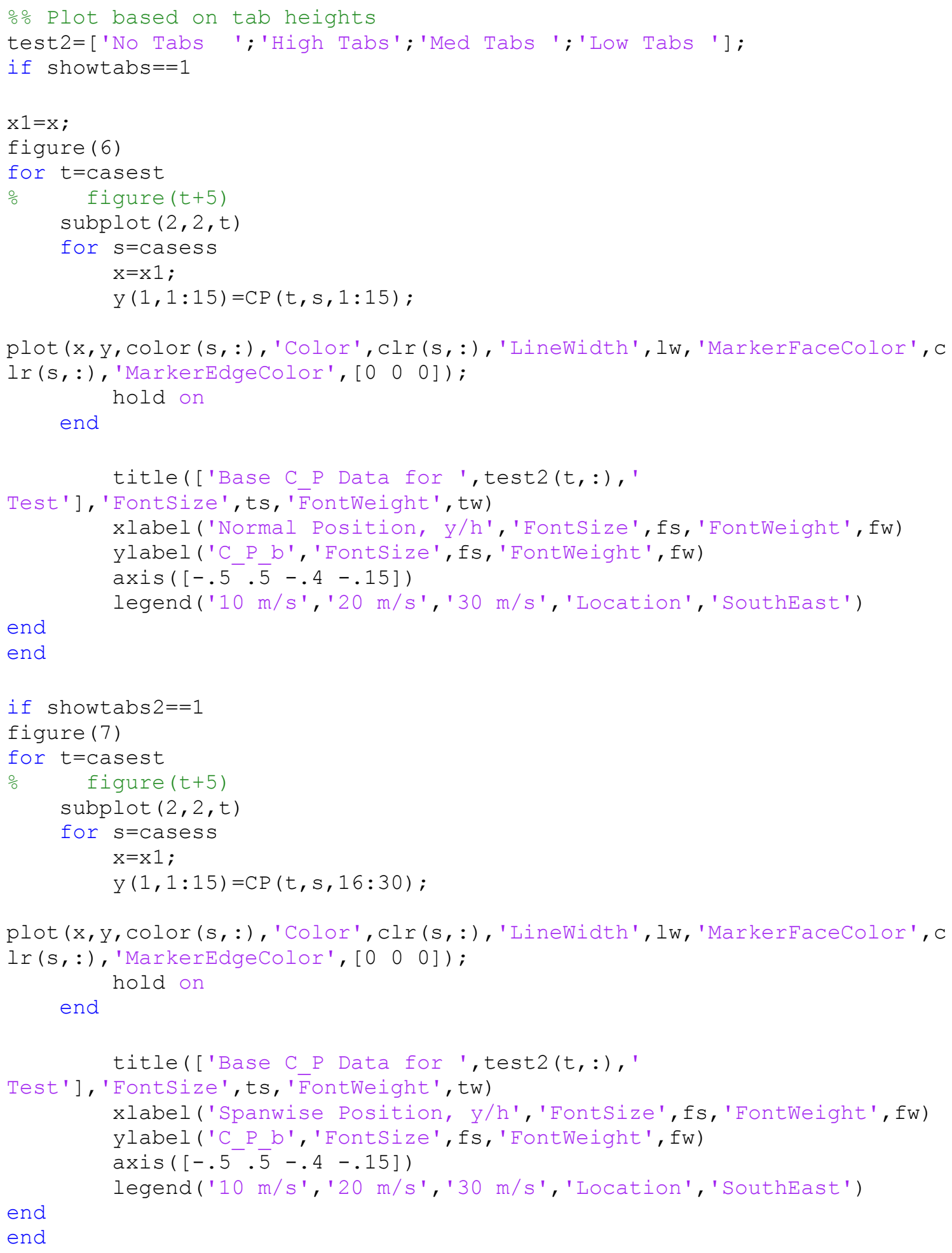

\section{Sample Boundary Layer Code}

clc; clear all;close all; format compact

fs=12; ochart font size 


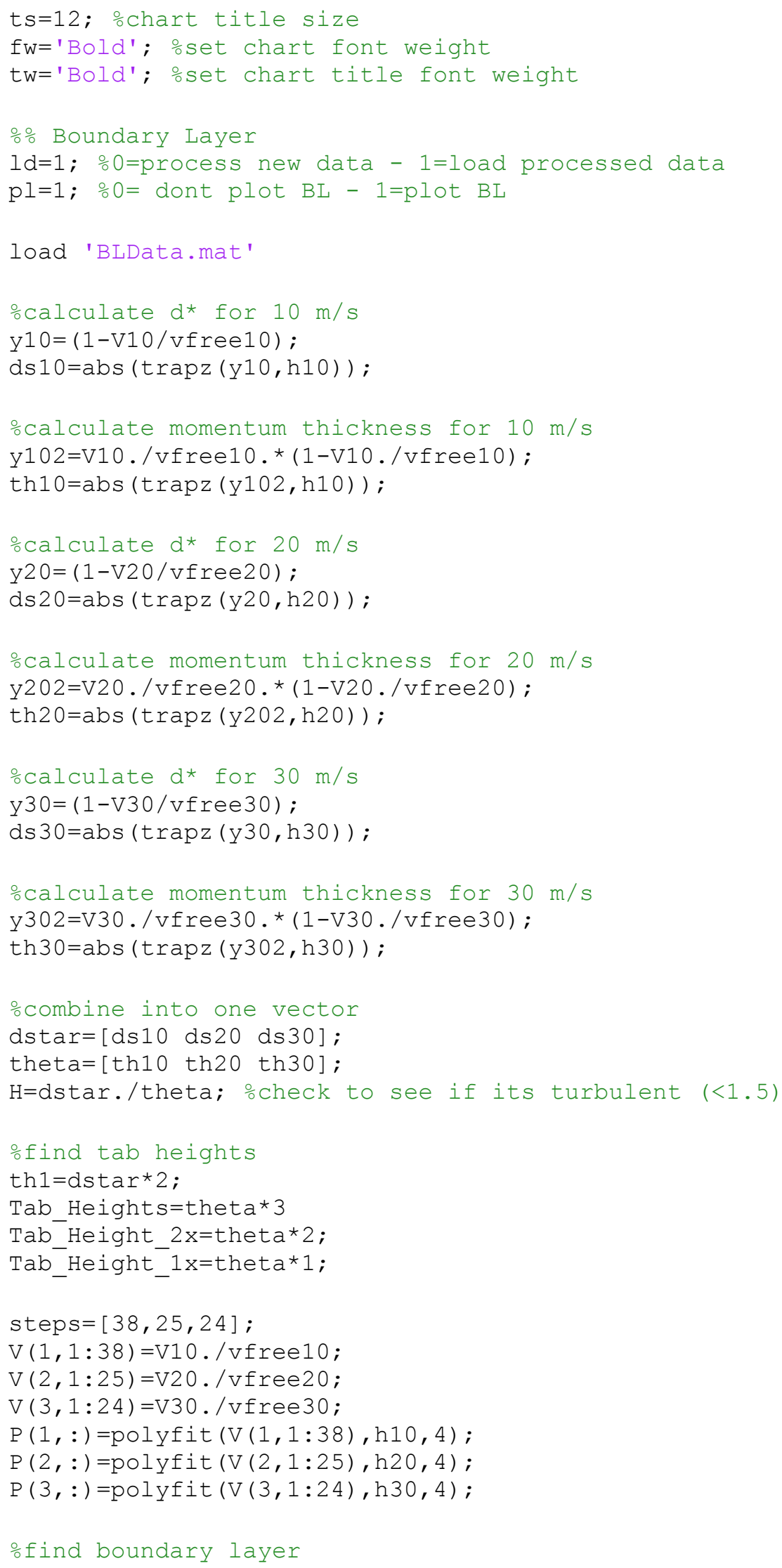




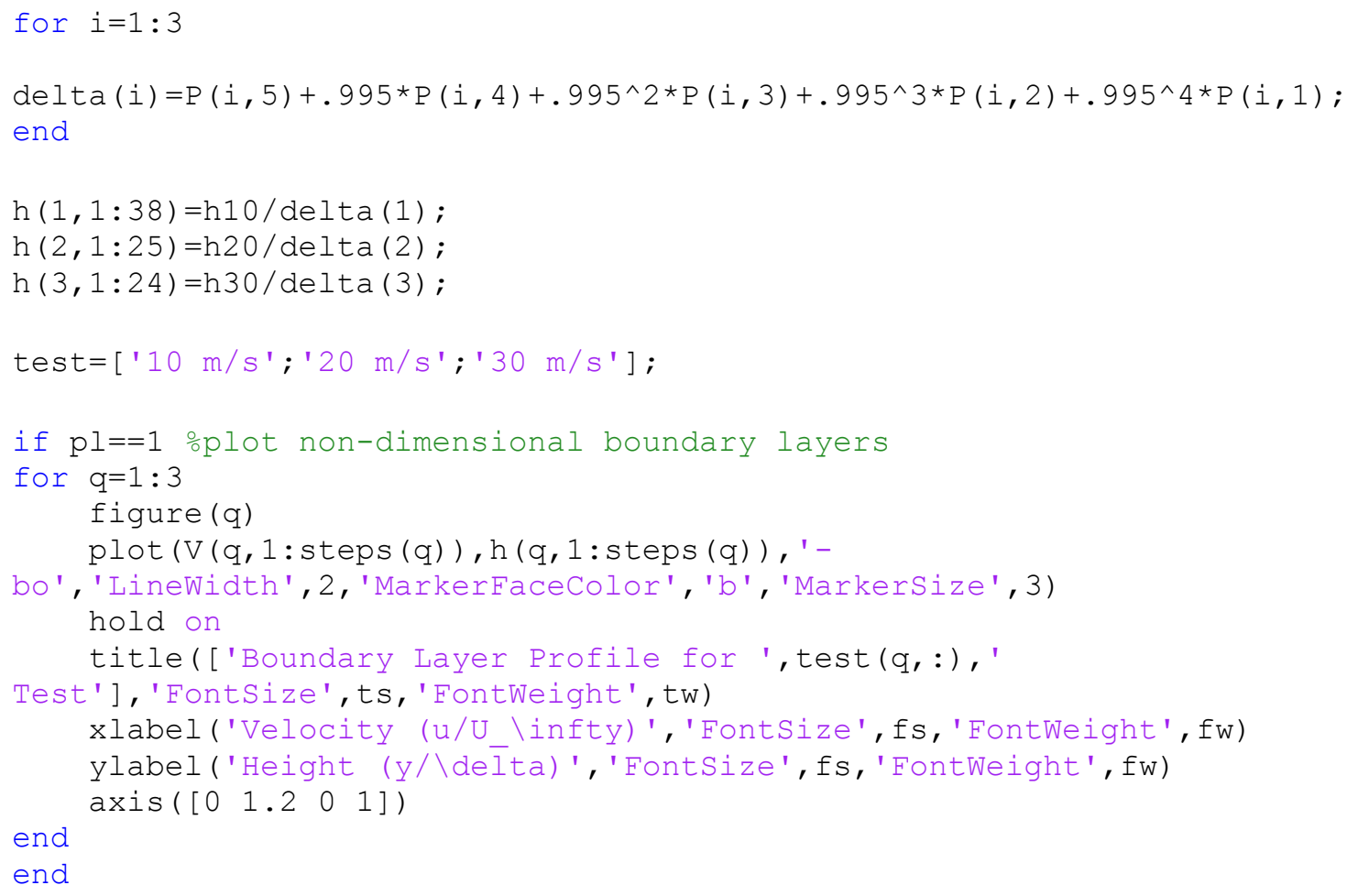

